\title{
FAMILY ACCOMMODATION IN HOARDING
}

by

\author{
Valerie Vorstenbosch \\ Master of Arts, Ryerson University, October 2009 \\ Bachelor of Science, Wilfrid Laurier University, June 2007
}

A dissertation
presented to Ryerson University

\author{
in partial fulfillment of the \\ requirements for the degree of \\ Doctorate of Philosophy \\ in the Program of \\ Psychology
}

Toronto, Ontario, Canada, 2013

(C) Valerie Vorstenbosch 2013 


\section{Author's Declaration for Electronic Submission of a Dissertation}

I hereby declare that I am the sole author of this dissertation. This is a true copy of the dissertation, including any required final revisions, as accepted by my examiners.

I authorize Ryerson University to lend this dissertation to other institutions or individuals for the purpose of scholarly research.

I further authorize Ryerson University to reproduce this dissertation by photocopying or by other means, in total or in part, at the request of other institutions or individuals for the purpose of scholarly research.

I understand that my dissertation may be made electronically available to the public. 


\title{
Family Accommodation in Hoarding \\ Doctorate of Philosophy 2013 \\ Valerie Vorstenbosch \\ Psychology Program \\ Ryerson University
}

\begin{abstract}
Hoarding, which includes the excessive acquisition of, and inability to discard, numerous possessions, is a debilitating mental health condition and is associated with significant family dysfunction and burden on family members. Currently, little is known about the effect that family members have on individuals' hoarding symptomatology and functioning, and vice versa. Thus, the present study examined the nature and frequency of family accommodation (i.e., the process by which family members participate in hoarding symptoms or modify personal and family routines in response to an individual's symptoms; Calvocoressi, Mazure, Stanislav, et al., 1999), in 52 individuals with self-reported hoarding problems and their close significant others (CSOs; i.e., intimate partner or family member). Participants completed the Family Accommodation Interview for Hoarding (FAI-H), which is an 11-item clinician-rated interview that was adapted from a previously validated measure for this study, and a series of self-report questionnaires. The FAI-H was found to be a valid and reliable assessment of accommodation in this hoarding sample. Most CSOs reported engaging in at least some accommodating behaviours; however, CSOs who lived with the individual with the hoarding problem engaged in accommodating behaviours more frequently than those who did not live with the individual with the hoarding problem. More than half of the CSOs endorsed hoarding participant-driven, as well as personally-driven motivations for engaging in accommodating behaviours, and believed that their accommodating behaviours were reasonable or helpful for both the individual with the
\end{abstract}


hoarding problem and themselves. Family accommodation was positively associated with hoarding symptom severity, relationship conflict, CSOs' rejecting attitudes toward the individual with hoarding problems, relationship problems, impairment in activities of daily living, and hoarding participant-rated anger. Family accommodation partially mediated the association between hoarding symptom severity and relationship conflict, averaging across hoarding participants and CSOs, and between hoarding symptom severity and impairment in activities of daily living for individuals with hoarding problems, but not CSOs. Results of the present study further elucidate the role of accommodation in hoarding, and increase our understanding of the interpersonal processes that may play an important role in problematic hoarding. 


\section{Acknowledgements}

First and foremost, I would like to thank my supervisor, Dr. Martin Antony, for his continuing support and guidance throughout my graduate training. He has provided me with ample opportunities and his commitment to my development as a scientist practitioner has prepared me well for a career as a clinical psychologist. I would like to thank my supervisory committee members, Dr. Candice Monson and Dr. Karen Rowa, whose support and guidance have helped to strengthen this project. I greatly appreciate their timely feedback and willingness to contribute to my growth as a researcher. In addition, I would also like to thank the additional members of my examining committee, Dr. Stephanie Cassin, Dr. Daria Romaniuk, and Dr. Gail Steketee, for agreeing to take time out of their busy schedules to review my dissertation and participate in my defense. I would like to thank Kevin Acuna for volunteering his time to help with recruitment, data entry, and fidelity ratings; the completion of this project would not have been possible without his help. I would like to thank my supportive labmates for the good times that we have had while working together, and my classmates for their helpful advice and support, their cooperative and friendly nature, and great memories, which will last a lifetime. Finally, I would like to thank all of my family and friends; without your love, encouragement, and patience, none of this would have been possible. 


\section{Dedication}

To my mom and dad 


\section{Table of Contents}

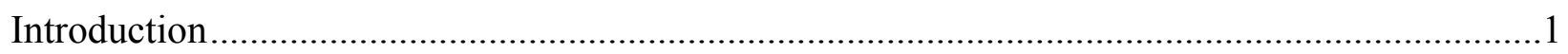

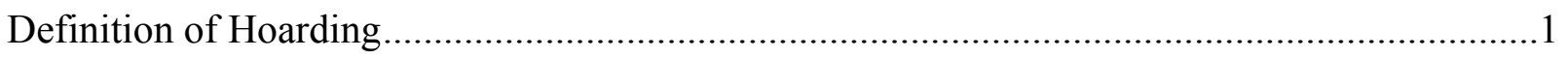

Differences between Hoarding and Obsessive-Compulsive Disorder .................................2

Excessive Acquisition ...........................................................................................

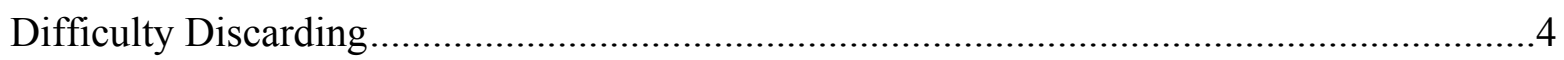

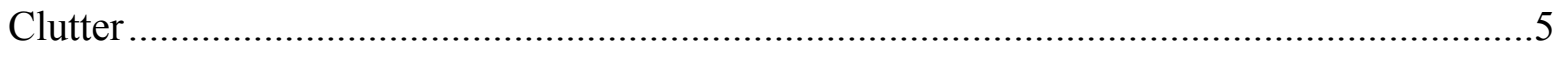

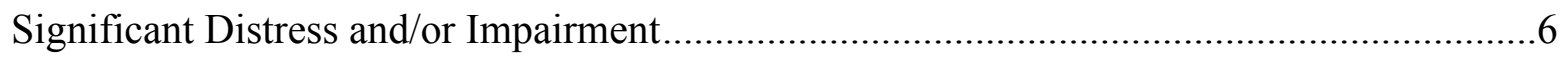

Cognitive-Behavioural Model of Hoarding ................................................................... 7

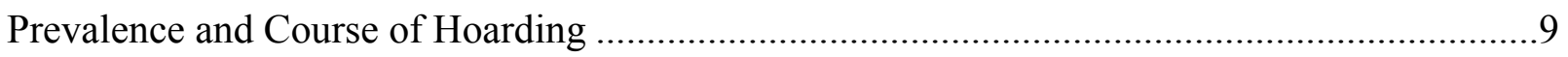

Effect of Hoarding Symptoms on Family Members .......................................................... 10

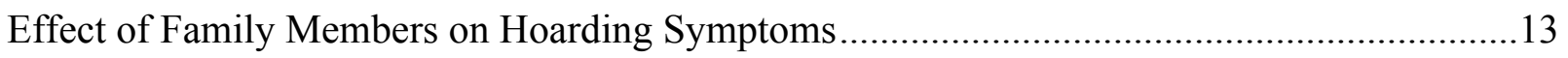

Family Accommodation in Obsessive-Compulsive Disorder .......................................14

Family Accommodation in Hoarding ..................................................................21

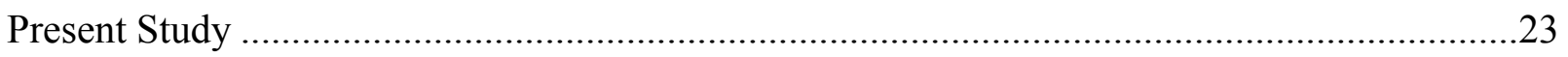

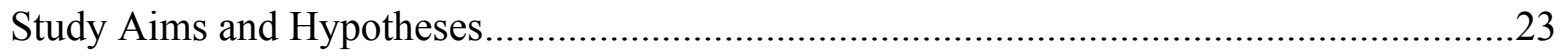

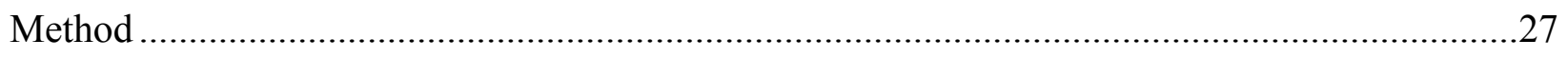

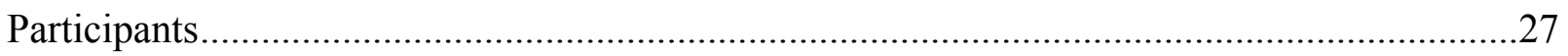

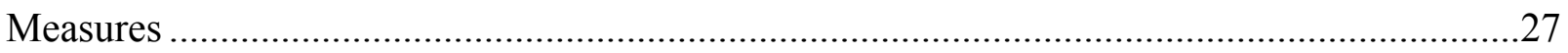

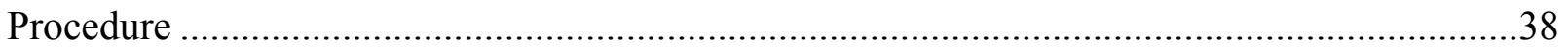

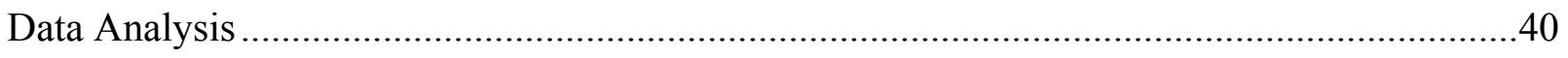

Actor-Partner Interdependence Model ............................................................... 43

Actor-Partner Interdependence Mediation Model......................................................45 
Results

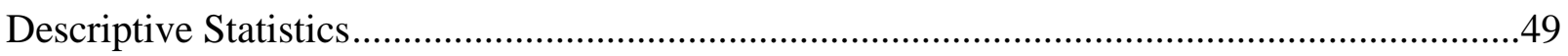

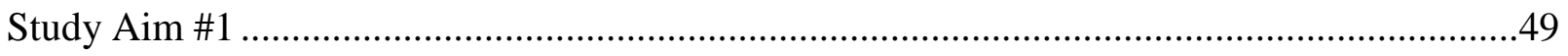

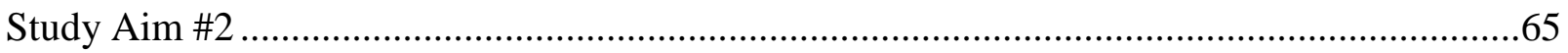

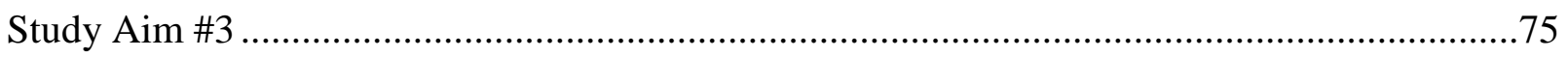

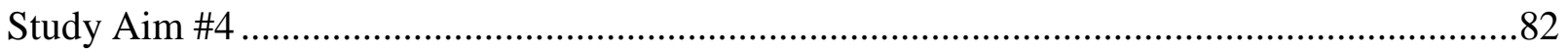

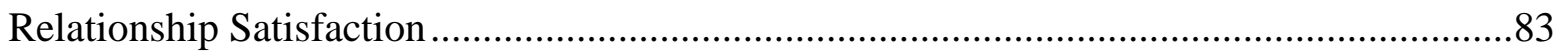

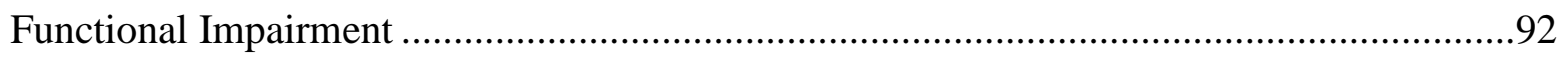

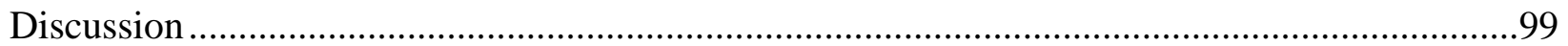

Psychometric Properties of the Family Accommodation Interview for Hoarding ...................99

Frequency and Nature of Family Accommodation in Hoarding .......................................102

Close Significant Others' Motivations for Engaging in Accommodating Behaviours............106

Close Significant Others' Attitude about Accommodating Behaviours ...............................110

Association between Family Accommodation and Hoarding Symptom Severity..................111

Association between Family Accommodation and Relationship Satisfaction ......................114

Association between Family Accommodation and Functional Impairment.........................117

Association between Family Accommodation and Wellbeing ..........................................118

Family Accommodation as a Potential Mediator of the Association between Hoarding

Symptom Severity and Relationship Satisfaction, as well as Impairment in Activities of

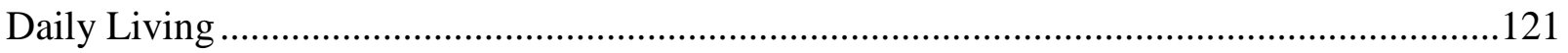

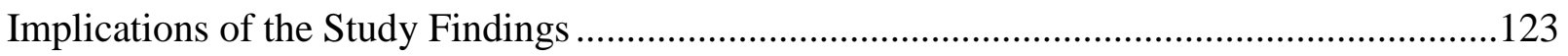

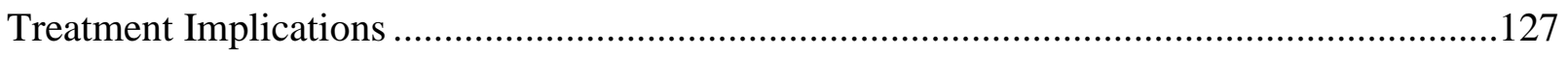

Strengths and Limitations of the Present Study .........................................................131 


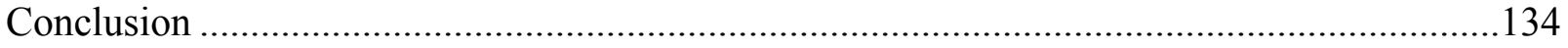

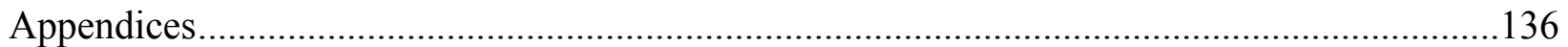

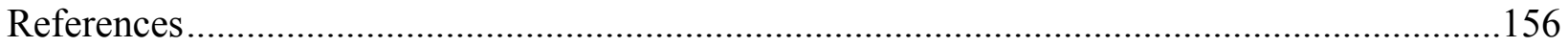




\section{List of Tables}

Table 1: Demographic Characteristics by Participant Status...................................................50

Table 2: Descriptive Statistics by Participant Status ..........................................................53

Table 3: Characteristics of the Family Accommodation Interview for Hoarding .......................58

Table 4: Convergent Validity of the Family Accommodation Interview for Hoarding ...............63

Table 5: Discriminant Validity of the Family Accommodation Interview for Hoarding .............64

Table 6: Means and Standard Deviations for Distress and Interference Associated with

Accommodating Behaviours that were Endorsed at Least Once or Twice per Month by

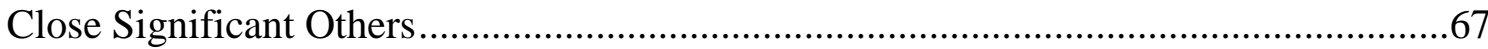

Table 7: Mann-Whitney U Tests Comparing Family Accommodation Scores for Intimate

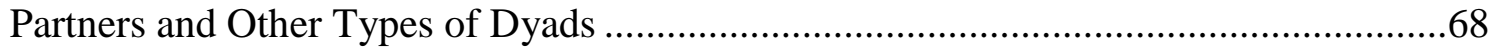

Table 8: Mann-Whitney U Tests Comparing Family Accommodation Scores for Close

Significant Others who Live with the Hoarding Participant and Close

Significant Others who do not Live with the Hoarding Participant..............................70

Table 9: Association between Family Accommodation and Close Significant Others'

Hoarding and Obsessive-Compulsive Disorder Symptoms.....................................

Table 10: Means and Standard Deviations for the Extent to Which Close Significant Others

Endorsed Each Motivation or Attitude, and the Number and Percentage of Participants

who Endorsed the Motivation or Attitude to at Least a Moderate Degree .....................72

Table 11: Association between Family Accommodation and Hoarding Participant-Driven and

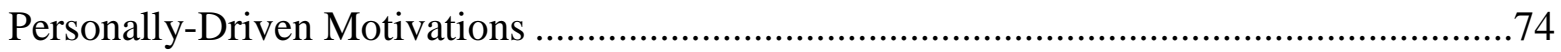

Table 12: Association between Family Accommodation and Hoarding Symptom Severity ........76

Table 13: Association between Family Accommodation and Relationship Satisfaction .............78 
Table 14: Association between Family Accommodation and Functional Impairment

Table 15: Association between Family Accommodation and Wellbeing. .81

Table 16: Interaction Model Estimating Effects of Overall Hoarding Symptom Severity on Relationship Support. .84

Table 17: Interaction Model Estimating Effects of Overall Hoarding Symptom Severity on Relationship Conflict .86

Table 18: Sobel Test Assessing Total Family Accommodation as a Mediator of the Association between Overall Hoarding Symptom Severity and Relationship Conflict .89

Table 19: Interaction Model Estimating Effects of Overall Hoarding Symptom Severity on Relationship Depth

Table 20: Interaction Model Estimating Effects of Overall Hoarding Symptom Severity on Activities of Daily Living

Table 21: Two-Intercept Model Estimating Effects of Overall Hoarding Symptom Severity on Activities of Daily Living for Hoarding Participants and Close Significant

Others.

Table 22: Sobel Test Assessing Total Family Accommodation as a Mediator of the Association between Overall Hoarding Symptom Severity and Activities of Daily Living for Hoarding Participants and Close Significant Others .98 


\section{List of Figures}

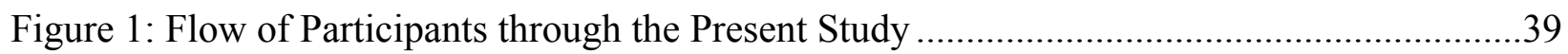

Figure 2: Actor-Partner Interdependence Model ..............................................................44

Figure 3: Actor-Partner Interdependence Mediation Model......................................................46

Figure 4: Actor-Partner Interdependence Mediation Model Assessing Total Family

Accommodation as a Mediator of the Association between Overall Hoarding Symptom

Severity and Relationship Conflict, Averaging Across Dyads ..................................8

Figure 5: Actor-Partner Interdependence Mediation Model Assessing Total Family

Accommodation as a Mediator of the Association between Overall Hoarding Symptom

Severity and Activities of Daily Living in Hoarding for Hoarding Participants and

Close Significant Others ................................................................................ 96 


\section{List of Appendices}

Appendix A: Family Accommodation Interview for Hoarding.............................................136

Appendix B: Interview Assessing Motivations for, and Attitudes About, Engaging in

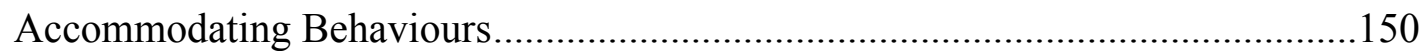

Appendix C: Hoarding Attribution Questionnaire............................................................. 155 


\section{Family Accommodation in Hoarding}

Hoarding is a common and debilitating mental health condition, with an estimated lifetime prevalence of between 2 and 5\% in the general population (Samuels et al., 2008). A recent study by the City of San Francisco estimated that problematic hoarding behaviours and clutter costs service providers and landlords in San Francisco approximately \$6.43 million dollars per year (San Francisco Task Force on Compulsive Hoarding, 2009). Previous research has documented a significant association between hoarding and relationship problems, including lower rates of marriage and higher rates of divorce for individuals with hoarding problems (Steketee \& Frost, 2003). In addition, family members have reported experiencing burden in response to living with or caring for a loved one with hoarding problems (Tolin, Frost, Steketee, \& Fitch, 2008). However, less is known about the effects that close significant others' (CSOs') behavioural responses have on individuals' hoarding symptoms, functional impairment, and wellbeing. Thus, the present study investigated the nature of CSOs' accommodating behaviours in a sample of individuals with self-reported hoarding problems and their CSOs, and explored the extent to which these behaviours were associated with hoarding symptom severity, relationship satisfaction, functional impairment, and wellbeing.

\section{Definition of Hoarding}

Hoarding has historically been considered a symptom or subtype of obsessive-compulsive disorder (OCD); however, accumulating research suggests that it is likely a distinct disorder (see Grisham, Brown, Liverant, \& Campbell-Sills, 2005, Pertusa et al., 2010, and Rachman, Elliott, Shafran, \& Radomsky, 2009 for reviews). As a result, several researchers have proposed diagnostic criteria for a new condition called "hoarding disorder" (Mataix-Cols et al., 2010), which was recently accepted for inclusion in the $5^{\text {th }}$ edition of the Diagnostic and Statistical Manual of Mental Disorders (DSM-5; American Psychiatric Association, 2013). 
Differences between hoarding and obsessive-compulsive disorder (OCD). Although phenomenologically an individual's fear about losing important items or documents could be conceptualized as an obsessional thought and an individual's avoidance or urge to save an item could be conceptualized as a compulsive behaviour, there are many important differences that have been identified between hoarding and OCD, which support hoarding disorder as a distinct disorder (Pertusa et al., 2010). Below is a summary of some of the evidence that Pertusa et al. (2010) provided in their recent review. First, the prevalence rate of problematic hoarding is about 5\% (Samuels et al., 2008), which is more than two times the rate of OCD, and only a minority of individuals with problematic hoarding report clinically significant OCD symptoms (e.g., only $17 \%$ of individuals with hoarding problems reported OCD symptoms in a community sample; Frost, Steketee, Tolin, \& Brown, 2006). In addition, other disorders (e.g., depression, social anxiety disorder, and GAD) have been found to be more comorbid with problematic hoarding than was OCD in this community sample and when OCD and hoarding were comorbid, the hoarding symptoms were unrelated to traditional OCD concerns (Frost et al., 2006). Second, hoarding symptoms are typically ego-syntonic (i.e., individuals with hoarding problems do not experience ego-dystonic intrusive thoughts, which lead them to perform a compulsive ritual; Pertusa et al., 2008), and individuals with hoarding problems tend to deny that they have a problem because they have poor insight regarding the severity of their problem; this contrasts with OCD symptoms, which are typically ego-dystonic, and associated with greater insight regarding the severity of their problem (Steketee \& Frost, 2003). Furthermore, the emotional experience of hoarding has been found to differ from that of OCD, such that individuals with hoarding problems tend to experience distress only when they are faced with having to discard an item, and when this is the case, they are more likely to experience grief, guilt, or anger, than 
anxiety (Pertusa et al., 2010). Finally, a review of pharmacological and psychological treatments has found that individuals with hoarding problems have a poorer treatment response to standard psychological treatment (e.g., exposure with response prevention) and medication for OCD (e.g., selective serotonin reuptake inhibitors), when compared with individuals who have nonhoarding OCD (Pertusa et al., 2010). Taken together, these results suggest that hoarding disorder may be better conceptualized as a distinct disorder, rather than a symptom or subtype of OCD (Pertusa et al., 2010).

In 1996, Frost and Hartl developed the following definition of problematic hoarding: "(1) the acquisition of, and inability to discard, a large number of possessions that appear to be useless or of limited value; (2) living spaces sufficiently cluttered so as to preclude activities for which those spaces were designed; and (3) significant distress or impairment in functioning caused by the hoarding" (p. 341). The hoarding disorder diagnosis that is included in DSM-5 builds on Frost and Hartl's (1996) original definition and includes the following six diagnostic criteria: (A) the person experiences persistent difficulty discarding personal possessions; (B) the difficulty discarding is due to distress, indecision, or a strong urge to save; (C) the symptoms result in clutter that prevents the normal use of the person's living space; (D) the symptoms cause clinically significant distress and/or impairment; (E) the symptoms are not due to the effects of a general medical condition; and (F) the symptoms are not better accounted for by symptoms of another mental disorder (American Psychiatric Association, 2013). In addition, there are also two specifiers included as part of the hoarding disorder diagnosis: (1) with excessive acquisition and (2) with good or fair insight, with poor insight, or with absent insight/delusional beliefs (American Psychiatric Association, 2013). 
Excessive acquisition. Many individuals with hoarding problems report spending excessive amounts of time acquiring possessions (Gilliam \& Tolin, 2010). In a recent study by Frost, Tolin, Steketee, Fitch, and Selbo-Bruns (2009), approximately $85 \%$ of individuals with self-reported hoarding problems reported difficulties with excessive acquiring. In addition, approximately $95 \%$ of family informants in this study reported that their family member had difficulties with excessive acquiring, which suggests that almost all individuals with hoarding problems have difficulties with acquiring.

Types of acquisition that have been observed among individuals with hoarding problems include compulsive buying, collecting free things, and stealing (Frost et al., 2009; Gilliam \& Tolin, 2010). For example, compulsive buying, which is defined as uncontrollable buying that is time consuming, causes significant distress, or results in social or financial difficulties (McElroy, Phillips, \& Keck, 1994), has been found to be associated with hoarding, such that individuals with hoarding problems have reported greater levels of compulsive buying when compared with healthy control participants (Frost et al., 1998). In addition, individuals with hoarding problems have also been found to report a greater tendency to acquire free items when compared with healthy control participants (Frost et al., 1998). Overall, the most frequent type of acquiring that is reported by individuals with hoarding problems is compulsive buying, followed by the excessive acquisition of free things and, more rarely, stealing (Frost et al., 2009).

Difficulty discarding. The failure to discard possessions is often described as the hallmark feature of hoarding (Frost \& Hartl, 1996). Some of the most common items that are saved by individuals with hoarding problems include clothes, books, souvenirs, greeting cards or letters, and bills or statements (Mogan, Kyrios, Schweitzer, Yap, \& Moulding, 2012). However, recent research also suggests that there may be an idiosyncratic nature to problematic hoarding, such 
that many individuals with problematic hoarding added extra items to the Savings List in the Mogan et al. (2012) study; examples of the various types of extra items that were added include videotapes, art materials, garden tools, dental floss, moisturizers and bubble baths from hotels, children's school work, children's toys, broken items, plastic bags, and recyclables (Mogan et al., 2012).

Research has suggested that individuals who hoard have difficulty with discarding because they believe that their possessions have sentimental, instrumental, or intrinsic value (Steketee, Frost, \& Kyrios, 2003). Sentimental value refers to the emotional attachment that a person feels with an object, while instrumental value refers to the beliefs that people have about the usefulness of an object (Furby, 1978). Intrinsic value, on the other hand, refers to the aesthetic appeal that a person believes an object has (Frost \& Rasmussen, 2012). Although many of these reasons for saving are similar to those provided by individuals who do not have hoarding problems (Frost \& Gross, 1993), research suggests that individuals with hoarding problems apply these reasons to save to a greater number and variety of possessions (Gilliam \& Tolin, 2010).

Clutter. As a result of problems with excessive acquiring and discarding, individuals' living spaces often become cluttered, so much so that they can no longer be used for their intended purpose (e.g., a person may not be able to sleep in his or her bed because of excessive clutter; Frost \& Hartl, 1996). In some cases, individuals' clutter may even extend beyond their home into vehicles, garages, storage lockers, etc. (Saxena \& Maidment, 2004). Clutter also tends to be disorganized, consisting of random piles of items that are of mixed importance (e.g., an individual's clutter may include a pile of candy wrappers, clothes, and important bills; Frost \& Rasmussen, 2012; Gilliam \& Tolin, 2010). Individuals with hoarding problems also tend to report a strong need to have their possessions in sight and poor organizational skills, which likely 
influences the level of clutter within their home. For example, individuals often report engaging in a process called "churning," which involves "moving items from one pile to another but never actually discarding any item nor establishing any consistent organizational system" (Saxena \& Maidment, 2004, p. 382).

Significant distress and/or impairment. Many individuals with hoarding problems report little distress or insight about the severity of their problem (Tolin, Fitch, Frost, \& Steketee, 2010); thus, a diagnosis of hoarding is more likely to be based on functional impairment, rather than self-reported distress (Tolin, Frost, Steketee, Gray, \& Fitch, 2008). Clutter, which often prevents the intended use of an individual's living space, tends to be associated with significant living impairment for individuals with hoarding problems and for those living within the home (Tolin, Frost, Steketee, \& Fitch, 2008). Specifically, cluttered living environments have been found to be associated with an increased risk of fire, falling, poor sanitation, and health problems (Steketee, Frost, \& Kim, 2001). Furthermore, in one research study, 8 to $12 \%$ of individuals with hoarding problems reported that they had been threatened with eviction or evicted because of their hoarding problem, and 0.1 to $3.0 \%$ indicated that they have had a child, elder, or pet removed from their home because of their hoarding problem (Tolin, Frost, Steketee, Gray, et al., 2008).

With regard to work impairment, individuals with hoarding problems have reported that much of their workspace is taken up by clutter, thereby making it difficult for them to find items (Tolin, Frost, Steketee, Gray, et al., 2008). On average, individuals with hoarding problems report taking 7.0 psychiatric impairment days per month, which is comparable to individuals with bipolar disorder or psychosis (Tolin, Frost, Steketee, Gray, et al., 2008). 


\section{Cognitive-Behavioural Model of Hoarding}

The cognitive-behavioural model of hoarding conceptualizes hoarding as a multifaceted problem that stems from personal vulnerability factors, such as childhood experiences, core beliefs (e.g., being unworthy, unlovable, or helpless), and deficits in information-processing, which are proposed to contribute to problems with emotional attachment to possessions and maladaptive beliefs about possessions, which in turn are proposed to create emotional responses that trigger hoarding-related behaviours (i.e., acquiring, saving/difficulty with discarding, and clutter); this model has been reviewed in numerous articles and book chapters, including Frost and Hartl (1996), Frost and Steketee (1998), Frost, Steketee, and Greene (2003), and Steketee and Frost (2007). Below is a summary of the model that is based on information provided in these sources. This initial CBT model is based on research that was completed with nonclinical hoarding samples, interviews that were completed with clinical hoarding samples, and attempts to change problematic hoarding behaviours; it is considered a work in progress, as many of the components still require empirical testing (Frost \& Hartl, 1996; Frost \& Steketee, 1998; Frost et al., 2003; Steketee \& Frost, 2007).

There are three types of information processing deficits that have been proposed to play a role in the development and maintenance of hoarding symptoms, including decision-making deficits, categorization and organization difficulties, and memory problems (Frost \& Hartl, 1996; Frost \& Steketee, 1998; Frost et al., 2003). Indecisiveness has been found among both clinical and nonclinical hoarding samples, and previous research has suggested that hoarding may be an avoidance behaviour that is related to indecisiveness and perfectionism (Frost \& Gross, 1993). In addition, individuals with hoarding problems display significant difficulties with categorization and organization, such that they tend to provide narrow definitions of categories so that relatively 
few items are able to fit within each category, and they tend to mix important possessions (e.g., paycheck) with unimportant possessions (e.g., gum wrapper) because they have trouble determining which possessions are important. In terms of memory problems, individuals with hoarding problems have been found to lack confidence in their memory and overestimate the importance of remembering information (Frost \& Hartl, 1996; Frost \& Steketee, 1998; Frost et al., 2003).

These deficits in information processing, in turn, are proposed to contribute to problems with emotional attachment to possessions and maladaptive beliefs about possessions. In the cognitive-behavioural model, individuals are proposed to save possessions for both sentimental and nonsentimental (e.g., instrumental or intrinsic) reasons, and past research has suggested that there are two types of sentimental attachment that may be associated with problematic hoarding. The first is pure sentimental attachment, which occurs when possessions serve as reminders of past events and are viewed as meaningful extensions of oneself. The second is a more securitybased form of attachment, which occurs when possessions act as a safety signal and are viewed as a source of comfort and security. In addition to emotional attachment problems, individuals are also proposed to experience maladaptive beliefs about possessions, including beliefs about vulnerability (e.g., individuals with hoarding problems believe that their possessions provide them with emotional comfort, and thus they believe that they are vulnerable without them), responsibility (e.g., individuals with hoarding problems believe that they have a responsibility to be prepared for the future and they believe that they are responsible for the proper care and use of possessions), memory (e.g., individuals with hoarding problems believe that their memories will be lost if they discard an item), and control (e.g., individuals with hoarding problems believe 
that they have to maintain control over their possessions; Frost \& Hartl, 1996; Frost \& Steketee, 1998; Frost et al., 2003; Steketee \& Frost, 2007).

The emotional responses that result from these problems with emotional attachments to possessions and maladaptive beliefs about possessions can be either positive or negative. The problems with emotional attachment are proposed to be associated with positive emotions, such as pleasure and pride, while maladaptive beliefs about possessions are proposed to be associated with negative emotions, such as sadness, anxiety, and guilt. These emotional responses are then proposed to trigger various hoarding-related behaviours (i.e., difficulties with discarding, excessive acquiring, and clutter). The cognitive-behavioural model of hoarding suggests that hoarding-related behaviours are maintained through positive reinforcement (i.e., through the pleasure that is gained from acquiring) or negative reinforcement (i.e., through the avoidance of negative emotions), and behavioural avoidance is conceptualized as being a prominent feature of hoarding (Frost \& Hartl, 1996; Steketee \& Frost, 2007). According to this model, hoarding behaviours allow individuals to avoid (1) making decisions, (2) making potential mistakes, (3) losing possessions that have emotional value, (4) losing information, opportunities, or something that they perceive as being part of their life, and (5) emotional distress, and as the individual's hoarding problem grows, an additional avoidance problem develops, such that the number of their possessions becomes too overwhelming to deal with (Frost \& Hartl, 1996; Frost \& Steketee, 1998; Frost et al., 2003).

\section{Prevalence and Course of Hoarding}

The prevalence of problematic hoarding is estimated to be between 2 and $5 \%$ in the general population (Iervolino et al., 2009; Samuels et al., 2008). The majority of individuals with hoarding problems have reported a chronic course of illness, with an age of onset in childhood or 
adolescence (Grisham, Frost, Steketee, Kim, \& Hood, 2006; Tolin, Meunier, Frost, \& Steketee, 2010). For example, Tolin, Meunier, and colleagues (2010) reported a median age of onset between 11 to 15 years and the majority of individuals reported an age of onset before 20 years.

Few individuals with hoarding problems have been found to experience a remission of symptoms, with the majority of individuals reporting a worsening of symptoms over time (Grisham et al., 2006). Thus, it should not be surprising that prevalence rates for problematic hoarding have been found to increase with age; individuals who are 55 years of age or older (6.2\%) were found to be three times more likely than individuals who are between 34 to 44 years of age (2.3\%) to experience hoarding problems (Samuels et al., 2008). Furthermore, although the estimated prevalence rate for problematic hoarding was not found to differ with education level, living arrangement, or ethnicity, it was found to be inversely related to household income, such that individuals with a lower income $(<\$ 20,000)$ were five times more likely than individuals with a higher income (> \$49,999) to report hoarding problems (Samuels et al., 2008).

\section{Effect of Hoarding Symptoms on Family Members}

As previously mentioned, hoarding has been found to be associated with significant functional impairment in individuals with the problem. However, in addition to the negative effects that hoarding has been found to have on the individuals with the problem, research has suggested that it may also have a detrimental effect on their family members or vice versa. For example, Frost and Gross (1993) found that $63 \%$ of individuals with hoarding problems report that their family members view their hoarding-related behaviours as problematic or bothersome. Furthermore, one of the most frequently cited problems by individuals in this study was conflict with their spouse or family member about clutter, which suggests that hoarding may have a negative impact on individuals' relationship functioning (Frost \& Gross, 1993). Further support 
for an association between hoarding and poor relationship functioning was provided by Frost, Steketee, and Williams (2000), who found that individuals with hoarding problems may also experience social withdrawl and rely on others to complete their necessary household tasks (e.g., cooking, cleaning, paying bills).

In an empirical study conducted by Frost, Steketee, Williams, and Warren (2000), the relation between hoarding and impairment across various domains was assessed using the Sheehan Disability Scale (SDS; Sheehan, 1983). The SDS assesses perceived disability in the areas of work, social life/leisure activities, and family life/home responsibilities. Results of this study found that hoarding participants with OCD reported greater impairment in the areas of social life/leisure activities and family life/home responsibilities when compared with nonhoarding participants with OCD, individuals with other anxiety disorders, and healthy control participants (Frost, Steketee, Williams, \& Warren, 2000). Specifically, individuals with OCD hoarding problems reported, on average, moderate impairment in the area of family life/home responsibilities (6.4/10), whereas individuals with OCD or another anxiety disorder only reported mild impairment, on average (4.2/10 and 2.2/10, respectively).

In an attempt to better understand family impairment, Wilbram, Kellett, and Beail (2008) conducted a qualitative analysis of interviews from 10 family members of individuals with hoarding problems. Five interconnecting themes emerged from these interviews, including: 1) loss of normal family life; 2) need for understanding; 3) coping with the situation; 4) impact on relationships; and 5) marginalization (Wilbram et al., 2008). Many participants described a loss of normal family life, including reduced living space and social life because of their family member's hoarding problem. For example, one participant reported that "several rooms [in their home] were now completely inaccessible" (Wilbram et al., 2008, p. 63), and another participant 
stated that they "don't follow up friendships and let them really develop because [they] know [they] can't keep up [their] end of it by inviting them [to their home]" (Wilbram et al., 2008, p. 64). In addition, family members also reported a need for understanding, such that they often tried to find a meaningful explanation for the hoarding problem, in hope of facilitating change; unfortunately, individuals' lack of insight about the severity of their hoarding problem was too large of a barrier for the family members to overcome. As a result, family members tried to make numerous attempts to cope with the situation by implementing strategies to reduce clutter (e.g., negotiating or modeling behaviour). Many of these attempted strategies, however, were unsuccessful. Because of family members' inability to facilitate change and reduce clutter, relationships were impacted, such that family members reported feelings of anger and frustration, as well as interpersonal conflict with the individual with the hoarding problem. Finally, many family members also reported feeling physically, emotionally and socially marginalized. For example, participants reported feeling 1) physically marginalized because they were continually being forced to use a smaller portion of their home; 2) socially marginalized because friends and neighbours failed to understand the hoarding and tolerate it in their lives; and 3) emotional distance in their relationship with the individual with the hoarding problem. Overall, results of this study suggest that hoarding is a systemic problem that impacts not only the individual with the hoarding problem but also the wider family (Wilbram et al., 2008).

Extending research in this area, Tolin, Frost, Steketee, and Fitch (2008) found that living in a cluttered environment during childhood was associated with greater distress, including reduced happiness, greater difficulty making friends, reduced socializing within the home, more strained relationships with parents, and greater embarrassment about the condition of one's home. In addition, family members and friends also reported a high degree of rejecting or hostile attitudes 
toward the individual with hoarding problems, which were assessed with the Patient Rejection Scale (PRS; Kreisman, Simmens, \& Joy, 1979). Increased hoarding symptom severity, poorer insight, and increased clutter in the home during childhood significantly predicted family members' negative attitudes toward individuals with hoarding problems (Tolin, Frost, Steketee, $\&$ Fitch, 2008). When compared with other mental health problems, Tolin, Frost, Steketee, and Fitch (2008) found that family members' scores on the PRS $(M=20.48, S D=4.57)$ were shown to be greater than those obtained from family members of treatment-seeking OCD patients $(M=$ $16.81 ; S D=3.84 ;$ Amir, Freshman, \& Foa, 2000) and were shown to be comparable to those obtained from family members of an outpatient schizophrenia sample $(M=19.4, S D=5.5$; Heresco-Levy, Brom, \& Greenberg, 1992). Although the temporal association between hoarding and familial distress or impairment has yet to be determined, results of these studies suggest that hoarding is associated with significant distress and impairment among CSOs of individuals with hoarding problems.

\section{Effect of Family Members on Hoarding Symptoms}

Given the systemic effects that have been observed among individuals with hoarding problems and the complex interactions that likely occur between these individuals and their CSOs, it is important to understand ways in which CSOs contribute to symptom expression and family dysfunction. Currently, however, little research has investigated the effect of CSOs' behaviours on hoarding symptoms. One construct that has received little attention among family members of individuals with hoarding problems, but has been found to play an important role in OCD symptom expression, is family accommodation (see Lebowitz, Panza, Su, \& Bloch, 2012 for a review of previous research that has investigated the role of family accommodation in children and adults with OCD). Family accommodation is defined as the process by which 
family members participate in an individual's symptoms and modify their personal or family routine because of the individual's mental health symptoms (Calvocoressi et al., 1995).

Family accommodation in obsessive-compulsive disorder (OCD). Approximately $88 \%$ of caregivers of individuals with OCD have been found to report at least some level of accommodation, with $38.2 \%$ reporting at least moderate levels of accommodation (Calvocoressi et al., 1995). Examples of ways in which family members have been found to accommodate OCD symptoms include providing reassurance, participating in patients' rituals, decreasing patient responsibility, and modifying one's leisure activities or family routine (Calvocoressi et al., 1995). Theoretically, family accommodation is proposed to play a maintaining role in OCD by negatively reinforcing patients' symptoms and preventing the reduction of anxiety (Renshaw, Steketee, Rodrigues, \& Caska, 2010). For example, many accommodating behaviours provide short-term relief for individuals because they give individuals the opportunity to avoid an anxiety-provoking situation or seek reassurance about their obsessive fears, which prevents them from experiencing a natural reduction in anxiety.

A transactional model of OCD has been developed to describe the reciprocal relationship between individuals' OCD symptoms and family functioning, which includes accommodation (Renshaw et al., 2010). This model was initially proposed by Van Noppen and colleagues (e.g., Livingston-Van Noppen, Rasmussen, Eisen, \& McCartney, 1990) and adapted by Renshaw, Steketee, and Chambless (2005). This model proposes interdependent relationships between OCD symptoms, family accommodation, and criticism/hostility by CSOs. According to this model, OCD symptoms lead to both accommodation and criticism/hostility from CSOs. In turn, accommodation is proposed to exacerbate or maintain OCD symptoms by reinforcing the belief that compulsions are necessary to alleviate anxiety, while overt criticism/hostility by CSOs is 
proposed to exacerbate or maintain OCD symptoms by creating a more stressful living environment. In addition, criticism/hostility by CSOs is proposed to exacerbate or maintain accommodation by increasing feelings of guilt, and CSOs who engage in accommodating behaviours are proposed to respond in a more critical/hostile manner because they are frustrated by their perceived need to engage in the accommodating behaviours. Neither the patient nor CSO is proposed to be at fault for the development of these specific behaviours and given that these relationships are considered to be interdependent, interventions that target any aspect of the cycle have been proposed to be effective in breaking the cycle of behaviours (Renshaw et al., 2010).

Family accommodation is often assessed via the Family Accommodation Scale for OCD (FAS for OCD; Calvocoressi, Mazure, Stanislav, et al., 1999; Calvocoressi, Mazure, Van Noppen, \& Price, 1999). A pilot version of this clinician-administered interview was developed in 1995. The pilot version consisted of nine core items that assessed a family member's participation in symptom-related behaviours and modifications of functioning, and four items that assessed distress or impairment that is experienced by the family member and the individual with OCD when the family member does or does not accommodate the symptoms (Calvocoressi et al., 1995). Truncated items of this pilot version have been used in other studies as a 13-item self-report measure.

Calvocoressi, Mazure, Stanislav, et al. (1999) refined the FAS for OCD based on their results of the pilot study. The FAS for OCD, which is a 12-item clinician-administered interview that assesses accommodation over the preceding week, consists of the following two sections. The first section assesses family members' perceptions of patients' OCD symptoms using a detailed symptom list that was adapted from the Yale-Brown Obsessive-Compulsive Scale (YBOCS; Goodman, Price, Rasmussen, Mazure, Delgado, et al., 1989; Goodman, Price, 
Rasmussen, Mazure, Fleischmann, et al., 1989). Results of this section are used to help the clinician formulate relevant examples of potential accommodating behaviours, which are assessed in the second section. The second section consists of the nine core items that were included in the pilot FAS for OCD and three new items, which were frequently reported by family members in the 1995 study but were not adequately captured by the pilot measure. Items in section two are scored on a 5-point Likert-type scale (0 - 4) and a total family accommodation score is calculated by summing the 12 items in the second section (higher scores represent greater levels of accommodation). The FAS for OCD has been found to have good psychometric properties, including good internal consistency (Cronbach's alpha $=.82)$ and excellent interrater reliability (intraclass correlations for individual items ranged from .75 to .99; Calvocoressi, Mazure, Stanislav, et al., 1999). In addition, the FAS for OCD has also demonstrated strong convergent and discriminant validity, with total scores being associated with measures of family functioning and stress but not measures of stress related to caring for a family member with nonOCD related problems (i.e., cognitive impairment, physical limitation, and terminal illness; Calvocoressi, Mazure, Stanislav, et al., 1999). This clinician-rated interview is considered the gold standard for measuring accommodation (Pinto, Van Noppen, \& Calvocoressi, in press). Although researchers have typically conceptualized accommodation as a unitary construct, Albert and colleagues (2010) found that it might be better conceptualized as a multidimensional construct. Results of Albert et al.'s (2010) study found that the 13-item pilot version of the FAS for OCD consisted of the following three factors: participation, modification, and distress and consequences. Furthermore, given that FAS-total, FAS-modification, and FAS-distress and consequences were associated with OCD symptom severity and health-related quality of life, but FAS-participation was not, it was suggested that different aspects of family accommodation 
could be associated with different types of impairment (Albert et al., 2010). However, because this was the first study to investigate the factor structure of the FAS for OCD, more research is required to confirm the dimensionality of family accommodation.

A recent review of the interpersonal model of OCD has suggested that family members may engage in accommodating behaviours for various reasons (e.g., they may accommodate their loved ones' OCD symptoms in an effort to reduce the patient's distress/impairment or ritual engagement; Renshaw et al., 2010). Empirically, Calvocoressi, Mazure, Stanislav, et al. (1999) found that $72 \%$ of primary caregivers believed that their accommodating behaviours alleviated patient distress, whereas $76 \%$ indicated that they engaged in accommodating behaviours in an attempt to decrease the amount of time that patients spent completing rituals. In addition, caregivers' OCD tendencies were found to be positively associated with family accommodation (Van Noppen \& Steketee, 2009), and parental anxiety symptoms, as well as the severity of children's compulsions, oppositional behaviours, and frequency of washing symptoms were found to be significant predictors of parental accommodation in a sample of children with OCD (Flessner et al., 2011). Taken together, these results suggest that some caregivers may accommodate their loved one's OCD symptoms because of their own anxiety.

A further understanding of what might guide CSOs' accommodating behaviours can be found within Weiner's (1986) attribution theory (Van Noppen \& Steketee, 2009). For example, it has been suggested that CSOs' attributions regarding patients' symptoms influence their emotional and behavioural responses, which, in turn, influence patient's symptomatology (Barrowclough \& Hooley, 2003). Two attributions that have been investigated in relation to family accommodation include responsibility and illness (Renshaw, Chambless, \& Steketee, 2006; Van Noppen \& Steketee, 2009). Specifically, previous research has found that caregivers 
who perceive patients' OCD symptoms to be within their control (i.e., caregivers who make responsibility attributions) are more hostile and critical, when compared with caregivers who perceive patients' OCD symptoms to be a result of their illness (i.e., caregivers who make illness attributions; Renshaw et al., 2006). However, previous research has suggested that caregivers who make illness attributions may be too tolerant and accommodating of patients' symptoms, which may, as a result, maintain patients' OCD symptomatology (Van Noppen \& Steketee, 2009).

Despite family members' good intentions, accommodation has been found to be positively associated with OCD symptom severity and family dysfunction (see Lebowitz et al., 2012 for a review). For example, Calvocoressi, Mazure, Stanislav, et al. (1999) found that family accommodation, as assessed with the FAS for OCD, was positively associated with OCD symptom severity, as well as rejecting attitudes toward individuals with OCD, and negatively associated with global family functioning. Ramos-Cerqueira, Rodrigues Torres, Torresan, Maranhao Negreiros, and Nakano Vitorino (2008) documented a positive association between family accommodation, as assessed with the 13-item pilot version of the FAS for OCD, and OCD symptom severity in a Brazilian sample. Amir et al. (2000) investigated the association between family accommodation and OCD symptom severity, using the 13-item self-report questionnaire. The following four subscales were generated: 1) participation in patients' OCD symptom behaviours; 2) modifications of family members' routines; 3) distress caused by accommodating the patient; and 4) negative consequences of refraining from accommodating. Results of this study found that modifications of family members' routine were positively associated with OCD symptom severity; no other associations were significant. 
Similar results have been found in studies that investigated family accommodation in pediatric OCD samples (see Lebowitz et al., 2012 for a review). Like Amir et al. (2000), Peris et al. (2008) assessed family accommodation using the 13-item self-report questionnaire. They generated the following scores: 1) total accommodation (all 13 items), 2) modification of family routines (5 items), 3) participation in rituals (4 items), 4) distress associated with accommodating (1 item), 5) negative consequences of refraining from accommodating ( 3 items), and 6) total involvement (5 modification items and 4 participation items). Although child OCD symptom severity was not significantly associated with total family accommodation, it was shown to be positively associated with total involvement, participation in rituals, modification of family routines, and negative consequences of refraining from accommodating. In addition, family accommodation was also significantly associated with family functioning (i.e., family cohesion, conflict and organization). Specifically, family cohesion was negatively associated with parents' distress about accommodating, and negative consequences of refraining from accommodating. Family organization was negatively associated with total accommodation, modification of family routines, parents' distress about accommodating, and total involvement. Family conflict was positively associated with parents' distress about accommodating and negative consequences of refraining from accommodating. Consistent with the adult literature, Storch, Geffken, Merlo, Jacob, et al. (2007) found a positive association between family accommodation (assessed with the 13-item pilot version of the FAS for OCD) and OCD symptom severity, as well as parentrated child functional impairment in a sample of children and adolescents with OCD and their parents. Moreover, this study also found that family accommodation mediated the association between OCD symptom severity and parent-rated child functional impairment, and this finding was replicated in a recent study, which also found that family accommodation mediated the 
association between OCD symptom severity and parent-rated functional impairment (Caporino et al., 2012). Taken together, these results suggest that family accommodation may be one mechanism through which OCD symptom severity leads to greater functional impairment (Caporino et al., 2012; Storch, Geffken, Merlo, Jacob, et al., 2007). Although cross-sectional, results of these studies suggest that family members' accommodating behaviours have profound effects on adults and children with OCD.

Family accommodation has also been found to be negatively associated with the wellbeing of caregivers of individuals with OCD, as well as individuals with OCD (see Lebowitz et al., 2012 for a review). For example, Amir et al. (2000) found that family accommodation was positively associated with depression and anxiety among relatives of individuals with OCD. Specifically, using the 13-item self-report questionnaire for accommodation, these researchers found that self-reported depression was positively associated with modifications of family members' routines, distress caused by accommodating the patient, and the negative consequences of refraining from accommodating. Self-reported anxiety, however, was only shown to be positively associated with distress caused by accommodating the patient, and the negative consequences of refraining from accommodating. Peris et al. (2008) found that family accommodation was positively associated with parental psychopathology in a sample of children and adolescents with OCD and their parents. The global severity index of the Brief Symptom Inventory (BSI; Derogatis, 1993) was found to be positively associated with all accommodation scores, with the exception of parental distress. In addition, parental hostility was found to be positively associated with all accommodation scores, with the exception of participation in rituals, and parental anxiety was found to be positively associated with total involvement (Peris et al., 2008). Extending research in this area, Ramos-Cerqueira et al. (2008) found that (1) family 
accommodation was positively associated with caregiver's emotional burden, and (2) caregivers who reported at least moderate levels of perceived distress for caring (as assessed with item 10 of the pilot version of the FAS for OCD) experienced greater levels of emotional burden. Family accommodation has also been found to be negatively associated with comorbid symptoms (e.g., depression and anxiety) in adults and children with OCD. For example, family accommodation, as assessed with the 13-item pilot version of the FAS for OCD, was found to be associated with internalizing and externalizing symptoms in children with OCD (Caporino et al., 2012; Storch, Geffken, Merlo, Jacob, et al., 2007). In addition, Albert and colleagues (2010) found that the family members modifications and overall accommodation (as assessed with the 13-item pilot version of the FAS for OCD) were associated with patients' depression in a sample of adults with OCD and their family members. Once again, although the cross-sectional nature of these studies makes it difficult to determine the temporal association between family accommodation and wellbeing, results of these studies suggest that the accommodation of OCD symptoms may be negatively associated with the wellbeing of caregivers, as well as adults and children with OCD.

Family accommodation in hoarding. To date, two studies have investigated family accommodation in hoarding. A qualitative study by Wilbram and colleagues (2008) found that family accommodation frequently occurred among family members of individuals with hoarding problems. Specifically, family members reported that they accommodated their loved one's hoarding problem by tolerating the continued acquisition of clutter and "walking 'on eggshells' around the home, in fear of disturbing clutter with the resultant conflict and distress" (Wilbram et al., 2008, p. 70). A recent quantitative study by Steketee, Ayers, Umbach, Tolin, and Frost (2013) investigated the construct of family accommodation in a large Internet sample of family 
members of individuals with hoarding problems. Accommodation was assessed using the 16item self-report Family Response to Hoarding Scale (FRHS; Steketee et al., 2013), which was adapted from the FAS for OCD specifically for this study. The FRHS contains two subscales, including the family behaviour changes subscale, which assesses the extent to which family members change their behaviours toward the individual with the hoarding problem, and the family consequences subscale, which assesses the adverse impact that an individual's hoarding behaviours have on family members. Overall, total accommodation was found to be positively associated with hoarding symptom severity; however, the family consequences subscale was found to be more strongly associated with hoarding symptom severity than the family behaviour changes subscale. This finding is consistent with Albert et al. (2010) who found that FASmodification and FAS-distress and consequences were significantly associated with OCD symptom severity and health-related quality of life, whereas FAS-participation was not. Furthermore, in the Steketee et al. (2013) study, family members were also found to be more likely to change their behaviour towards the individual with the hoarding problem and experience adverse consequences because of their loved one's hoarding behaviours if they had a hoarding problem themselves, were adults, and were living with the individual with the hoarding problem during the past 10 years. Taken together, the results of these two studies suggest that accommodation is present among families of individuals with hoarding problems; however, no research to date has investigated the association between family accommodation and relationship satisfaction, functional impairment, or wellbeing in a sample of individuals with hoarding problems and their CSOs. 


\section{Present Study}

The purpose of the present study was to investigate the construct of family accommodation in hoarding, using a sample of individuals with self-reported hoarding problems and their CSOs. CSOs included partners, spouses, or family members of individuals with hoarding problems. The present study had four aims.

Study aims and hypotheses. The first aim of the study was to adapt the FAS for OCD (Calvocoressi, Mazure, Stanislav, et al., 1999; Calvocoressi, Mazure, Van Noppen, et al., 1999) specifically for hoarding and investigate its psychometric properties (i.e., its reliability and validity). The adapted measure has been entitled the Family Accommodation Interview for Hoarding (FAI-H), and the initial items and format of the FAI-H were adapted from the FAS for OCD with permission (L. Calvocoressi, personal communication, May 15, 2012).

Reliability is defined as "the consistency with which all of a scale's items measure the same construct, and the consistency with which the total scale measures that construct in the same way every time" (Myers \& Winters, 2002, p. 119). There are four types of reliability, including internal, test-retest, interrater, and parallel-forms. Internal consistency measures the extent to which individual items on a scale are consistent with each other and is measured using Cronbach's alpha (Myers \& Winters, 2002). Test-retest reliability measures the extent to which a scale is stable over time. Correlations greater than .80 represent adequate stability over a 1 - to 2 week period, and correlations greater than .70 represent reasonable stability over a 1-month period (Myers \& Winters, 2002). Interrater reliability refers to the agreement that exists between different informants and is measured using the intraclass correlation coefficient (Hogan, 2008). Correlations greater than .80 are classified as acceptable (Myers \& Winters, 2002). Parallelforms reliability measures agreement between two forms of a scale (e.g., long and short versions). 
Correlations greater than .80 are classified as adequate parallel-forms reliability (Myers \& Winters, 2002).

Validity is defined as the extent to which a "scale accurately assesses what it was designed to assess" (Myers \& Winters, 2002, p. 120). There are three main types of validity, including content, criterion, and construct. Content validity assesses the extent to which a scale's items are relevant to and represent the construct that is being measured (Haynes, Richard, \& Kubany, 1995). Criterion validity is measured in relation to other valid scales. There are two types of criterion validity: predictive (i.e., a scale's association with a future event) and concurrent (i.e., a scale's association with an event that is measured simultaneously; Myers \& Winters, 2002). In addition, there are two types of concurrent validity, including convergent (i.e., the extent to which a scale is associated with a scale that assesses a theoretically relevant construct) and discriminant (i.e., the extent to which a scale is poorly associated with a scale that assesses a theoretically distinct construct; Moretz \& McKay, 2008). Construct validity refers to the extent to which a scale measures a particular theoretical construct. A scale is considered to have strong construct validity if it is shown to have convergent and discriminant validity (Myers \& Winters, 2002).

The present study assessed the internal consistency, interrater reliability, convergent validity, and discriminant validity of the FAI-H. Test-retest reliability and predictive validity were not assessed because the present study was cross-sectional and not longitudinal. Given that the FAS for OCD has been shown to have strong psychometric properties, including good internal consistency and excellent interrater reliability (Calvocoressi, Mazure, Stanislav, et al., 1999), it was anticipated that the FAI-H would also yield strong psychometric properties. 
The second aim of the study was to explore the nature of family accommodation in hoarding by exploring the frequency and severity of family accommodation in a hoarding sample, as well as CSOs' motivations for, and attitudes about, engaging in accommodating behaviours. The association between family accommodation and CSOs' own hoarding and OCD symptoms were assessed to determine the extent to which these symptoms may have influenced CSOs' levels of accommodation. It was hypothesized that there would be positive associations between family accommodation and CSOs' hoarding symptoms, as well as OCD symptoms.

The frequency with which CSOs endorsed hoarding participant-driven, as well as personally-driven motivations for engaging in accommodating behaviours, and the frequency with which CSOs endorsed various attitudes about engaging in accommodating behaviours was investigated. Like OCD, it was expected that CSOs would report hoarding participant-driven, as well as personally-driven motivations for engaging in accommodating behaviours. It was also anticipated that there would be positive associations between family accommodation and hoarding participant-driven motivations for engaging in accommodating behaviours, as well as personally-driven motivations for engaging in accommodating behaviours.

The third aim of the study was to investigate the construct of family accommodation in hoarding by examining the association between CSOs' accommodating behaviours and: 1) hoarding participant- and CSO-rated hoarding symptom severity; 2) relationship satisfaction; 3) hoarding participant- and CSO-rated functional impairment; 4) CSOs' attribution of hoarding participants control over hoarding behaviours; 5) CSOs' rejecting attitudes toward hoarding participants, and 6) CSOs' and hoarding participants' wellbeing (i.e., depression, anxiety, stress, and anger). Consistent with previous research, it was hypothesized that CSOs' accommodating behaviours would be positively associated with hoarding symptom severity, functional 
impairment, and increased rejection toward the individual with hoarding problems. In addition, it was also hypothesized that CSO's accommodating behaviours would be negatively associated with relationship satisfaction, CSOs' attribution of hoarding participants control over their hoarding behaviour, and CSOs' and hoarding participants' wellbeing.

The fourth aim of the study was to investigate the extent to which family accommodation mediated the relation between hoarding symptom severity and relationship satisfaction, as well as the relation between hoarding symptom severity and functional impairment. Given that Storch, Geffken, Merlo, Jacob, et al. (2007) and Caporino et al. (2012) found that family accommodation mediated the association between OCD symptom severity and parent-rated functional impairment, it was expected that family accommodation would also mediate the relation between hoarding symptom severity and relationship satisfaction, as well as the relation between hoarding symptom severity and functional impairment. 


\section{Method}

\section{Participants}

Fifty-two dyads, consisting of one individual with self-reported hoarding problems and one CSO (i.e., intimate partner or family member), were recruited for the present study. Participants were recruited from the community using flyers and newspaper advertisements. To be eligible to participate, individuals had to 1) be between 18-75 years of age, 2) obtain a score above 14, which is the clinical cutoff for hoarding, on the self-report version of the Hoarding Rating ScaleSelf Report (HRS-SR; Tolin, Frost, Steketee, \& Gray, et al., 2008), and 3) have a CSO, with whom they spend an average of at least 4 hours per week, who was willing to participate and did not obtain a score above the clinical cutoff for hoarding on the HRS-SR. The last inclusion criterion was included so that the individual with the hoarding problem and CSO could be clearly differentiated based on their prescreen HRS-SR scores. All participants consented to have their telephone interviews audio-recorded. Individuals were excluded from the study if they presented with any condition that limited their ability to provide reliable data (e.g., current psychosis, inability to comprehend English). Participants received $\$ 40$ remuneration for participating in the study.

\section{Measures}

Mini International Neuropsychiatric Interview (MINI; Lecrubier et al., 1997). The MINI is a short, semistructured clinician-rated interview for DSM-IV that assesses current and lifetime Axis I disorders. The MINI was administered by a trained, supervised graduate student via telephone. The MINI was administered to the individual with hoarding problems, as well as his/her CSO, and was used to describe the sample. One research assistant, who was trained on the administration of the MINI by the author, coded a subset of interviews ( $n=10$ interviews) 
and kappa coefficients were computed for each diagnosis to assess interrater reliability. Interrater reliability for current and lifetime MINI diagnoses was excellent for all diagnoses $(\kappa=1.00)$, except lifetime depression, which was moderate $(\kappa=.62)$.

Family Accommodation Interview for Hoarding (FAI-H). The FAI-H is an 11-item clinician-rated interview that was developed for the present study (see Appendix A). The initial items and format were adapted from the FAS for OCD (Calvocoressi, Mazure, Stanislav, et al., 1999; Calvocoressi, Mazure, Van Noppen, et al., 1999) specifically for hoarding, with permission (L. Calvocoressi, personal communication, May 15, 2012). Like the FAS for OCD, the FAI-H consists of two sections. The first section asks CSOs to identify the hoarding participant's current hoarding symptoms, using the HRS-I (Tolin, Frost, \& Stektee, 2010). This is done to help the clinician provide examples of potential accommodating behaviours. The second section consists of 11 items that assess the extent to which CSOs participate in, or tolerate the hoarding participant's hoarding behaviours and modify their behaviours or routine because of the individual's hoarding behaviors. For this study, accommodating behaviours were assessed in two ways: First, participants were asked to rate the frequency with which they engaged in a specific accommodating behaviour during the past month and second, they were asked to rate the extent to which they were bothered by that accommodating behaviour or experienced interference in their life because of that accommodating behaviour during the past month. Frequency is rated on a 5 -point scale, ranging from $0=$ Never to $4=$ Daily, and distress is rated on a 5-point scale, ranging from $0=$ Not at all to $4=$ Extreme. The 11 frequency items are summed to yield a total frequency score and the 11 distress/interference items are summed to yield a total distress/interference score. A total score is generated by summing the frequency and distress/interference subscales. The psychometric properties of the FAI-H were assessed as part 
of the present study. CSOs and hoarding participants completed the FAI-H; CSOs reported on their own accommodating behaviours, whereas hoarding participants completed a modified version of the FAI-H that assessed their perceptions of their CSOs' accommodating behaviours.

Family Response to Hoarding Scale (FRHS; Steketee et al., 2013). The FRHS is a 16item self-report measure, which was adapted from the FAS for OCD (Calvocoressi, Mazure, Stanislav, et al., 1999; Calvocoressi, Mazure, Van Noppen, et al., 1999). It includes classic accommodation items from the FAS (e.g., waiting for the individual with the hoarding problem, engaging in odd/senseless behaviours, taking over responsibilities, and modifying personal or family routines), as well as additional items based on the developers' experiences working with individuals with hoarding problems. It assesses the extent to which a CSO alters his or her behaviours in response to living with an individual with hoarding problems or experiences problems because of an individual's hoarding symptoms. Participants are first asked to indicate whether they engaged in a specific behaviour or experienced a specific problem during the past month; if they endorse an item, they are then asked to rate the frequency or severity of that item using a scale, ranging from 1 (1-2 times/mild severity) to 4 (10 or more times/very severe). In addition to the total scale, the FRHS also includes two subscales: Family Behaviour Changes (items 1-7 and 11), which assess the extent to which CSOs change their behaviours toward the individual with the hoarding problem; and Family Consequences (items 12-16), which assesses the extent to which CSOs experience behavioural consequences or an adverse impact because of an individual's hoarding symptoms. Although Steketee et al. (2013) found that items 8-10 did not clearly load on either subscale, they were retained in the final 16-item scale because their retention did not degrade reliability. Total FRSH scores range from 0 to 64. Family Behaviour Changes scores range from 0 to 32 and Family Consequences scores range from 0 to 20. The 
FRSH has demonstrated good psychometric properties, including good internal consistency for the total score and the Family Behaviour Changes subscale score, and acceptable internal consistency for the Family Consequences subscale score (Steketee et al., 2013). The present study used CSO-rated FRSH total and subscale scores to assess the convergent validity of the FAI-H. Internal consistency was excellent for total FRHS scores $(\alpha=.92)$ and good for Family Behaviour Changes subscale scores and Family Consequences subscale scores $(\alpha=.86$ and $\alpha$ $=.82$, respectively) in the present study.

\section{Interview Assessing Motivations for, and Attitudes About, Engaging in}

Accommodating Behaviours. Following the completion of the FAI-H, CSOs were interviewed about their motivations for, and attitudes about, engaging in accommodating behaviours to assess potential reasons why they may have accommodated their loved one's hoarding symptoms (see

Appendix B). Following from Calvocoressi, Mazure, Stanislav, et al.'s (1999) study, CSOs were asked to rate their motivations for engaging in accommodating behaviours (i.e., they were asked to rate the extent to which they or the individual with the hoarding problem drive the accommodating behaviours). CSOs were asked to rate how much they believe each potential motivation was true on a 5-point scale, ranging from 0 (not at all) to 4 (extremely). Two motivation subscale scores were generated: a hoarding participant-driven score and a personallydriven (or CSO-driven) score. Five hoarding participant-driven and five personally-driven items were summed to yield the hoarding participant-driven and personally-driven subscale scores, respectively. In addition, CSOs were also asked to rate the extent to which they: 1) believe that their accommodation is reasonable; 2) think that their accommodation is helpful for them or the individual with the hoarding problem; and 3) think that they need to increase or decrease their 
level of accommodation, on a 5-point scale, ranging from 0 (not at all) to 4 (extremely), to assess their beliefs about the utility of their accommodating behaviours.

\section{Hoarding Rating Scale (HRS; Tolin, Frost, et al., 2010; Tolin, Frost, Steketee, Gray,}

et al., 2008). The HRS consists of five items that assess the features of hoarding (i.e., difficulty discarding, excessive acquisition, clutter, emotional distress, and functional impairment). Each item is rated on a 9-point scale, ranging from 0 (not at all) to 8 (extreme). The five items are summed to yield a total score. The HRS can be administered as a semistructured clinician-rated interview (HRS-I; Tolin, Frost, et al., 2010) or as a self-report questionnaire (HRS-SR; Tolin, Frost, Steketee, Gray, et al., 2008). Both the HRS-I and HRS-SR have been shown to have strong psychometric properties. The HRS-I has been found to have high internal consistency and testretest reliability, and has been found to be strongly associated with other self-report measures of hoarding (Tolin, Frost, et al., 2010). The HRS-SR has been found to have high internal consistency (Tolin, Frost, Steketee, \& Fitch, 2008), and has been found to have strong correlations with the HRS-I (Tolin, Frost, Steketee, Gray, et al., 2008). In addition, previous research has found $73 \%$ agreement between the HRS-SR and HRS-I when hoarding diagnostic status was compared (Tolin, Frost, Steketee, Gray, et al., 2008). The recommended cutoff score for clinically significant hoarding is 14 (Frost \& Hristova; 2011), and the recommended criteria for meeting diagnostic hoarding criteria have been defined as a score of four or more on items one and two, and a score of four or more on either item four or five (Tolin, Frost, Steketee, Gray, et al., 2008). For the present study, the HRS-I was used to assess clinician-rated hoarding symptom severity, and determine whether hoarding participants' hoarding symptoms met diagnostic criteria for hoarding disorder, as defined by Tolin, Frost, Steketee, Gray, et al., 2008. The DSM-5 diagnostic criteria were not used for the present study because at the time that the 
study was developed and completed, hoarding disorder had yet to be accepted for inclusion in the DSM-5. Internal consistency for the HRS-I was good $(\alpha=.85)$. One research assistant, who was trained on the administration of the HRS-I by the author, coded a subset of interviews $(n=10$ interviews) and an intraclass correlation coefficient was computed between the author's ratings and research assistant's ratings to assess interrater reliability. The intraclass correlation was strong for the total score (.88). The HRS-SR was used to screen for eligibility and assess CSOs' own hoarding symptom severity. Internal consistency for the HRS-SR was excellent $(\alpha=.96)$.

Saving Inventory-Revised (SI-R; Frost, Steketee, \& Grisham, 2004). The SI-R is a 23item self-report questionnaire that includes three subscales that assess the three main features of hoarding: difficulty discarding, excessive acquiring, and clutter. Each item is rated on a 5-point scale, ranging from 0 to 4 . Total SI-R scores range from 0 to 92 . The SI-R has good psychometric properties, including good internal consistency and test-retest reliability (Frost et al., 2004). Strong associations have been found with other self-report and clinician-rated measures of hoarding and the SI-R total and subscales (Frost et al., 2004; Tolin, Frost, et al., 2010). The recommended cutoffs for clinically significant hoarding are as follows: SI-R total = 41, SI-R difficulty discarding $=14$, SI-R excessive acquisition $=9$, and SI-R clutter $=17$ (Frost \& Hristova, 2011). Hoarding participant- and CSO-rated hoarding symptom severity were assessed using the SI-R; the hoarding participants reported on their own hoarding symptom severity, whereas the CSOs reported on their perceptions of the hoarding participant's hoarding symptom severity. Internal consistency for hoarding participant- and CSO-rated SI-R total and subscale scores ranged from good to excellent in the present study ( $\alpha$ s $=.86-.94$ for hoarding participant-rated SI-R total and subscale scores, and $\alpha \mathrm{s}=.84-.94$ for CSO-rated SI-R total and subscale scores). 
Clutter Image Rating (CIR; Frost, Steketee, Tolin, \& Renaud, 2008). The CIR is a pictorial self-report measure that provides a more objective measure of clutter severity. The CIR contains nine photos of three rooms (i.e., kitchen, living room, and bedroom), which progress from a room with no clutter to a room filled with clutter. Participants select the photograph that comes closest to matching the amount of clutter in each room of their home. Scores for each room range from 1 (least cluttered) to 9 (most cluttered). A mean score, ranging from 1 to 9 , of the kitchen, living room, and bedroom is created as a composite score. The CIR has strong psychometric properties, including strong internal consistency, test-retest reliability and interrater reliability (Frost et al., 2008). The recommended cutoff for clinically significant hoarding is a score of 4 on the CIR (Frost \& Hristova, 2011). Hoarding participant- and CSO-rated clutter severity was assessed using the CIR; the hoarding participants reported on their own clutter severity, whereas the CSOs reported on their perceptions of the hoarding participant's clutter severity. Internal consistency for the hoarding participant-rated CIR composite score was found to be good $(\alpha=.80)$, as was the CSO-rated CIR composite score $(\alpha=.88)$.

Obsessive-Compulsive Inventory-Revised (OCI-R; Foa et al., 2004). The OCI-R is an 18-item self-report questionnaire that assesses OCD symptoms across the following six factors: washing, checking, obsessions, mental neutralizing, ordering, and hoarding. Each factor includes three items. Participants rate the extent to which they are bothered or distressed by each item on a 5-point scale from 0 (not at all) to 4 (extremely). The six subscales of the OCI-R have good psychometric properties, including good internal consistency, test-retest reliability and strong associations with other measures of OCD (Abramowitz \& Deacon, 2006; Foa et al., 2004). In addition, the OCI-R has also been found to discriminate OCD from other anxiety disorders (Abramowitz \& Deacon, 2006). Hoarding participants and their CSOs completed the OCI-R; 
both reported on their own OCD symptom severity. Cronbach's alphas for the total and subscale scores of the OCI-R ranged from .72 to .92 in the present study.

Quality of Relationships Inventory (QRI; Pierce, Sarason, \& Sarason, 1991). The QRI is a 25-item self-report questionnaire that assesses support, conflict, and depth across different types of dyads (e.g., family relationships and intimate relationships). The support subscale consists of seven items and assesses perceived availability of social support from a CSO. The conflict subscale consists of 12 items and assesses the extent to which the specific relationship is a source of conflict or ambivalence for an individual. The depth subscale consists of six items and assesses the extent to which an individual perceives the specific relationship as being positive, important, and secure (Pierce et al., 1991). Items are rated on a 4-point scale, ranging from 1 (not at all) to 4 (very much). Subscale scores are calculated by summing the items of each subscale and dividing the score by the total number of items in that specific subscale. The QRI has been shown to have adequate psychometric properties, including acceptable internal consistency and moderate test-retest reliability (Pierce, Sarason, Sarason, Solky-Butzel, \& Nagle, 1997). Hoarding participants and CSOs completed the QRI; both reported on their own relationship satisfaction. In the present study, the internal consistency was good for the support and conflict subscales ( $\alpha=.86$ and $\alpha=.83$, respectively) and acceptable for the depth subscale $(\alpha=.75)$.

Patient Rejection Scale (PRS; Kreisman et al., 1979). The PRS is an 11-item self-report questionnaire that assesses CSOs' rejecting attitudes toward identified patients, and conceptually overlaps with the expressed criticism and hostility components of expressed emotion (Kreisman et al., 1979). Participants rate the extent to which they experience each item on a 3-point scale, ranging from 1 (never) to 3 (often). Five items are reversed scored. All items are summed to 
yield a total score, which can range from 11 to 33 . The PRS has good psychometric properties, including good internal consistency and test-retest reliability (Kreisman et al., 1979), and previous research has documented significant correlations between the PRS and the expressed criticism $(r=.23)$ and hostility $(r=.20)$ components of expressed emotion (Rist \& Watzl, 1989, as cited in Bailer, Rist, Bräuer, \& Rey, 1994). Only CSOs completed the PRS. Internal consistency was acceptable $(\alpha=.74)$ in the present study.

Hoarding Attribution Questionnaire (HAQ). The HAQ is a 6-item self-report questionnaire that was developed specifically for the present study to assess the extent to which CSOs perceive their significant other to be in control of their hoarding symptoms (see Appendix C). CSOs are asked to rate the extent to which they agree or disagree with each item on a 5-point scale, ranging from 1 (strongly disagree) to 5 (strongly agree). Two items are reverse-scored. Total scores are calculated by summing the 6 items. Only CSOs completed the HAQ. Internal consistency of the HAQ was questionable $(\alpha=.68)$ in the present study.

Illness Intrusiveness Ratings Scale (IIRS; Devins, 1994). The IIRS is a 13-item selfreport questionnaire that assesses the extent to which a specific problem causes impairment in various domains of functioning. In the present study, standard instructions (i.e., How much does your illness and/or its treatment interfere with your ...) were altered slightly, such that the phrase "illness and/or its treatment" were replaced with the word "hoarding." Participants rate each item using a 7-point scale, ranging from 1 (not very much) to 7 (very much). A total score is calculated by summing all items, with higher scores indicating greater functional impairment. In addition, items on the IIRS can be categorized into three domains of functioning, including lifestyle (items 1, 2, 4, and 5), activity involvement (items 3 and 10,11,13), and intimate relationships (items 7-9; Bieling, Rowa, Antony, Summerfeldt, \& Swinson, 2001). Given that 
items 6 and 12 have been found to have factor loadings greater than .30 on at least two factors (Bieling et al., 2001), they are not included in the subscale scores; however, they are retained in the total scale. Previous research suggests that the IIRS has strong psychometric properties, including good internal consistency (Bieling et al., 2001; Devins et al., 2001). The IIRS was used to assess hoarding participant- and CSO-rated functional impairment; the hoarding participants reported on their own functional impairment, whereas the CSOs reported on their perceptions of the hoarding participant's functional impairment. In the present study, Cronbach's alphas for hoarding participant-rated total and subscale IIRS scores ranged from .85 to .91 , and Cronbach's alphas for CSO-rated total and subscale IIRS scores ranged from .79 to .90.

\section{Activities of Daily Living in Hoarding (ADL-H; Frost, Hristova, Steketee, \& Tolin,}

in press). The ADL-H is a 15 -item self-report questionnaire that assesses the extent to which hoarding behaviours impact an individual's ability to complete activities of daily living (e.g., preparing food, using the bath, or shower). Items are rated on a 5-point scale, ranging from 1 (can do it easily) to 5 (unable to do). There is also a not applicable (NA) response option, which is to be chosen when items do not apply for an individual. Total scores are computed by averaging all items that are not rated as NA. The ADL-H has been found to have good psychometric properties, including excellent internal consistency and strong test-retest reliability. In addition, the ADL-H has been found to discriminate individuals with hoarding problems from individuals with OCD and community controls (Frost et al., in press). The ADL-H was used to assess hoarding participant- and CSO-rated impairment in activities of daily living; the hoarding participants reported on their own impairment in activities of daily living, whereas the CSOs reported on their perceptions of the hoarding participant's impairment in activities of daily living. 
Internal consistency for the ADL-H was found to be excellent for individuals with hoarding problems $(\alpha=.91)$ and acceptable for CSOs $(\alpha=.70)$ in the present study.

\section{Depression Anxiety Stress Scales-21 item version (DASS-21; Lovibond \& Lovibond,}

1995). The DASS-21 is a 21 -item self-report questionnaire that assesses features of depression, anxiety, and stress. The depression subscale assesses symptoms associated with dysphoric mood, the anxiety subscale assesses symptoms associated with physical arousal, and the stress subscale assesses symptoms such as nervous tension, difficulty relaxing, and irritability. The DASS-21 is a short form version of the 42-item Depression Anxiety Stress Scales (Lovibond \& Lovibond, 1995). Participants rate the extent to which they have experienced each symptom over the past week on a 4-point scale, ranging from 0 (not at all) to 3 (most of the time). Scores for the three subscales are calculated by summing the scores for each of the subscale's seven items, and then doubling them. The DASS-21 has strong psychometric properties, including high internal consistency for each subscale and concurrent validity (Antony, Bieling, Cox, Enns, \& Swinson, 1998; Henry \& Crawford, 2005). Hoarding participants and CSOs completed the DASS-21; both reported on their own depression, anxiety, and stress symptom severity. In the present study, internal consistency was excellent for the stress and depression subscales $(\alpha=.91$ and $\alpha=.92$, respectively), and good for the anxiety subscale $(\alpha=.83)$.

Aggression Questionnaire (AQ; Buss \& Warren, 2000). The AQ is a 34-item self-report questionnaire that assesses the following five aspects of trait aggressiveness: anger (i.e., agitation and sense of control), hostility (i.e., resentment, social isolation, and paranoia), verbal aggression (i.e., argumentative and hostile language), physical aggression (i.e., physical expression of anger, and indirect aggression (i.e., expression of anger without direct confrontation). Participants rate the extent to which each item is characteristic of them on a 5-point scale, ranging from 1 (not at 
all like me) to 5 (completely like me), with higher scores representing higher levels of aggression. The 15-item short form of the AQ was used for the present study. Each of the five subscales consists of three items, which are summed and then converted to standardized T-scores. A total score is computed by summing all 15 items and then converting the score to a standardized $\mathrm{T}$ score. The 15-item short form has good psychometric properties, including acceptable internal consistency (Buss \& Warren, 2000). The anger and hostility subscales were used in the present study. Hoarding participants and CSOs completed the 15-item short form; both reported on their own levels of anger and hostility. Internal consistency was questionable for the anger subscale ( $\alpha$ $=.64)$ and acceptable for the hostility subscale $(\alpha=.77)$ in the present study.

Anxiety Sensitivity Index (ASI; Peterson \& Reiss, 1993). The ASI is a 16-item selfreport questionnaire that assesses anxiety sensitivity (i.e., fear of anxiety-related symptoms). Participants rate the extent to which they experience each item on a 5-point scale, ranging from 0 (very little) to 4 (very much). All items are summed to yield a total score. The ASI has good psychometric properties, including good internal consistency and satisfactory test-retest reliability (Peterson \& Reiss, 1993). The ASI was used to assess the discriminant validity of the FAI-H. Hoarding participants and CSOs completed the ASI; both reported on their own level of anxiety sensitivity. In the present study, internal consistency of the ASI was excellent ( $\alpha=.91)$.

\section{Procedure}

Participants were recruited through the community using flyers and newspaper advertisements (see Figure 1, which outlines the flow of participants through the present study). Participants self-identified for the study by contacting the author via telephone or email. When a participant contacted the author, he or she was given more information about the study and both members of the dyad were asked to complete the HRS-SR online to determine eligibility. If 
541 Individuals inquired about the study via email or telephone

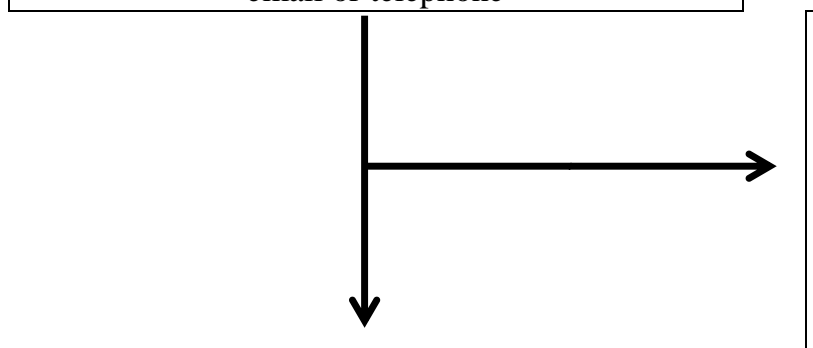

190 Dyads completed the pre-screen questionnaire

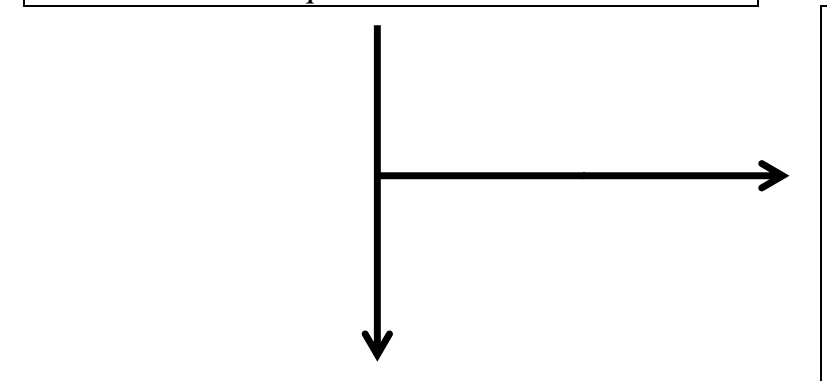

97 dyads were eligible to participate in the study

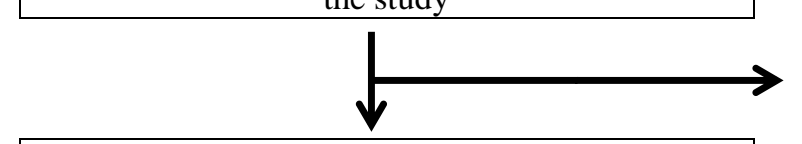

65 Dyads consented to participate in the study

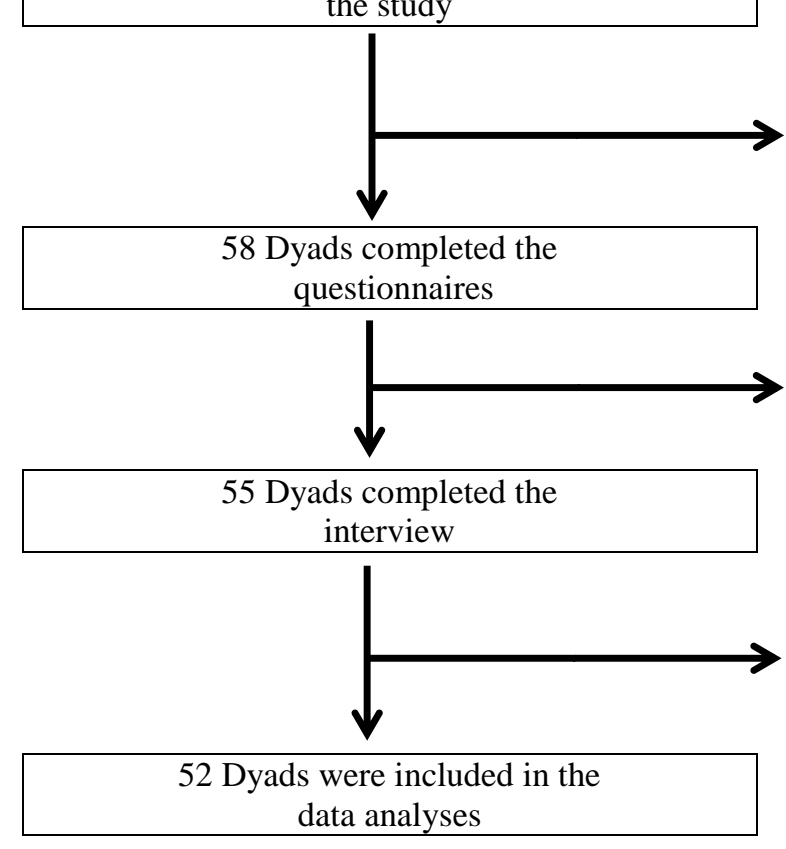

351 Dyads excluded for the following reasons:

- Did not respond or were not interested $(n=244)$

- One member was unwilling to participate $(n=40 ; 20$ were hoarding participants, 19 were CSOs, 1 unknown)

- Hoarding participant did not have a CSO $(n=24)$

- Hoarding participant was no longer living $(n=1)$

- One member was not fluent in English $(n=2)$

- One member did not complete the pre-screen questionnaire $(n=40)$

93 Dyads excluded for the following reasons:

- Both members scored $\geq 14$ on the HRS-I $(n=65)$

- Both members scored $<14$ on the HRS-I $(n=17)$

- The members did not live together or spend at least 4 hours per week together $(n=7)$

- One member was not between 18-75 years of age $(n=2)$

- Did not have regular access to a computer (could not complete the questionnaires; $n=1$ )

- One member was deaf (could not complete the interview; $n=1$ )

32 Dyads excluded for the following reasons:

- Did not complete an informed consent meeting $(n=31)$

- Elected not to provide consent $(n=1)$

7 Dyads excluded for the following reasons:

- One member did not complete the questionnaires $(n=5$; 2 were hoarding participants, 3 were CSOs)

- Both members did not complete the questionnaires $(n=$ 2)

3 Dyads excluded for the following reasons:

- One member did not complete the interview $(n=2 ; 1$

hoarding participant, 1 CSO)

- Both members did not complete the interview $(n=1)$

3 Dyads excluded for the following reasons:

- One member endorsed psychotic symptoms $(n=1)$

- Members were given the incorrect questionnaires $(n=1)$

- Both members met diagnostic criteria for hoarding, as assessed with the HRS-I $(n=1)$

Figure 1. Flow of participants through the present study. CSO = Close significant other. HRS-I = Hoarding Rating ScaleInterview (Tolin, Frost, et al., 2010). 
eligible, the author reviewed the consent agreement form with the dyad via telephone, and each member of the dyad signed the consent form electronically via the Internet. Once the consent form was electronically signed, each member of the dyad was invited to complete the self-report questionnaires online via Qualtrics survey software (Qualtrics, 2010) and the clinician-rated interviews via telephone. Several studies have indicated that Internet data collection yields comparable results to those using the paper-and-pencil format (Coles, Cook, \& Blake, 2007; Gosling, Vazire, Srivastava, \& John, 2004). The clinician-rated interviews were completed by the author and supervised by a registered psychologist. Each participant's assessment, including the clinician-rated interviews and self-report questionnaires, took approximately 3 hours to complete. Each participant received a debriefing form via email following the completion of the clinician-rated interviews. Each member of the dyad received $\$ 40$ for completing the study.

\section{Data Analysis}

Prior to completing analyses, data were screened for violations of statistical assumptions (Field, 2005). In addition, missing values were estimated using mean substitution (Tabachnick \& Fidell, 2007). That is, if less than $20 \%$ of a person's data was missing, the missing values were replaced with the person's mean score for that measure. If more than $20 \%$ of a person's data was missing for a measure, then that person was not included in analyses that included that specific measure. A significance level of $p<.05$ was used for all statistical tests.

The first aim of the study was to assess the internal consistency, interrater reliability, convergent validity, and discriminant validity of the FAI-H adapted specifically for this study. Internal consistency was evaluated using Cronbach's alpha. One research assistant, who was trained on the administration of the FAI-H by the author, coded a subset of interviews $(n=10$ interviews) and intraclass correlation coefficients (ICCs) were calculated between the author's 
ratings and the research assistant's ratings for each item, and the total and subscale scores to assess interrater reliability. Pearson correlations were calculated between the FAI-H total and subscale scores and the CSO-rated FRSH total and subscale scores to investigate the convergent validity of the FAI-H, and between the FAI-H total and subscale scores and the hoarding participant-rated DASS-21 stress subscale scores and hoarding participant-rated ASI scores to investigate the discriminate validity of the FAI-H. Williams' $T_{2}$ tests were conducted to assess the extent to which the convergent and discriminant associations were significantly different from one another.

The second aim of the study was to explore the nature of family accommodation in hoarding by exploring the frequency and severity of family accommodation and assessing CSOs' motivations for, and attitudes about, engaging in accommodating behaviours. The frequency of family accommodation in hoarding was evaluated by assessing the number of CSOs who endorsed at least some accommodation of hoarding symptoms. The severity of family accommodation was explored by examining the range and distribution of FAI-H scores. Independent samples $t$-tests were conducted to assess the extent to which FAI-H total and subscale scores differed for intimate partners of hoarding participants versus other types of dyads, as well as CSOs who lived with the hoarding participant versus CSOs who did not live with the hoarding participant. Pearson correlations were computed between family accommodation and CSOs' own hoarding and OCD symptoms to assess the extent to which these symptoms were related to the CSOs' level of accommodation.

The frequency with which CSOs endorsed various hoarding participant-driven and personally-driven motivations to at least a moderate degree were examined. Pearson correlations were computed between scores on the FAI-H and scores on the hoarding participant-driven 
motivation subscale and the personally-driven motivation subscale to assess the strength of the association between family accommodation and hoarding participant-driven, as well as personally-driven, motivations for engaging in accommodating behaviours. Lastly, the frequency with which CSOs endorsed various attitudes about engaging in accommodating behaviours to at least a moderate degree was also examined.

The third aim of the study was to investigate the construct of family accommodation in hoarding by examining the association between CSOs' accommodating behaviours and hoarding participant- and CSO-rated hoarding symptom severity, relationship satisfaction, hoarding participant- and CSO-rated functional impairment, CSOs' attribution of their significant others' control over hoarding behaviours, CSOs' rejecting attitudes toward the hoarding participant, and hoarding participants' and CSOs' wellbeing. Hoarding symptom severity was assessed with hoarding participant- and CSO-rated SI-R and CIR. Relationship satisfaction was assessed with the QRI. Functional impairment was assessed with the hoarding participant- and CSO-rated IIRS and ADL-H. CSOs' attributions about the hoarding participant's control over hoarding behaviours were assessed with the HAQ. CSOs' level of rejecting attitudes toward the hoarding participant was assessed with the PRS. Hoarding participants' and CSOs' wellbeing was assessed with the depression, anxiety, and stress subscales of the DASS-21, and anger and hostility subscales of the AQ. Pearson correlations were computed to assess the extent to which the FAI-H total and subscale scores were associated with these various measures.

The fourth aim of the study was to investigate the extent to which family accommodation mediated the association between hoarding symptom severity and relationship satisfaction, as well as the association between hoarding symptom severity and functional impairment. Family accommodation was assessed with the CSO and hoarding participant-rated FAI-H total score. 
Hoarding symptom severity was assessed with the CSO- and hoarding participant-rated SI-R total score. Relationship satisfaction was assessed with the CSO- and hoarding participant-rated QIR support, conflict, and depth subscales. Functional impairment was assessed with the CSOand hoarding participant-rated ADL-H. Mediation was assessed using the actor-partner interdependence mediation model (APIMeM; Ledermann, Macho, \& Kenny, 2011), which is an extension of the actor-partner interdependence model (APIM; Kenny, Kashy, \& Cook, 2006).

Actor-Partner Interdependence Model (APIM). A full description of the APIM is provided in Kenny et al. (2006); a brief summary is provided below. The APIM assesses the extent to which an individual's score on a predictor variable is associated with his or her own score on an outcome variable (actor effect) and his or her partner's score on an outcome variable (partner effect); in this case, "partner" refers to the second member of a dyad. For example, in the present study, the actor effect refers to the association between one's own report of the hoarding participant's hoarding symptom severity and one's own report of his or her relationship satisfaction, and the partner effect refers to the association between one's own report of the hoarding participant's hoarding symptom severity and his or her CSO's report of his or her relationship satisfaction. The APIM is displayed in Figure 2. If members of the dyad are distinguishable (i.e., there is a meaningful factor that can be used to differentiate the two members of the dyad), then there are potentially two actor effects, one for dyad member 1 (symbolized as actor $_{\mathrm{H}}$ in Figure 2) and one for dyad member 2 (symbolized as actor $\mathrm{CSO}$ in Figure 2), and two partner effects, one from dyad member 1 to dyad member 2 (symbolized as partner $_{\mathrm{CSO}}$ in Figure 2) and one from dyad member 2 to dyad member 1 (symbolized as partner $_{\mathrm{H}}$ in Figure 2). In the present study, members of the dyad can be distinguished by participant status (i.e., hoarding participant versus CSO). The APIM with distinguishable dyads can be estimated 


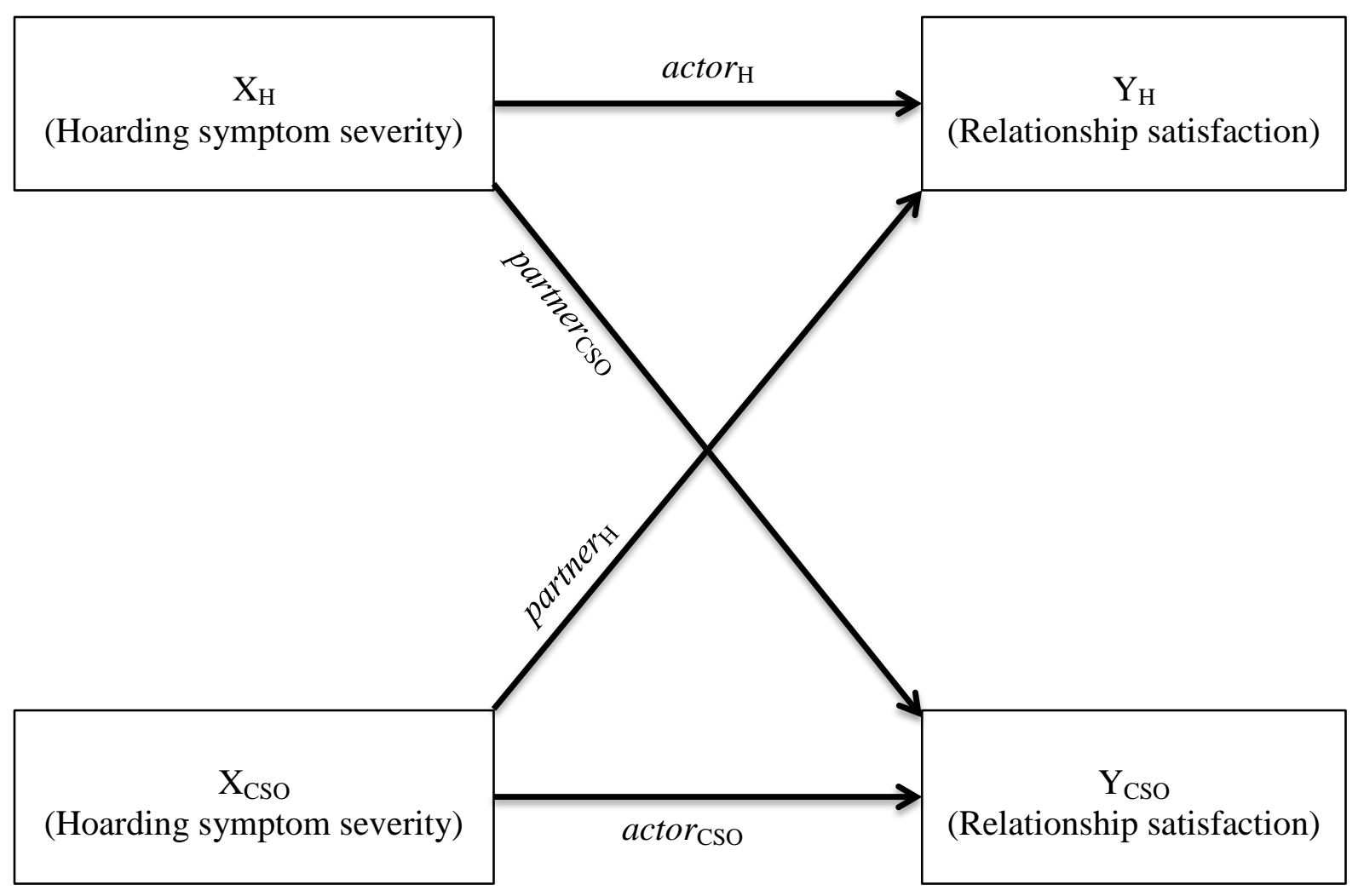

Figure 2. Actor-partner interdependence model (APIM). X = predictor variable (e.g., hoarding symptom severity). $\mathrm{Y}=$ outcome variable (e.g., relationship satisfaction). actor $=$ Actor effect. partner $=$ Partner effect. $\mathrm{H}=$ Dyad member 1 (i.e., hoarding participant). $\mathrm{CsO}=$ Dyad member 2 (i.e., close significant other). Adapted from Kenny, Kashy, \& Cook, 2006. 
using two different multilevel modeling (MLM) approaches (i.e., the interaction model and the two-intercept model; Kenny et al., 2006).

Interaction model. A full description of the interaction model is provided in Kenny et al. (2006); a brief summary is provided below. In the interaction model, the actor and partner effects for the predictor variables, the distinguishing variable, and the interactions between the distinguishing variable and the actor and partner scores on the predictor variable are entered as fixed effects. This model is typically computed first to assess the extent to which the actor and partner effects significantly differ according to the distinguishing variable. In other words, this model assesses the extent to which the actor and partner effects are significant, averaging across dyad members, and the extent to which these effects differ according to the distinguishing variable (Kenny et al., 2006).

Two-intercept model. A full description of the two-intercept model is provided in Kenny et al. (2006); a brief summary is provided below. In the two-intercept model, the distinguishing variable is coded as a repeated measures variable. This model is computed to assess the extent to which the actor and partner effects are significantly different from zero for each member of the dyad. The two-intercept model is typically computed second, after the intercept model, and is only necessary if the actor and partner effects significantly differ according to the distinguishing variable in the intercept model (Kenny et al., 2006).

Actor-Partner Interdependence Mediation Model (APIMeM). A full description of the APIMeM is provided in Ledermann et al. (2011); a brief summary is provided below. The APIMeM extends the APIM by adding a third mediating variable to the model (see Figure 3 for the basic APIMeM). The model consists of two dyad members, two predictor variables (X), two mediating variables $(\mathrm{M})$, and two outcome variables (Y). Overall, the model consists of six actor 


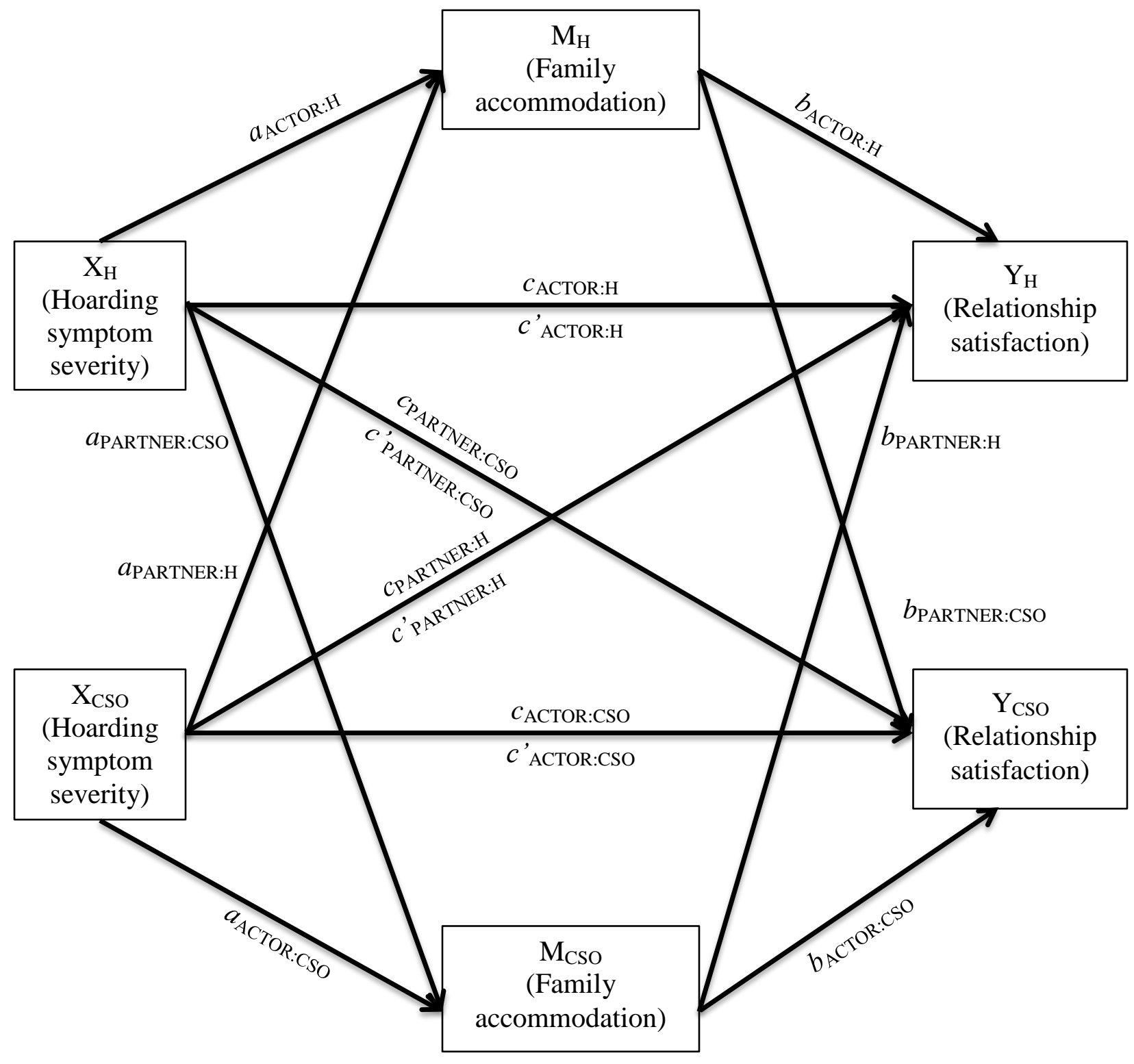

Figure 3. Actor-partner interdependence mediation model (APIMeM). X = Predictor variable (e.g., hoarding symptom severity). $\mathrm{M}=$ Mediator variable (e.g., family accommodation). $\mathrm{Y}=$ Outcome variable (e.g., relationship satisfaction). ${ }_{A C T O R}=$ Actor effect. PARTNER $=$ Partner effect. $a=$ Path a. $b=$ Path b. $c=$ Path c. $c^{\prime}=$ Path c'. $\mathrm{H}_{\mathrm{H}}=$ Dyad member 1 (i.e., hoarding participant). CSO $=$ Dyad member 2 (i.e., close significant other). Adapted from Ledermann, Macho, \& Kenny, 2011. 
effects (symbolized as ${ }_{A C T O R}$ in Figure 3) and six partner effects (symbolized as PARTNER in Figure 3). There are four effects in the APIMeM that could possibly be mediated: the first member's actor effect $\left(\mathrm{X}_{\mathrm{H}} \rightarrow \mathrm{Y}_{\mathrm{H}}\right)$, the second member's actor effect $\left(\mathrm{X}_{\mathrm{CSO}} \rightarrow \mathrm{Y}_{\mathrm{CSO}}\right)$, the first member's partner effect $\left(\mathrm{X}_{\mathrm{CSO}} \rightarrow \mathrm{Y}_{\mathrm{H}}\right)$, and the second member's partner effect $\left(\mathrm{X}_{\mathrm{H}} \rightarrow \mathrm{Y}_{\mathrm{CSO}}\right)$.

However, given that each of these actor and partner effects could be mediated by either member's mediating variable, there are actually eight possible paths of mediation that can be tested for distinguishable dyads. That is, the first member's actor effect could be mediated by his or her own mediating variable $\left(\mathrm{X}_{\mathrm{H}} \rightarrow \mathrm{M}_{\mathrm{H}} \rightarrow \mathrm{Y}_{\mathrm{H}}\right)$, or the second member's mediating variable $\left(\mathrm{X}_{\mathrm{H}} \rightarrow \mathrm{M}_{\mathrm{CSO}} \rightarrow \mathrm{Y}_{\mathrm{H}}\right)$. The second member's actor effect could be mediated by his or her own mediating variable $\left(\mathrm{X}_{\mathrm{CSO}} \rightarrow \mathrm{M}_{\mathrm{CSO}} \rightarrow \mathrm{Y}_{\mathrm{CSO}}\right)$, or the first member's mediating variable $\left(\mathrm{X}_{\mathrm{CSO}} \rightarrow\right.$ $\left.\mathrm{M}_{\mathrm{H}} \rightarrow \mathrm{Y}_{\mathrm{CSO}}\right)$. The first member's partner effect could be mediated by his or her own mediating variable $\left(\mathrm{X}_{\mathrm{CSO}} \rightarrow \mathrm{M}_{\mathrm{H}} \rightarrow \mathrm{Y}_{\mathrm{H}}\right)$, or the second member's mediating variable $\left(\mathrm{X}_{\mathrm{CSO}} \rightarrow \mathrm{M}_{\mathrm{CSO}} \rightarrow \mathrm{Y}_{\mathrm{H}}\right)$. The second member's partner effect could be mediated by his or her own mediating variable $\left(\mathrm{X}_{\mathrm{H}}\right.$ $\left.\rightarrow \mathrm{M}_{\mathrm{CSO}} \rightarrow \mathrm{Y}_{\mathrm{CSO}}\right)$, or the first member's mediating variable $\left(\mathrm{X}_{\mathrm{H}} \rightarrow \mathrm{M}_{\mathrm{H}} \rightarrow \mathrm{Y}_{\mathrm{CSO}}\right.$ Ledermann et al., 2011).

Tests of mediation in the APIMeM consist of four steps, which follow from Baron and Kenny's (1986) steps of mediation. Each step is assessed using MLM. The first step of mediation involves testing the association between the predictor variable and the mediating variable (path $a$; symbolized as $a$ in Figure 3). The second step of mediation involves testing the association between the mediating variable and the outcome variable (path $b$; symbolized as $b$ in Figure 3 ). The third step of mediation involves testing the association between the predictor variable and the outcome variable (path $c$; symbolized as $c$ in Figure 3). The fourth step of mediation involves testing the association between the predictor variable and the outcome variable after accounting 
for the mediating variable (path c'; symbolized as c' in Figure 3). The Sobel test is used to assess whether the mediating variable has mediated the association between the predictor variable and the outcome variable. Mediation is said to have occurred when the indirect effects (i.e., path $a b$ ) are significant and the $c$ ' path is significantly less than the $c$ path. In a mediation model, effects can be completely, partially, or inconsistently mediated. Complete mediation occurs when the $a b$ path is significant and the $c^{\prime}$ path is nonsignificant. Partial mediation occurs when the $a b$ path and the c' path are both significant and have the same sign. Inconsistent mediation occurs when the $a b$ path and the $c^{\prime}$ path are both significant and have opposite signs (Ledermann et al., 2011). 


\section{Results}

Demographic characteristics are provided in Table 1 . The majority of hoarding participants and their CSOs were female and Caucasian. The majority of dyads lived together and identified themselves as intimate partners. Fifty percent of the sample was employed at least part-time, and the majority of individuals reported making $\$ 24,999$ or less per year. Thirty-six hoarding participants (69.23\%) reported clinically significant symptoms of hoarding that met diagnostic criteria for hoarding disorder, as defined by Tolin, Frost, Steketee, Gray, et al. (2008). The most common comorbid diagnosis was major depressive disorder, followed by social anxiety disorder, generalized anxiety disorder, panic disorder, and obsessive-compulsive disorder.

\section{Descriptive Statistics}

Means, standard deviations, and range of scores for each measure by participant status are provided in Table 2. In addition, standardized scores for skewness and kurtosis and results of significance testing for skewness and kurtosis are also displayed in Table 2. The skewness and kurtosis values for the total and subscale scores of the FAI-H are discussed as part of study aim \#2. Although some of the measures violated the assumption of normality, Pearson $r$ is robust against such violations of normality and can be used in situations in which samples of scores are nonnormal (Havlicek \& Peterson, 1977); thus, the violations of normality that were found in the present study are unlikely to influence the distribution of $r$ s and results of the Pearson correlations are likely valid.

\section{Study Aim \#1}

The first aim of the study was to assess the internal consistency, interrater reliability, convergent validity, and discriminant validity of the FAI-H. Given that the FAS for OCD, which is the measure that the FAI-H was adapted from, has demonstrated strong psychometric 
Table 1

Demographic Characteristics by Participant Status

\begin{tabular}{|c|c|c|c|}
\hline & $\begin{array}{l}\text { Overall sample } \\
\quad(N=104)\end{array}$ & $\begin{array}{c}\text { Hoarding participant } \\
\quad(n=52)\end{array}$ & $\begin{array}{l}\text { Close significant other } \\
\qquad(n=52)\end{array}$ \\
\hline Characteristic & $M$ or $\mathrm{n}(S D$ or $\%)$ & $M$ or $\mathrm{n}(S D$ or $\%)$ & $M$ or $\mathrm{n}(S D$ or $\%)$ \\
\hline Male & $33(32 \%)$ & $8(15 \%)$ & $25(48 \%)$ \\
\hline Caucasian & $67(66 \%)$ & $34(67 \%)$ & $33(65 \%)$ \\
\hline Intimate partner & $58(56 \%)$ & $29(56 \%)$ & $29(56 \%)$ \\
\hline Parent & $17(16 \%)$ & $13(25 \%)$ & $4(8 \%)$ \\
\hline Child & $17(16 \%)$ & $4(8 \%)$ & $13(25 \%)$ \\
\hline Sibling & $8(8 \%)$ & $4(8 \%)$ & $4(8 \%)$ \\
\hline \multicolumn{4}{|l|}{ Live together } \\
\hline Yes & $74(71 \%)$ & $37(71 \%)$ & $37(71 \%)$ \\
\hline No & $30(29 \%)$ & $15(29 \%)$ & $15(29 \%)$ \\
\hline $\begin{array}{l}\text { If no, average number of } \\
\text { hours spent together/week }\end{array}$ & $15(13)$ & $15(14)$ & $15(14)$ \\
\hline Employed at least part-time & $52(50 \%)$ & $23(44 \%)$ & $29(56 \%)$ \\
\hline
\end{tabular}


Individual annual income

Under $\$ 5,000$

$17(18 \%)$

$9(19 \%)$

$8(17 \%)$

$\$ 5,000$ to $\$ 9,999$

$8(9 \%)$

$3(6 \%)$

$5(11 \%)$

$\$ 10,000$ to $\$ 14,999$

$16(17 \%)$

$10(21 \%)$

$6(13 \%)$

$\$ 15,000$ to $\$ 24,999$

$7(7 \%)$

$6(13 \%)$

$1(2 \%)$

$\$ 25,000$ to $\$ 34,999$

$8(9 \%)$

$5(11 \%)$

$3(6 \%)$

$\$ 35,000$ to $\$ 49,999$

$11(12 \%)$

$2(4 \%)$

$9(19 \%)$

$13(14 \%)$

$6(13 \%)$

$7(15 \%)$

$\$ 75,000$ to $\$ 99,999$

$5(11 \%)$

$5(11 \%)$

$\$ 100,000$ to $\$ 249,999$

$1(2 \%)$

$3(6 \%)$

Current co-morbid diagnoses

Major depressive disorder

Panic disorder

$11(11 \%)$

$5(5 \%)$

$8(15 \%)$

$3(6 \%)$

Social phobia

$10(10 \%)$

$4(8 \%)$

$1(2 \%)$

Obsessive-compulsive disorder

$6(6 \%)$

$8(15 \%)$

$2(4 \%)$

Posttraumatic stress disorder

Generalized anxiety disorder

$7(7 \%)$

$2(2 \%)$

$0(0 \%)$

$1(2 \%)$

Alcohol dependence

$7(13 \%)$

$0(0 \%)$

Alcohol abuse

$1(1 \%)$

$1(2 \%)$

$1(2 \%)$

$2(2 \%)$

$0(0 \%)$

$1(2 \%)$

Substance abuse

$0(0 \%)$

$2(4 \%)$

Lifetime co-morbid diagnoses Major depressive disorder 
Table 2

Descriptive Statistics by Participant Status

\begin{tabular}{|c|c|c|c|c|c|c|c|c|c|c|}
\hline \multirow[b]{2}{*}{ Measure } & \multicolumn{5}{|c|}{ Hoarding participant } & \multicolumn{5}{|c|}{ Close significant other } \\
\hline & $n$ & $M(S D)$ & Range & Skewness & Kurtosis & $n$ & $M(S D)$ & Range & Skewness & Kurtosis \\
\hline \multicolumn{11}{|l|}{ Family accommodation } \\
\hline FAI-H total & 52 & $\begin{array}{c}20.45 \\
(16.67)^{b}\end{array}$ & $\begin{array}{l}0.00- \\
63.00\end{array}$ & 1.93 & -0.75 & 52 & $\begin{array}{c}22.94 \\
(16.46)\end{array}$ & $\begin{array}{l}0.00- \\
67.00\end{array}$ & $2.59^{* *}$ & 0.24 \\
\hline FAI-H frequency & 52 & $\begin{array}{c}12.06 \\
(8.95)^{b}\end{array}$ & $\begin{array}{l}0.00- \\
29.00\end{array}$ & 1.07 & -1.63 & 52 & $\begin{array}{l}11.83 \\
(7.58)\end{array}$ & $\begin{array}{l}0.00- \\
31.00\end{array}$ & $2.24^{*}$ & 0.39 \\
\hline FAI-H distress/interference & 52 & $\begin{array}{c}8.39 \\
(8.61)^{b}\end{array}$ & $\begin{array}{l}0.00- \\
34.00\end{array}$ & $3.21^{* *}$ & 0.57 & 52 & $\begin{array}{l}11.12 \\
(9.38)\end{array}$ & $\begin{array}{l}0.00- \\
36.00\end{array}$ & $2.72^{* *}$ & 0.03 \\
\hline FRHS total & & & & & & 51 & $\begin{array}{c}14.81 \\
(11.65)\end{array}$ & $\begin{array}{l}0.00- \\
48.00\end{array}$ & $2.23^{*}$ & -0.22 \\
\hline $\begin{array}{l}\text { FRHS family } \\
\text { behaviour changes }\end{array}$ & & & & & & 51 & $\begin{array}{c}8.22 \\
(6.82)\end{array}$ & $\begin{array}{l}0.00- \\
29.00\end{array}$ & $2.80^{* *}$ & 0.71 \\
\hline FRHS family consequences & & & & & & 52 & $\begin{array}{c}4.21 \\
(3.78)\end{array}$ & $\begin{array}{l}0.00- \\
12.00\end{array}$ & 1.64 & -1.61 \\
\hline \multicolumn{11}{|l|}{ Hoarding symptom severity } \\
\hline HRS-I & 52 & $\begin{array}{l}20.98 \\
(7.77)\end{array}$ & $\begin{array}{l}5.00- \\
35.00\end{array}$ & -1.15 & -1.42 & & & & & \\
\hline HRS-SR & 52 & $\begin{array}{l}24.00 \\
(8.10)\end{array}$ & $\begin{array}{l}4.00- \\
40.00\end{array}$ & -0.14 & -0.33 & 51 & $\begin{array}{c}3.67 \\
(4.19)\end{array}$ & $\begin{array}{l}0.00- \\
16.00\end{array}$ & $4.03^{* * *}$ & 1.83 \\
\hline SI-R total & 52 & $\begin{array}{c}55.25 \\
(16.12)\end{array}$ & $\begin{array}{l}7.00- \\
88.00\end{array}$ & -0.25 & 0.70 & 52 & $\begin{array}{c}58.68 \\
(15.82)^{\mathrm{a}}\end{array}$ & $\begin{array}{c}27.00- \\
92.00\end{array}$ & 0.02 & -1.15 \\
\hline SI-R clutter & 52 & $\begin{array}{l}20.69 \\
(8.02)\end{array}$ & $\begin{array}{l}3.00- \\
36.00\end{array}$ & -0.49 & -1.31 & 52 & $\begin{array}{c}21.62 \\
(7.74)^{\mathrm{a}}\end{array}$ & $\begin{array}{l}6.00- \\
36.00\end{array}$ & -0.25 & -1.08 \\
\hline
\end{tabular}




\begin{tabular}{|c|c|c|c|c|c|c|c|c|c|c|}
\hline SI-R difficulty discarding & 52 & $\begin{array}{l}19.33 \\
(4.86)\end{array}$ & $\begin{array}{l}4.00- \\
28.00\end{array}$ & -0.97 & 1.15 & 52 & $\begin{array}{c}20.56 \\
(4.60)^{\mathrm{a}}\end{array}$ & $\begin{array}{c}11.00- \\
28.00\end{array}$ & -0.32 & -1.51 \\
\hline SI-R acquisition & 52 & $\begin{array}{l}15.23 \\
(5.79)\end{array}$ & $\begin{array}{l}0.00- \\
27.00\end{array}$ & 0.41 & 0.05 & 52 & $\begin{array}{c}16.50 \\
(5.48)^{\mathrm{a}}\end{array}$ & $\begin{array}{l}3.00- \\
28.00\end{array}$ & -0.56 & -0.55 \\
\hline CIR & 52 & $\begin{array}{c}3.10 \\
(1.43)\end{array}$ & $\begin{array}{c}1.00- \\
7.67\end{array}$ & $2.68^{* *}$ & 1.10 & 52 & $\begin{array}{c}3.51 \\
(1.64)^{\mathrm{a}}\end{array}$ & $\begin{array}{l}1.00- \\
9.00\end{array}$ & $3.01^{* *}$ & $2.20^{*}$ \\
\hline \multicolumn{11}{|l|}{ Relationship satisfaction } \\
\hline QRI support & 52 & $\begin{array}{c}3.21 \\
(0.57)\end{array}$ & $\begin{array}{c}2.00- \\
4.00\end{array}$ & -0.98 & -1.45 & 52 & $\begin{array}{l}3.16 \\
(0.67)\end{array}$ & $\begin{array}{c}1.43- \\
4.00\end{array}$ & -1.95 & -0.66 \\
\hline QRI conflict & 51 & $\begin{array}{c}2.10 \\
(0.54)\end{array}$ & $\begin{array}{c}1.08- \\
3.45\end{array}$ & 0.91 & -0.34 & 52 & $\begin{array}{c}2.28 \\
(0.57)\end{array}$ & $\begin{array}{c}1.25- \\
3.67\end{array}$ & 1.22 & 0.26 \\
\hline QRI depth & 51 & $\begin{array}{c}3.46 \\
(0.43)\end{array}$ & $\begin{array}{c}2.00- \\
4.00\end{array}$ & $-3.02^{* *}$ & $2.00^{*}$ & 52 & $\begin{array}{c}3.37 \\
(0.55)\end{array}$ & $\begin{array}{c}2.00- \\
4.00\end{array}$ & -1.78 & -0.85 \\
\hline PRS & & & & & & 52 & $\begin{array}{l}16.94 \\
(3.64)\end{array}$ & $\begin{array}{c}11.00- \\
27.00\end{array}$ & 0.85 & -0.54 \\
\hline HAQ & & & & & & 51 & $\begin{array}{l}18.87 \\
(4.43)\end{array}$ & $\begin{array}{l}9.60- \\
30.00\end{array}$ & 0.01 & -0.07 \\
\hline \multicolumn{11}{|l|}{ Functional impairment } \\
\hline IIRS & 52 & $\begin{array}{c}42.27 \\
(19.45)\end{array}$ & $\begin{array}{c}13.00- \\
83.91\end{array}$ & 1.14 & -1.13 & 51 & $\begin{array}{c}44.42 \\
(18.12)^{\mathrm{a}}\end{array}$ & $\begin{array}{c}15.00- \\
83.00\end{array}$ & 0.26 & -1.59 \\
\hline IIRS lifestyle & 52 & $\begin{array}{l}11.83 \\
(6.56)\end{array}$ & $\begin{array}{l}4.00- \\
27.00\end{array}$ & $2.03^{*}$ & -0.92 & 52 & $\begin{array}{c}13.54 \\
(6.46)^{\mathrm{a}}\end{array}$ & $\begin{array}{l}4.00- \\
28.00\end{array}$ & 0.85 & -1.02 \\
\hline IIRS activity & 52 & $\begin{array}{l}14.01 \\
(7.35)\end{array}$ & $\begin{array}{l}4.00- \\
28.00\end{array}$ & 1.01 & -1.41 & 51 & $\begin{array}{l}14.20 \\
(6.23)^{\mathrm{a}}\end{array}$ & $\begin{array}{l}4.00- \\
28.00\end{array}$ & -0.30 & -1.52 \\
\hline IIRS relationships & 46 & $\begin{array}{l}10.67 \\
(4.99)\end{array}$ & $\begin{array}{l}3.00- \\
21.00\end{array}$ & 1.13 & -1.40 & 45 & $\begin{array}{c}10.86 \\
(5.30)^{\mathrm{a}}\end{array}$ & $\begin{array}{l}3.00- \\
21.00\end{array}$ & 0.27 & -1.94 \\
\hline
\end{tabular}




\section{ADL-H}

Measures of wellbeing

$$
\text { DASS-21 stress }
$$

DASS-21 depression

DASS-21 anxiety

AQ anger

AQ hostility

OCI-R total

OCI-R washing

OCI-R obsessing

OCI-R hoarding

OCI-R ordering

OCI-R checking

OCI-R neutralising
52

$1.87 \quad 1.00-\quad 1.32$

$-0.33$

51

$\begin{array}{cc}2.20 & 1.13- \\ (0.71)^{\mathrm{a}} & 3.93\end{array}$

1.23

$-0.72$

(0.60)

51

$\begin{array}{cc}19.29 & 0.00- \\ (11.00) & 42.00\end{array}$

$-0.31$

$-1.04$

$16.55 \quad 0.00$ -

$2.09^{*}$

1.04

52

11.03 (10.12)

0.00 $2.86^{* *}$

0.82 (12.73) 42.00

$-1.27$
$2.62^{* *}$

42.00

(3.28) $\quad 12.00$
52

$52 \quad 9.8$

$$
\begin{array}{r}
9.81 \\
(8.22)
\end{array}
$$

0.00 -

1.07

$\begin{array}{ccc}52 & 54.81 & 38.00- \\ & (9.94) & 78.00\end{array}$$$
0.56
$$

$-0.83$

$52 \quad 56.44 \quad 37.00-$ (9.33) 76.00

$-0.05$

$-0.63$

$\begin{array}{lcc}52 & 23.42 & 4.00- \\ & (12.71) & 68.00\end{array}$$$
4.48^{* * * *}
$$

$3.97^{* * *}$

4.48

$7.27^{* * *}$

$\begin{array}{ccc}52 & 1.77 & 0.00- \\ & (3.27) & 12.00\end{array}$

$7.08^{* * *}$

7.27

$3.23^{* *}$

0.94

$\begin{array}{ll}3.24) & 12.00\end{array}$

$\begin{array}{lcc}52 & 8.42 & 1.00-\end{array}$

$-1.36$

$-0.82$

$(2.79) \quad 12.00$

$\begin{array}{lll}52 & 4.92 & 0.00-\end{array}$

$1.98^{*}$

$-0.4$

$4.38^{* * *}$

2.54 (3.02) $\quad 12.00$

$6.26^{* * *}$

$6.91^{* * *}$

$49.16 \quad 37.00-$

$\begin{array}{ccc}52 & 3.19 & 0.00- \\ & (3.02) & 12.00\end{array}$

$\begin{array}{ccc}52 & 1.96 & 0.00- \\ & (2.81) & 12.00\end{array}$

(6.91

$\begin{array}{ccc} & (2.77) & 11.00 \\ 50 & 1.54 & 0.00-\end{array}$

$\begin{array}{lll} & (2.62) & 12.00 \\ 50 & 2.08 & 0.00-\end{array}$

52

52

8.96
$(10.50)$

5.50

$(8.17)$

(8.17)

0.00

(8.29) $\quad 65.00$

$51 \quad 49.16 \quad 37.00-$

(9.07) 71.00

$50 \quad 10.43 \quad 0.00-$

$\begin{array}{ll}10.43 & 0.00- \\ (12.12) & 66.00\end{array}$

$\begin{array}{lcc}50 & 1.34 & 0.00- \\ & (2.62) & 12.00\end{array}$

$3.61^{* * *} \quad 0.84$

$$
6.69^{* * *} \quad 7.70^{* * *}
$$$$
0.92 \quad-1.53
$$

$1.25-0.99$

$7.23^{* * *} \quad 12.25^{* * *}$

$7.47^{* * *} \quad 9.57^{* * *}$

$5.07^{* * *} \quad 4.25^{* * *}$

$5.01^{* * *} \quad 5.47^{* * *}$

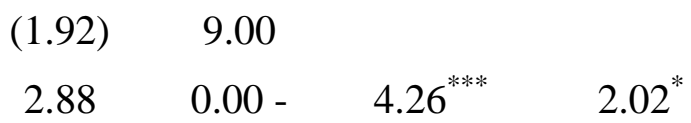

$\begin{array}{ccccc}50 & 2.88 & 0.00- & 4.26^{* * *} & 2.02^{*} \\ & (3.31) & 12.00 & & \end{array}$

$\begin{array}{lllll}50 & 1.58 & 0.00- & 7.97^{* * * *} & 11.39^{* * * *}\end{array}$

$\begin{array}{lllll}50 & 1.00 & 0.00- & 9.04^{* * *} & 17.42^{* * *} \\ & (2.04) & 11.00 & & \end{array}$ 
Notes. FAI-H = Family Accommodation Interview for Hoarding. FRHS = Family Response to Hoarding Scale (Steketee et al., 2013). HRS-I = Hoarding Rating Scale-Interview (Tolin, Frost, et al., 2010). HRS-SR = Hoarding Rating Scale-Self Report (Tolin, Frost, et al., 2010). SI-R = Saving Inventory-Revised (Frost et al., 2004). CIR = Clutter Image Rating (Frost et al., 2008). QRI = Quality of Relationships Inventory (Pierce et al., 1991). PRS = Patient Rejection Scale (Kreisman et al., 1979). HAQ = Hoarding Attribution Questionnaire. IIRS = Illness Intrusiveness Rating Scale (Devins, 1994). ADL-H = Activities of Daily Living in Hoarding Scale (Frost et al., in press). OCI-R = Obsessive-Compulsive Inventory-Revised (Foa et al., 2004). DASS-21 = Depression Anxiety Stress Scales21 item version (Lovibond \& Lovibond, 1995). AQ = Aggression Questionnaire-15 item short form (Buss \& Warren, 2000). ASI = Anxiety Sensitivity Index (Peterson \& Reiss, 1993). ${ }^{\text {a }}=$ CSOs reported on their perceptions of hoarding participants' hoarding symptoms or impairment. ${ }^{\mathrm{b}}=$ Hoarding participants reported on their perceptions of CSOs' behaviours. ${ }^{*} p<.05 .^{* * *} p<.01 .^{* * * *} p<.001$. 
properties, including good internal consistency and excellent interrater reliability (Calvocoressi, Mazure, Stanislav, et al., 1999); it was hypothesized that the FAI-H would also demonstrate strong psychometric properties.

Descriptive statistics (means, standard deviations, corrected item-total correlations) for the FAI-H are provided in Table 3. The corrected item-total correlations for each item were above the recommended cut-off of $r=.30$ (Field, 2005). Internal consistency was evaluated using Cronbach's alpha. Internal consistency for the total score was excellent $(\alpha=.91)$, the frequency subscale was acceptable $(\alpha=.79)$, and the distress/interference subscale was good $(\alpha=.87$; George \& Mallery, 2003). There were two large positively skewed items (i.e., item 5 frequency and item 5 distress/interference); removal of these two items did not improve the scale's internal consistency and thus, the two items were retained as part of the scale. Interrater reliability was evaluated using ICCs for a subset of interviews $(n=10)$. There was almost perfect agreement between the two raters for each item of the FAI-H (see Table 3). The intraclass correlations were strong for the total score (.99), frequency subscale (.99), and distress/interference subscale (.99).

Pearson correlations were conducted between FAI-H total and subscale scores and CSOrated FRHS total and subscale scores to investigate the convergent validity of the FAI-H (see Table 4). The total and subscale scores of the FAI-H were positively and significantly associated with the total and subscale scores of the FRHS. Pearson correlations were conducted between FAI-H total and subscale scores, hoarding participant-rated DASS-21 stress subscale scores, and hoarding participant-rated ASI scores to investigate the discriminate validity of the FAI-H (see Table 5). There was a significant positive association between the frequency subscale scores of the FAI-H and hoarding participant-rated ASI scores; no other associations were significant. 
Table 3

Characteristics of the Family Accommodation Interview for Hoarding (FAI-H; $n=52)$

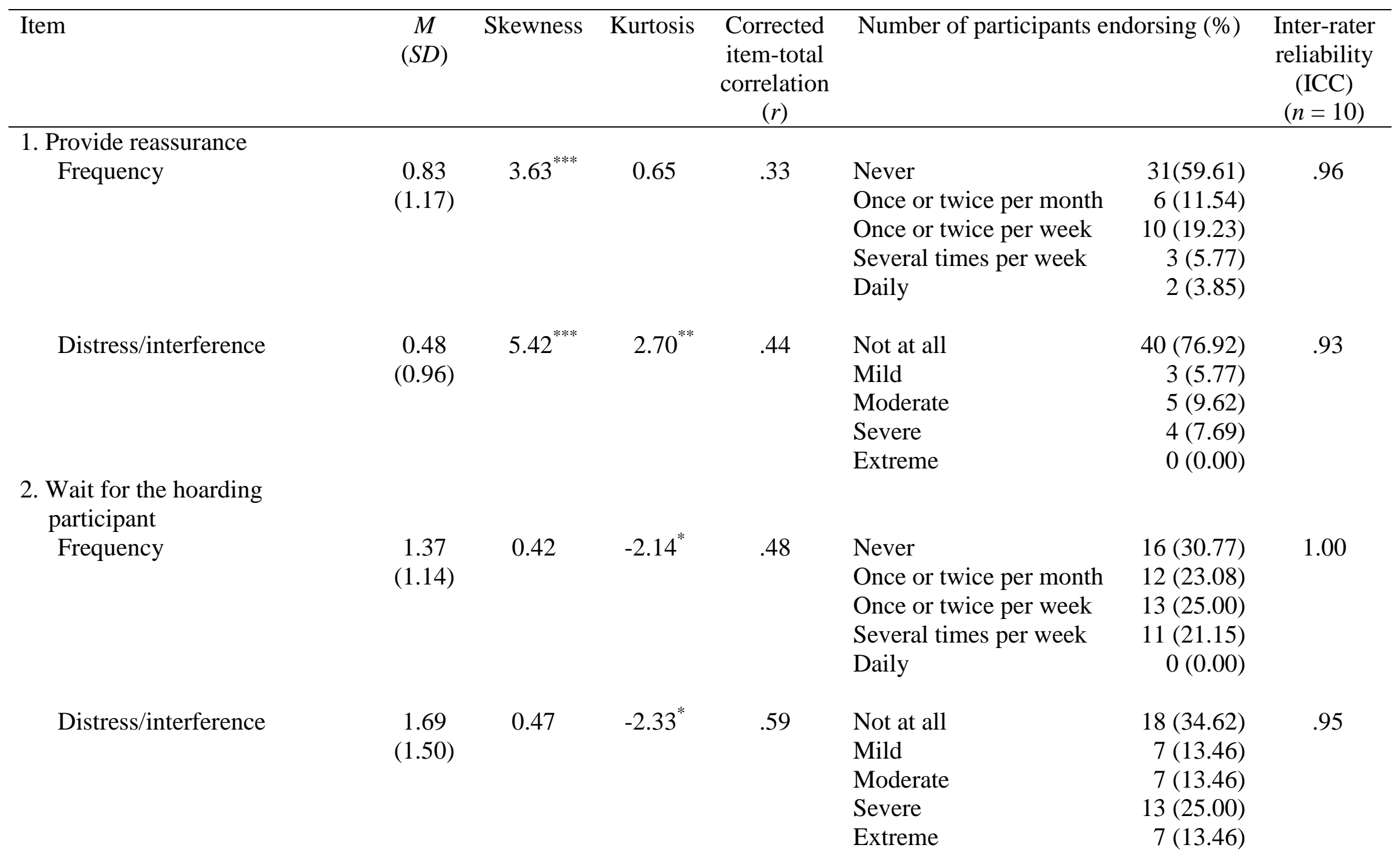


3. Participate in/facilitate the hoarding participant's acquiring

Frequency

$\begin{array}{lll}0.81 & 3.51^{* * *} \quad 1.41\end{array}$

.49

Never

$26(50.00)$

Once or twice per month 14 (26.92)

Once or twice per week 9 (17.31)

Several times per week $2(3.85)$

Daily

$1(1.92)$

Distress/interference

$\begin{array}{ccc}0.94 & 3.29^{* * *} & -0.42 \\ (1.33) & & \end{array}$

.60

Not at all

Mild

Moderate

Severe

$31(59.62)$

$6(11.54)$

$5(9.62)$

$7(13.46)$

Extreme

$3(5.77)$

4. Participate in/facilitate the saving of personal possessions Frequency

$-0.84$

.40

Never

$18(34.62)$

$12(23.08)$

Once or twice per month

Once or twice per week

$13(25.00)$

Several times per week

$2(3.85)$

Daily

7 (13.46)

Distress/interference

1.17

$2.45^{*}$

$-1.37$

.62

Not at all

Mild

Moderate

Severe

Extreme

$27(51.92)$

7 (13.46)

$5(9.62)$

$8(15.38)$

$5(9.62)$

5. Facilitate avoidance

Frequency

$8.89^{* * *} \quad 12.60^{* * *}$

(0.65)

.43

Never

$45(86.54)$

$3(5.77)$

Once or twice per month

$3(5.77)$

Once or twice per week

Several times per week

1 (1.92)

Daily

$0(0.00)$ 


\section{Distress/interference}

6. Tolerate odd behaviours or household disruption

Frequency

Distress/interference

7. Refrain from saying/doing things Frequency

Distress/interference

$\begin{array}{ccc}1.90 & 0.58 & -2.39^{*} \\ (1.62) & & \end{array}$

$0.13 \quad 13.32^{* * *} \quad 31.12^{* * *}$

$$
(0.53)
$$

$(1.62)$

$\begin{array}{lll}1.67 & 0.48 & -2.05^{*}\end{array}$

(1.38)

1.73

(1.60)

$$
0.85
$$

$-2.31^{*}$$$
\text { (1.60) }
$$

$-2.31^{\circ}$

$\begin{array}{lll}1.33 & 1.87 & -1.74\end{array}$

\section{Not at all \\ Mild \\ Moderate \\ Severe}

Extreme

.55

Never

Once or twice per month

Once or twice per week

Several times per week

Daily

.63

Not at all

Mild

Moderate

Severe

Extreme

.52

Never

Once or twice per month Once or twice per week Several times per week Daily

$.69 \quad$ Not at all

Mild

Moderate

Severe

Extreme
48 (92.31)

$2(3.85)$

$1(1.92)$

$1(1.92)$

$0(0.00)$

$15(28.85)$

$9(17.31)$

$10(19.23)$

2 (3.85)

$16(30.77)$

$15(28.85)$

$10(19.23)$

9 (17.31)

13 (25.00)

5 (9.62)

$18(34.62)$

8 (15.38)

8 (15.38)

$6(11.54)$

12 (23.08)

$24(46.15)$

7 (13.46)

7 (13.46)

8 (15.38)

$6(11.54)$ 
8. Make excuses for the hoarding participant's behaviour

Frequency

$\begin{array}{ccc}0.44 & 4.94^{* * *} & 2.56 \\ (0.78) & & \\ & & \\ & & \\ & & \\ 0.63 & 5.00^{* * *} & 1.78 \\ (1.24) & & \end{array}$

Distress/interference

9. Help the hoarding participant with tasks of daily living or simple decisions

Frequency

$$
\begin{gathered}
1.06 \\
(1.30)
\end{gathered}
$$$$
2.50^{*}
$$

$$
2.56^{*}
$$

Never

$37(71.15)$

1.00

$8(15.38)$

$6(11.54)$

Once or twice per week

Several times per week

Daily

$1(1.92)$

$0(0.00)$

Not at all

Mild

$40(76.92)$

$1(1.92)$

Moderate

Severe

$3(5.77)$

$6(11.54)$

Extreme

$2(3.85)$

Never

$28(53.85)$

Once or twice per month Once or twice per week $4(7.69)$

$12(23.08)$

Several times per week

Daily

$5(9.62)$

$3(5.77)$

Distress/interference

$\begin{array}{ccc}0.92 & 3.24^{* *} & -0.34 \\ (1.31) & \end{array}$

.68

Not at all

Mild

$32(61.54)$

$3(5.77)$

Moderate

$9(17.31)$

Severe

$5(9.62)$

Extreme

$3(5.77)$

10. Take on the hoarding participant's responsibilities Frequency 


\begin{tabular}{|c|c|c|c|c|c|c|c|}
\hline \multirow[b]{2}{*}{ Distress/interference } & \multirow[b]{2}{*}{$\begin{array}{c}0.73 \\
(1.37)\end{array}$} & \multirow[b]{2}{*}{$4.83^{* * * *}$} & \multirow[b]{2}{*}{1.51} & \multirow[b]{2}{*}{.69} & \multirow{2}{*}{$\begin{array}{l}\text { Several times per week } \\
\text { Daily } \\
\text { Not at all } \\
\text { Mild } \\
\text { Moderate } \\
\text { Severe } \\
\text { Extreme }\end{array}$} & \multicolumn{2}{|l|}{$\begin{array}{l}2(3.85) \\
4(7.69)\end{array}$} \\
\hline & & & & & & $\begin{array}{r}39(75.00) \\
1(1.92) \\
4(7.69) \\
3(5.77) \\
5(9.62)\end{array}$ & .98 \\
\hline $\begin{array}{l}\text { 11. Modify your personal } \\
\text { family routine } \\
\text { Frequency }\end{array}$ & $\begin{array}{c}1.33 \\
(1.13)\end{array}$ & 0.72 & -1.56 & .67 & $\begin{array}{l}\text { Never } \\
\text { Once or twice per month } \\
\text { Once or twice per week } \\
\text { Several times per week } \\
\text { Daily }\end{array}$ & $\begin{array}{r}17(32.69) \\
10(19.23) \\
17(32.69) \\
7(13.46) \\
1(1.92)\end{array}$ & 1.00 \\
\hline Distress/interference & $\begin{array}{c}1.40 \\
(1.43)\end{array}$ & 1.37 & -1.89 & .70 & $\begin{array}{l}\text { Not at all } \\
\text { Mild } \\
\text { Moderate } \\
\text { Severe } \\
\text { Extreme }\end{array}$ & $\begin{array}{r}22(42.31) \\
6(11.54) \\
10(19.23) \\
9(17.31) \\
5(9.62)\end{array}$ & .93 \\
\hline
\end{tabular}


Table 4

Convergent Validity of the Family Accommodation Interview for Hoarding (FAI-H)

\begin{tabular}{|c|c|c|c|c|c|c|}
\hline Measure & 1 & 2 & 3 & 4 & 5 & 6 \\
\hline 1. FAI-H total & - & & & & & \\
\hline 2. FAI-H frequency & $.96^{* * *}$ & - & & & & \\
\hline 3. FAI-H distress/interference & $.98^{* * *}$ & $.88^{* * *}$ & - & & & \\
\hline 4. FRHS total & $.76^{* * *}$ & $.75^{* * *}$ & $.73^{* * *}$ & - & & \\
\hline 5. FRHS family behaviour changes & $.69^{* * *}$ & $.72^{* * *}$ & $.64^{* * *}$ & $.92^{* * *}$ & - & \\
\hline 6. FRHS family consequences & $.63^{* * *}$ & $.59^{* * *}$ & $.63^{* * *}$ & $.82^{* * *}$ & $.59^{* * *}$ & - \\
\hline
\end{tabular}

Notes. $n \mathrm{~s}=51-52$. FRHS = Family Response to Hoarding Scale (Steketee et al., 2013). ${ }^{\text {**** }} p<.001$. 
Table 5

Discriminant Validity of the Family Accommodation Interview for Hoarding (FAI-H)

\begin{tabular}{llll}
\hline Measure & 1 & 2 & 3 \\
\hline Family accommodation & & \\
1. FAI-H total & - & & \\
2. FAI-H frequency & $.96^{* * *}$ & $.88^{* * *}$ & - \\
3. FAI-H distress/interference & $.98^{* * *}$ & & \\
Hoarding participant-rated symptoms & & .20 & .13 \\
4. DASS-21 stress & .17 & $.28^{*}$ & $.25^{\dagger}$ \\
5. ASI & $.27^{\dagger}$ & $.45^{* *}$ & - \\
\hline
\end{tabular}

Notes. $n \mathrm{~s}=51-52$. DASS-21 = Depression Anxiety Stress Scales-21 item version (Lovibond \& Lovibond, 1995). ASI = Anxiety Sensitivity Index (Peterson \& Reiss, 1993). ${ }^{\dagger} p<.10 .{ }^{*} p<.05 .{ }^{* *} p<.01 .{ }^{* * *} p<.001$. 
Williams' $T_{2}$ tests were computed to assess the extent to which the correlations of the FAI-H total and subscale scores with the FRHS total and subscale scores were significantly different than the correlations of the FAI-H total and subscale scores with hoarding participantrated DASS-21 stress subscale scores, and with hoarding participant-rated ASI scores. The correlations of the FAI-H total and subscale scores with the FRHS total and subscale scores were significantly greater than the correlations of the FAI-H total and subscale scores with hoarding participant-rated DASS-21 stress subscale scores, and with hoarding participant-rated ASI scores $(t \mathrm{~s} \geq 2.35 ; p s<.05)$.

\section{Study Aim \#2}

The second aim of the study was to explore the nature of family accommodation in hoarding by exploring the frequency and severity of family accommodation and assessing CSOs' motivations for, and attitudes about, engaging in accommodating behaviours. Consistent with Steketee et al. (2013), CSOs were hypothesized to endorse accommodating behaviours. Like previous OCD-related research, it was hypothesized that CSOs would report hoarding participant-driven, as well as personally-driven, motivations for engaging in accommodating behaviours, and it was hypothesized that there would be positive associations between family accommodation and 1) hoarding participant-driven motivations for engaging in accommodating behaviours, 2) personally-driven motivations for engaging in accommodating behaviours, 3 ) CSOs' own hoarding symptoms, and 4) CSOs' own OCD symptoms.

The frequency of family accommodation in hoarding was evaluated by assessing the number of CSOs who endorsed at least some accommodation of hoarding symptoms. Overall, 51 of the 52 CSOs endorsed at least some accommodating behaviours, and total accommodation scores ranged from 0 to $67(M=22.94, S D=16.46)$. The severity of family accommodation was 
explored by examining the range and distribution of FAI-H scores. The frequency and level of distress/interference that CSOs endorsed for each accommodating behaviour, as well as the standardized skewness and kurtosis scores for each FAI-H item are presented in Table 3. Overall, frequency subscale scores ranged from 0 to $31(M=11.83, S D=7.58)$ and distress/interference subscale scores ranged from 0 to $36(M=11.12, S D=9.38)$. FAI-H total scores and frequency and distress/interference subscale scores were significantly positively skewed; there were no significant violations with regards to kurtosis for the total score or subscale scores (see Table 2). The means and standard deviations for distress and interference associated with accommodating behaviours that were endorsed at least once or twice per month by CSOs are presented in Table 6 . When CSOs engaged in an accommodating behaviour at least once or twice during the past month, they endorsed at least a moderate degree of distress or interference, on average, for seven of the 11 accommodating behaviours that were assessed in the present study. Accommodation, as assessed with the FAI-H, did not vary by age ( $r \mathrm{~s}=-.06$ to $.01, p \mathrm{~s}=.68$ to .97$)$, gender ( $U \mathrm{~s}=$ 257.50 to $281.50, p s=.14$ to .30 ), ethnicity (i.e., Caucasian versus other; $U s=287.50$ to 296.50 , $p s=.85$ to .99$)$, level of employment $(U \mathrm{~s}=302.00$ to $323.50, p s=.56$ to .85$)$, or income $(r \mathrm{~s}=-$ .13 to $-.04, p s=.36$ to .81$)$.

Given that the FAI-H total and subscale scores violated the assumption of normality for parametric tests, Mann-Whitney $U$ tests were conducted to assess the extent to which FAI-H total and subscale scores differed for intimate partners of hoarding participants versus other types of dyads, as well as CSOs who lived with the hoarding participant versus CSOs who did not live with the hoarding participant. Intimate partners of hoarding participants engaged in accommodating behaviours as frequently as other types of dyads, and endorsed similar levels of distress/interference as other types of dyads (see Table 7). When living arrangement was 
Table 6

Means and Standard Deviations for Distress and Interference Associated with Accommodating Behaviours that were Endorsed at Least Once or Twice per Month by Close Significant Others

\begin{tabular}{|c|c|c|c|}
\hline & $n$ & $M$ & $S D$ \\
\hline 1. Provide reassurance & 21 & 1.19 & 1.21 \\
\hline 2. Wait for the hoarding participant & 36 & 2.44 & 1.18 \\
\hline $\begin{array}{l}\text { 3. Participate in/facilitate the hoarding participant's } \\
\text { acquiring }\end{array}$ & 26 & 1.88 & 1.34 \\
\hline $\begin{array}{l}\text { 4. Participate in/facilitate the saving of personal } \\
\text { possessions }\end{array}$ & 34 & 1.79 & 1.45 \\
\hline 5. Facilitate avoidance & 7 & 1.00 & 1.15 \\
\hline 6. Tolerate odd behaviours or household disruption & 37 & 2.35 & 1.03 \\
\hline 7. Refrain from saying/doing things & 34 & 2.03 & 1.38 \\
\hline $\begin{array}{l}\text { 8. Make excuses for the hoarding participant's } \\
\text { behaviour }\end{array}$ & 15 & 2.20 & 1.37 \\
\hline $\begin{array}{l}\text { 9. Help the hoarding participant with tasks of daily } \\
\text { living or simple decisions }\end{array}$ & 24 & 2.00 & 1.25 \\
\hline $\begin{array}{l}\text { 10. Take on the hoarding participant's } \\
\text { responsibilities }\end{array}$ & 16 & 2.38 & 1.50 \\
\hline 11. Modify your personal or family routine & 35 & 2.09 & 1.27 \\
\hline
\end{tabular}


Table 7

Mann-Whitney U Tests Comparing Family Accommodation Scores for Intimate Partners and Other Types of Dyads $(n=52)$

\begin{tabular}{|c|c|c|c|c|c|c|c|}
\hline & \multicolumn{2}{|c|}{ Intimate partner $(n=29)$} & \multicolumn{2}{|c|}{ Other type of dyad $(n=23)$} & \multirow[b]{2}{*}{$U$} & \multirow[b]{2}{*}{$p$} & \multirow[b]{2}{*}{$r$} \\
\hline & $M(S D)$ & $M d n$ & $M(S D)$ & $M d n$ & & & \\
\hline FAI-H total & $25.20(16.70)$ & 23.00 & $20.08(16.06)$ & 13.00 & 259.50 & .17 & -.19 \\
\hline FAI-H frequency & $12.72(7.87)$ & 12.00 & $10.70(7.21)$ & 8.00 & 277.50 & .30 & -.14 \\
\hline FAI-H distress/impairment & $12.48(9.09)$ & 11.00 & $9.39(9.66)$ & 5.00 & 249.00 & .12 & -.22 \\
\hline
\end{tabular}

Note. FAI-H = Family Accommodation Interview for Hoarding. 
compared, CSOs who lived with the hoarding participant engaged in accommodating behaviours more frequently than CSOs who did not live with the hoarding participant; however, CSOs who lived with the hoarding participant experienced similar levels of distress/interference, when compared with CSOs who did not live with the hoarding participant (see Table 8).

Pearson correlations were computed between family accommodation and CSOs' own hoarding and OCD symptoms to assess the extent to which these symptoms were associated with CSOs' level of accommodation (see Table 9). No significant associations were observed between FAI-H total and subscale scores and CSO-rated HRS-SR scores. Significant positive associations were found between the FAI-H total and frequency subscale scores and the total, ordering, and checking subscale scores of the CSO-rated OCI-R. Significant positive associations were found between the distress/interference scores of the FAI-H and the ordering and checking subscale scores of the CSO-rated OCI-R.

Next, CSOs' motivations for engaging in accommodating behaviours were assessed. The extent to which CSOs endorsed the five hoarding participant-driven and five personally-driven motivations are reported in Table 10. Overall, CSOs reported both hoarding participant-driven and personally-driven motivations for engaging in accommodating behaviours. The majority of CSOs endorsed the following hoarding participant-driven motivations to at least a moderate degree: make the hoarding participant's life easier, reduce the hoarding participant's distress, and improve the hoarding participant's functioning. The majority of CSOs endorsed the following personally-driven motivations to at least a moderate degree: enjoy supporting the hoarding participant, make my own life easier, avoid arguments with the hoarding participant, and reduce personal distress. Significant positive associations were observed between personally-driven motivations and FAI-H total and distress/interference subscale scores. No significant 
Table 8

Mann-Whitney U Tests Comparing Family Accommodation Scores for Close Significant Others who Live with the Hoarding Participant and Close Significant Others who do not Live with the Hoarding Participant $(n=52)$

\begin{tabular}{|c|c|c|c|c|c|c|c|}
\hline & \multicolumn{2}{|c|}{$\begin{array}{l}\text { Live together } \\
\quad(n=37)\end{array}$} & \multicolumn{2}{|c|}{$\begin{array}{l}\text { Do not live together } \\
\quad(n=15)\end{array}$} & \multirow[b]{2}{*}{$U$} & \multirow[b]{2}{*}{$p$} & \multirow[b]{2}{*}{$r$} \\
\hline & $M(S D)$ & $M d n$ & $M(S D)$ & $M d n$ & & & \\
\hline FAI-H total & $25.51(17.23)$ & 21.00 & $16.60(12.80)$ & 14.00 & 188.50 & .07 & -.25 \\
\hline FAI-H frequency & $13.30(7.94)$ & 12.00 & $8.20(5.25)$ & 7.00 & 169.50 & .03 & -.30 \\
\hline FAI-H distress/impairment & $12.22(9.63)$ & 11.00 & $8.40(8.44)$ & 8.00 & 203.00 & .13 & -.21 \\
\hline
\end{tabular}

Note. FAI-H = Family Accommodation Interview for Hoarding. 
Table 9

Association between Family Accommodation and Close Significant Others' Hoarding and Obsessive-Compulsive Disorder Symptoms

\begin{tabular}{|c|c|c|c|c|c|c|c|c|c|c|c|}
\hline Measure & 1 & 2 & 3 & 4 & 5 & 6 & 7 & 8 & 9 & 10 & 11 \\
\hline \multicolumn{12}{|l|}{ Family accommodation } \\
\hline 1. FAI-H total & - & & & & & & & & & & \\
\hline 2. FAI-H frequency & $.96^{* * *}$ & - & & & & & & & & & \\
\hline 3. FAI-H distress/interference & $.98^{* * *}$ & $.88^{* * *}$ & - & & & & & & & & \\
\hline \multicolumn{12}{|l|}{ CSO-rated hoarding symptoms } \\
\hline 4. HRS-SR & -.16 & -.10 & -.19 & - & & & & & & & \\
\hline \multicolumn{12}{|l|}{ CSO-rated OCD symptoms } \\
\hline 5. OCI-R total & $.29^{*}$ & $.28^{*}$ & $.28^{\dagger}$ & .19 & - & & & & & & \\
\hline 6. OCI-R washing & $.23^{\dagger}$ & $.26^{\dagger}$ & .20 & -.03 & $.77^{* * *}$ & - & & & & & \\
\hline 7. OCI-R obsessing & .09 & .12 & .06 & $.23^{\dagger}$ & $.77^{* * *}$ & $.43^{* *}$ & - & & & & \\
\hline 8. OCI-R hoarding & .00 & .04 & -.03 & $.45^{* *}$ & $.73^{* * *}$ & $.42^{* *}$ & $.63^{* * *}$ & - & & & \\
\hline 9. OCI-R ordering & $.36^{*}$ & $.28^{*}$ & $.40^{* *}$ & .13 & $.78^{* * *}$ & $.53^{* * *}$ & $.53^{* * *}$ & $.42^{* *}$ & - & & \\
\hline 10. OCI-R checking & $.35^{*}$ & $.34^{*}$ & $.34^{*}$ & .12 & $.81^{* * *}$ & $.58^{* * *}$ & $.47^{* *}$ & $.54^{* * *}$ & $.51^{* * *}$ & - & \\
\hline 11. OCI-R neutralising & $.25^{\dagger}$ & $.24^{\dagger}$ & $.24^{\dagger}$ & .07 & $.87^{* * *}$ & $.66^{* * *}$ & $.60^{* * *}$ & $.59^{* * *}$ & $.55^{* * *}$ & $.78^{* * * *}$ & - \\
\hline
\end{tabular}

Notes. $n \mathrm{~s}=50-52 . \mathrm{CSO}=$ Close significant other. $\mathrm{OCD}=$ Obsessive-compulsive disorder. FAI-H = Family Accommodation Interview for Hoarding. HRS-SR = Hoarding Rating Scale-Self-Report (Tolin, Frost, et al., 2010). OCI-R = Obsessive-Compulsive Inventory-

Revised (Foa et al., 2004). ${ }^{\dagger} p<.10 .{ }^{*} p<.05 .{ }^{* *} p<.01 .^{* * *} p<.001$. 
Table 10

Means and Standard Deviations for the Extent to Which Close Significant Others Endorsed Each Motivation or Attitude, and the Number and Percentage of Participants who Endorsed the Motivation or Attitude to at Least a Moderate Degree $(n=51)$

$M(S D)$

Number of participants who endorsed to at least a moderate

degree $(\%)$

Hoarding participant-driven motivations

1. Reduce hoarding behaviours

$1.33(1.58)$

$1.56(1.49)$

$2.25(1.64)$

$2.63(1.18)$

$1.51(1.60)$

5. Reduce the time the hoarding participant spends engaging in hoarding behaviours

Personally-driven motivations

1. Makes own life easier

2. Reduce personal distress

3. Enjoy supporting your close significant other

4. Avoid arguments with your close significant other

5. Enjoy engaging in the behaviours

Attitudes about engaging in accommodating behaviours

1. The accommodating behaviours are reasonable

2. The accommodating behaviours are helpful for the hoarding participant

3. The accommodating behaviours are helpful for the CSO

4. The accommodating behaviours need to be decreased

5. The accommodating behaviours need to be increased

36 (70.59)

1.69 (1.62)

26 (50.98)

2.35 (1.45)

37 (72.55)

2.41 (1.69)

35 (68.63)

0.76 (1.24)

13 (25.49)

$2.78(1.15)$

$46(90.20)$

2.50 (1.14)

44 (86.27)

$2.08(1.42)$

35 (68.63)

1.06 (1.33)

$16(31.37)$

0.83 (1.26)

13 (25.49) 
associations were found between hoarding participant-driven motivations and FAI-H total or subscale scores (see Table 11).

Lastly, CSOs' attitudes about engaging in accommodating behaviours were assessed. The extent to which CSOs endorsed various attitudes about accommodating behaviours is presented in Table 9. Most CSOs believed, at least to a moderate degree, that their accommodating behaviours were reasonable. In addition, the majority of CSOs also believed, at least to a moderate degree, that their behaviours were helpful for the hoarding participant and less so, for themselves. Few CSOs indicated that they needed to change the frequency of their accommodating behaviours (i.e., only $30 \%$ reported that they needed to decrease their behaviours, and $25 \%$ reported that they needed to increase their behaviours).

Posthoc analyses. Posthoc analyses were conducted to compare family accommodation scores for CSOs of individuals who endorsed hoarding symptoms that met diagnostic criteria for hoarding disorder $(n=36)$ with CSOs of individuals who did not endorse hoarding symptoms that met diagnostic criteria for hoarding disorder $(n=16)$. Given that FAI-H total scores and distress/interference subscale scores violated the assumption of normality for parametric tests, Mann-Whitney $U$ tests were conducted to assess the extent to which FAI-H total and subscale scores differed for these two groups. CSOs of individuals who endorsed hoarding symptoms that met diagnostic criteria for hoarding disorder reported greater total accommodation $(M=26.67$, $S D=16.96, M d n=23.50$ ) when compared with CSOs of individuals who did not endorse hoarding symptoms that met diagnostic criteria for hoarding disorder $(M=14.56, S D=11.94$, $M d n=12.50), U=159.50, p=.011, r=-.35$. In addition, CSOs of individuals who endorsed hoarding symptoms that met diagnostic criteria for hoarding disorder $(M=13.50, S D=7.71$, $M d n=12.00)$ engaged in accommodation more frequently than CSOs of individuals who did not 
Table 11

Association between Family Accommodation and Hoarding Participant-Driven and Personally-Driven Motivations

\begin{tabular}{|c|c|c|c|c|c|}
\hline Measure & 1 & 2 & 3 & 4 & 5 \\
\hline 1. FAI-H total & - & & & & \\
\hline 2. FAI-H frequency & $.96^{* * *}$ & - & & & \\
\hline 3. FAI-H distress/interference & $.98^{* * *}$ & $.88^{* * *}$ & - & & \\
\hline $\begin{array}{l}\text { 4. Hoarding participant-driven } \\
\text { motivations }\end{array}$ & .22 & $.23^{\dagger}$ & .19 & - & \\
\hline 5. Personally-driven motivations & $.29^{*}$ & $.28^{\dagger}$ & $.28^{*}$ & $.32^{*}$ & - \\
\hline
\end{tabular}


endorse hoarding symptoms that met diagnostic criteria for hoarding disorder $(M=8.06, S D=$ $5.90, M d n=8.50), U=167.00, p=.016, r=-.33$, and they also reported greater levels of distress/interference associated with accommodation $(M=13.17, S D=9.78, M d n=11.00)$, when compared with CSOs of individuals who endorsed hoarding symptoms that met diagnostic criteria for hoarding disorder $(M=6.50, S D=6.58, M d n=4.50), U=167.00, p=.016, r=-.33$.

\section{Study Aim \#3}

The third aim of the present study was to investigate the construct of family accommodation in hoarding by examining the association between CSOs' accommodating behaviours and hoarding participant- and CSO-rated hoarding symptom severity, relationship satisfaction, hoarding participant- and CSO-rated functional impairment, CSOs' attributions about the hoarding participant's control over his/her hoarding behaviours, CSOs' rejecting attitudes toward the hoarding participant, and hoarding participants' and CSOs' wellbeing. Consistent with previous research, it was hypothesized that family accommodation would be positively associated with hoarding symptom severity, functional impairment, and increased rejection toward the hoarding participant. In addition, it was also hypothesized that family accommodation would be negatively associated with relationship satisfaction, CSOs' attribution about the hoarding participant's control over their hoarding behaviour, and wellbeing.

The associations between family accommodation and hoarding symptom severity are presented in Table 12. There were significant positive correlations between FAI-H total and subscale scores and clinician-rated HRS-I scores. There was a significant positive correlation between FAI-H total scores and hoarding participant-rated SI-R difficulty discarding subscale scores. There were significant positive correlations between FAI-H frequency subscale scores and hoarding participant-rated SI-R total scores and difficulty discarding subscale scores. There 
Table 12

Association between Family Accommodation and Hoarding Symptom Severity $(n=52)$

\begin{tabular}{|c|c|c|c|c|c|c|c|c|c|c|c|c|c|c|}
\hline Measure & 1 & 2 & 3 & 4 & 5 & 6 & 7 & 8 & 9 & 10 & 11 & 12 & 13 & 14 \\
\hline \multicolumn{15}{|l|}{ Family accommodation } \\
\hline 1. FAI-H total & - & & & & & & & & & & & & & \\
\hline 2. FAI-H frequency & $.96^{* * *}$ & - & & & & & & & & & & & & \\
\hline $\begin{array}{l}\text { 3. FAI-H } \\
\text { distress/interference }\end{array}$ & $.98^{* * *}$ & $.88^{* * *}$ & - & & & & & & & & & & & \\
\hline Clinician-rated symptoms & & & & & & & & & & & & & & \\
\hline 4. HRS-I & $.50^{* * *}$ & $.51^{* * *}$ & $.47^{* * *}$ & - & & & & & & & & & & \\
\hline \multicolumn{15}{|l|}{$\begin{array}{l}\text { Hoarding participant-rated } \\
\text { symptoms }\end{array}$} \\
\hline 5. SI-R total & $.26^{\dagger}$ & $.28^{*}$ & $.23^{\dagger}$ & $.79^{* * *}$ & - & & & & & & & & & \\
\hline $\begin{array}{l}\text { 6. SI-R difficulty } \\
\text { discarding }\end{array}$ & $.29^{*}$ & $.30^{*}$ & $.26^{\dagger}$ & $.61^{* * *}$ & $.84^{* * *}$ & - & & & & & & & & \\
\hline 7. SI-R acquisition & .18 & .21 & .15 & $.65^{* * *}$ & $.84^{* * *}$ & $.64^{* * *}$ & - & & & & & & & \\
\hline 8. SI-R clutter & .22 & $.24^{\dagger}$ & .20 & $.75^{* * *}$ & $.89^{* * *}$ & $.62^{* * *}$ & $.59^{* * *}$ & - & & & & & & \\
\hline 9. CIR & .19 & .18 & .19 & $.50^{* * *}$ & $.56^{* * *}$ & $.30^{*}$ & $.41^{* *}$ & $.65^{* * *}$ & - & & & & & \\
\hline \multicolumn{15}{|l|}{ CSO-rated symptoms } \\
\hline 10. SI-R total & $.37^{* *}$ & $.32^{*}$ & $.39^{* *}$ & $.62^{* * *}$ & $.55^{* * *}$ & $.33^{*}$ & $.55^{* * *}$ & $.54^{* * *}$ & $.44^{* *}$ & - & & & & \\
\hline $\begin{array}{l}\text { 11. SI-R difficulty } \\
\text { discarding }\end{array}$ & $.41^{* *}$ & $.38^{* *}$ & $.41^{* *}$ & $.47^{* * *}$ & $.36^{* *}$ & $.23^{\dagger}$ & $.39^{* *}$ & $.32^{*}$ & $.26^{\dagger}$ & $.82^{* * *}$ & - & & & \\
\hline 12. SI-R acquisition & $.23^{\dagger}$ & .18 & $.27^{\dagger}$ & $.50^{* * *}$ & $.47^{* * *}$ & $.32^{*}$ & $.57^{* * *}$ & $.36^{* *}$ & $.29^{*}$ & $.89^{* * *}$ & $.62^{* * *}$ & - & & \\
\hline 13. SI-R clutter & $.35^{*}$ & $.30^{*}$ & $.37^{* *}$ & $.63^{* * *}$ & $.55^{* * *}$ & $.30^{*}$ & $.46^{* *}$ & $.62^{* * *}$ & $.51^{* * *}$ & $.93^{* * *}$ & $.63^{* * *}$ & $.73^{* * *}$ & - & \\
\hline 14. CIR & $.33^{*}$ & $.30^{*}$ & $.35^{*}$ & $.49^{* * *}$ & $.60^{* * *}$ & $.42^{* *}$ & $.46^{* *}$ & $.66^{* * *}$ & $.75^{* * *}$ & $.76^{* * *}$ & $.58^{* * *}$ & $.64^{* * *}$ & $.75^{* * *}$ & - \\
\hline
\end{tabular}

Notes. CSO = Close significant other. FAI-H = Family Accommodation Interview for Hoarding. HRS-I = Hoarding Rating ScaleInterview (Tolin, Frost, et al., 2010). SI-R = Saving Inventory-Revised (Frost et al., 2004). CIR = Clutter Image Rating (Frost et al., 2008). ${ }^{\dagger} p<.10 .{ }^{*} p<.05 .{ }^{* *} p<.01 .{ }^{* * *} p<.001$. 
were significant positive correlations between FAI-H total and subscale scores and CSO-rated SI-R total scores, difficulty discarding subscale scores, clutter subscale scores, and CSO-rated CIR composite scores.

The associations between family accommodation and relationship satisfaction, CSOs' rejecting attitudes toward the hoarding participants, and CSOs' attributions about the hoarding participant's control over hoarding behaviours are presented in Table 13. There were significant negative correlations between FAI-H total and subscale scores and hoarding participant-rated QRI support subscale scores, and significant positive correlations between FAI-H total and subscale scores and hoarding participant-rated QRI conflict subscale scores. There were significant positive correlations between FAI-H total and subscale scores and CSO-rated QRI conflict subscale scores and PRS scores. There was a significant negative correlation between FAI-H distress/interference subscale scores and CSO-rated QRI depth subscale scores.

Posthoc analyses. Although not a specific aim of the present study, posthoc analyses were conducted to examine the association between hoarding symptom severity and relationship variables, including relationship satisfaction and CSOs' rejecting attitudes toward the individual with the hoarding problem, for both hoarding participants and CSOs. There were significant associations between relationship conflict and CSO-rated total hoarding symptom severity, $r$ $=.45, p=.001$, and CSOs' ratings of the severity of the hoarding participants' difficulties with discarding, $r=.40, p=.003$, acquiring, $r=.47, p<.001$, and clutter, $r=.35, p=.012$. Similarly, there were significant associations between relationship conflict and hoarding participant-rated total hoarding symptom severity, $r=.40, p=.003$, and the hoarding participants' ratings of the severity of their own difficulties with discarding, $r=.37, p=.008$, acquiring, $r=.32, p=.024$, and clutter, $r=.36, p=.009$. There was a significant association between relationship support 
Table 13

Association between Family Accommodation and Relationship Satisfaction

\begin{tabular}{|c|c|c|c|c|c|c|c|c|c|c|c|}
\hline Measure & 1 & 2 & 3 & 4 & 5 & 6 & 7 & 8 & 9 & 10 & 11 \\
\hline \multicolumn{12}{|l|}{ Family accommodation } \\
\hline 1. FAI-H total & - & & & & & & & & & & \\
\hline 2. FAI-H frequency & $.96^{* * *}$ & - & & & & & & & & & \\
\hline 3. FAI-H distress/interference & $.98^{* * *}$ & $.88^{* * *}$ & - & & & & & & & & \\
\hline \multicolumn{12}{|l|}{$\begin{array}{l}\text { Hoarding participant relationship } \\
\text { satisfaction }\end{array}$} \\
\hline 4. QRI support & $-.34^{*}$ & $-.34^{*}$ & $-.32^{*}$ & - & & & & & & & \\
\hline 5. QRI conflict & $.34^{*}$ & $.38^{* *}$ & $.29^{*}$ & $-.44^{* *}$ & - & & & & & & \\
\hline 6. QRI depth & -.22 & -.21 & -.21 & $.66^{* * *}$ & -.19 & - & & & & & \\
\hline \multicolumn{12}{|l|}{ CSO relationship satisfaction } \\
\hline 7. QRI support & -.21 & -.13 & $-.26^{\dagger}$ & $.40^{* *}$ & -.18 & $.36^{* *}$ & - & & & & \\
\hline 8. QRI conflict & $.62^{* * *}$ & $.58^{* * *}$ & $.62^{* * *}$ & $-.48^{* * *}$ & $.58^{* * *}$ & $-.26^{\dagger}$ & $-.26^{\dagger}$ & - & & & \\
\hline 9. QRI depth & $-.26^{\dagger}$ & -.18 & $-.31^{*}$ & $.36^{* *}$ & -.14 & $.38^{* *}$ & $.78^{* * *}$ & -.16 & - & & \\
\hline 10. PRS & $.51^{* * *}$ & $.46^{* *}$ & $.52^{* * *}$ & $-.35^{*}$ & $.30^{*}$ & $-.33^{*}$ & $-.51^{* * *}$ & $.57^{* * *}$ & $-.43^{* *}$ & - & \\
\hline 11. HAQ & -.02 & -.02 & -.02 & -.07 & .08 & -.04 & .06 & -.01 & .06 & .05 & - \\
\hline
\end{tabular}

Notes. $n \mathrm{~s}=51-52 . \mathrm{CSO}=$ Close significant other. FAI-H = Family Accommodation Interview for Hoarding. QRI $=$ Quality of Relations Inventory (Pierce et al., 1991). PRS = Patient Rejection Scale (Kreisman et al., 1979). HAQ = Hoarding Attribution Questionnaire. ${ }^{\dagger} p<.10 .{ }^{*} p<.05 .{ }^{* *} p<.01 .^{* * *} p<.001$. 
and the hoarding participants' rating of the severity of their own acquiring, $r=-.30, p=.029$. CSOs' rejecting attitudes toward the individual with the hoarding problem were significantly associated with CSO-rated total hoarding symptom severity, $r=.34, p=.013$, CSOs ratings of the severity of the hoarding participants' difficulties with discarding, $r=.28, p=.045$, and clutter, $r=.35, p=.010$. CSOs' rejecting attitudes toward the individual with the hoarding problem were not significantly associated with CSOs rating of the severity of the hoarding participants' acquiring, $r=.25, p=.075$, as well as hoarding participant-rated total hoarding symptom severity, $r=.09, p=.508$, or the hoarding participants' ratings of the severity of their own difficulties with discarding, $r=.12, p=.381$, acquiring, $r=.03, p=.848$, and clutter, $r$ $=.09, p=.509$.

The associations between family accommodation and functional impairment are presented in Table 14. Although most of the correlations between family accommodation and the various domains of functional impairment were nonsignificant, there were significant positive correlations between FAI-H distress/interference subscale scores and CSO-rated IIRS total scores and ADL-H scores. There were also significant positive correlations between FAI-H total and subscale scores and CSO-rated IIRS relationship subscale scores, and between FAI-H distress/interference subscale scores and hoarding participant-rated IIRS relationship subscale scores.

The associations between family accommodation and wellbeing are presented in Table 15 . There was a significant positive correlation between FAI-H frequency subscale scores and hoarding participant-rated AQ anger subscale scores. There were no significant correlations between FAI-H total and subscale scores and any measures of CSO-rated wellbeing. 
Table 14

Association between Family Accommodation and Functional Impairment

\begin{tabular}{|c|c|c|c|c|c|c|c|c|c|c|c|c|c|}
\hline Measure & 1 & 2 & 3 & 4 & 5 & 6 & 7 & 8 & 9 & 10 & 11 & 12 & 13 \\
\hline \multicolumn{14}{|l|}{ Family accommodation } \\
\hline 1. FAI-H total & - & & & & & & & & & & & & \\
\hline 2. FAI-H frequency & $.96^{* * *}$ & - & & & & & & & & & & & \\
\hline 3. FAI-H distress/interference & $.98^{* * *}$ & $.88^{* * *}$ & - & & & & & & & & & & \\
\hline \multicolumn{14}{|l|}{$\begin{array}{l}\text { Hoarding participant-rated } \\
\text { impairment }\end{array}$} \\
\hline 4. IIRS total & .10 & .13 & .06 & - & & & & & & & & & \\
\hline 5. IIRS lifestyle & .10 & .14 & .05 & $.92^{* * *}$ & - & & & & & & & & \\
\hline 6. IIRS activity & -.02 & .04 & -.06 & $.94^{* * *}$ & $.80^{* * * *}$ & - & & & & & & & \\
\hline 7. IIRS relationships & $.29^{\dagger}$ & .24 & $.31^{*}$ & $.66^{* * *}$ & $.43^{* *}$ & $.51^{* * *}$ & - & & & & & & \\
\hline 8. ADL-H & .20 & $.24^{\dagger}$ & .15 & $.51^{* * *}$ & $.48^{* * * *}$ & $.50^{* * *}$ & .24 & - & & & & & \\
\hline \multicolumn{14}{|l|}{ CSO-rated impairment } \\
\hline 9. IIRS total & $.26^{\dagger}$ & .19 & $.30^{*}$ & $.38^{* *}$ & $.32^{*}$ & $.34^{*}$ & .24 & $.35^{*}$ & - & & & & \\
\hline 10. IIRS lifestyle & .17 & .16 & .17 & $.41^{* *}$ & $.38^{* *}$ & $.38^{* *}$ & .18 & $.48^{* *}$ & $.90^{* * *}$ & - & & & \\
\hline 11. IIRS activity & .21 & .13 & $.27^{\dagger}$ & $.32^{*}$ & $.24^{\dagger}$ & $.31^{*}$ & .22 & $.35^{*}$ & $.94^{* * *}$ & $.80^{* * * *}$ & - & & \\
\hline 12. IIRS relationships & $.38^{*}$ & $.32^{*}$ & $.41^{* *}$ & .20 & .15 & .16 & .21 & .11 & $.73^{* * *}$ & $.51^{* * *}$ & $.56^{* * *}$ & - & \\
\hline 13. ADL-H & $.26^{\dagger}$ & .19 & $.31^{*}$ & .07 & .01 & .07 & .11 & $.28^{*}$ & $.37^{* *}$ & $.35^{*}$ & $.42^{* *}$ & .22 & - \\
\hline
\end{tabular}

Notes. $n \mathrm{~s}=45-52 . \mathrm{CSO}=$ Close significant other. FAI-H = Family Accommodation Interview for Hoarding. IIRS = Illness Intrusiveness Ratings Scale (Devins, 1994). ADL-H = Activities of Daily Living in Hoarding Scale (Frost et al., in press). ${ }^{\dagger} p<.10$. ${ }^{*} p<.05 .{ }^{* *} p<.01 .{ }^{* * *} p<.001$. 
Table 15

Association between Family Accommodation and Wellbeing

\begin{tabular}{|c|c|c|c|c|c|c|c|c|c|c|c|c|c|}
\hline Measure & 1 & 2 & 3 & 4 & 5 & 6 & 7 & 8 & 9 & 10 & 11 & 12 & 13 \\
\hline \multicolumn{14}{|l|}{ Family accommodation } \\
\hline 1. FAI-H total & - & & & & & & & & & & & & \\
\hline 2. FAI-H frequency & $.96^{* * *}$ & - & & & & & & & & & & & \\
\hline $\begin{array}{l}\text { 3. FAI-H } \\
\text { distress/interference }\end{array}$ & $.98^{* * *}$ & $.88^{* * *}$ & - & & & & & & & & & & \\
\hline \multicolumn{14}{|l|}{$\begin{array}{l}\text { Hoarding participant-rated } \\
\text { wellbeing }\end{array}$} \\
\hline 4. DASS-21 depression & -.03 & -.07 & -.00 & - & & & & & & & & & \\
\hline 5. DASS-21 anxiety & .15 & .18 & .12 & $.66^{* * *}$ & - & & & & & & & & \\
\hline 6. DASS-21 stress & .17 & .20 & .13 & $.53^{* * *}$ & $.69^{* * *}$ & - & & & & & & & \\
\hline 7. AQ anger & $.26^{\dagger}$ & $.29^{*}$ & .22 & .14 & .13 & $.45^{* *}$ & - & & & & & & \\
\hline 8. AQ hostility & .04 & .05 & .03 & $.51^{* * *}$ & $.33^{*}$ & $.44^{* *}$ & $.44^{* *}$ & - & & & & & \\
\hline \multicolumn{14}{|l|}{ CSO-rated wellbeing } \\
\hline 9. DASS-21 depression & .10 & .12 & .07 & -.11 & -.02 & -.04 & -.12 & .02 & - & & & & \\
\hline 10. DASS-21 anxiety & .22 & .22 & .20 & -.12 & .05 & .02 & .04 & -.03 & $.78^{* * *}$ & - & & & \\
\hline 11. DASS-21 stress & .21 & .22 & .20 & -.12 & .00 & -.04 & -.13 & -.12 & $.79^{* * *}$ & $.79^{* * *}$ & - & & \\
\hline 12. AQ anger & -.07 & -.05 & -.08 & -.10 & -.18 & -.05 & .05 & .04 & $.26^{\dagger}$ & .19 & $.28^{*}$ & - & \\
\hline 13. AQ hostility & -.01 & -.02 & -.00 & -.14 & -.17 & -.14 & -.09 & .01 & $.55^{* * *}$ & $.41^{* *}$ & $.54^{* * *}$ & $.44^{* *}$ & - \\
\hline
\end{tabular}

Notes. $n \mathrm{~s}=51-52 . \mathrm{CSO}=$ Close significant other. FAI-H = Family Accommodation Interview for Hoarding. DASS-21 = Depression Anxiety Stress Scales-21 item version (Lovibond \& Lovibond, 1995). AQ = Aggression Questionnaire-15-item short form (Buss \& Warren, 2000). ${ }^{\dagger} p<.10 .{ }^{*} p<.05 .{ }^{* *} p<.01 .{ }^{* * *} p<.001$. 
Posthoc analyses. Given the significant positive association that was found between FAI$\mathrm{H}$ frequency subscale scores and hoarding participant-rated AQ anger subscale scores, posthoc analyses were conducted to assess the extent to which CSOs may have accommodated hoarding participants hoarding symptoms to prevent arguments and reduce hoarding participants' distress. This hypothesis was tested by examining the associations between the frequency of CSOs' accommodating behaviours and CSOs' responses to individual motivation items that were assessed as part of study aim \#2 (i.e., item 3, reduce the hoarding participant's distress, and item 9, avoid arguments with the hoarding participant). The frequency of CSOs' accommodating behaviours was positively and significantly associated with motivation item 3 (reduce the hoarding participant's distress), $r=.31, p=.03$, and motivation item 9 (avoid arguments with the hoarding participant), $r=.46, p=.001$.

\section{Study Aim \#4}

The fourth aim of the study was to investigate the extent to which family accommodation mediated the association between hoarding symptom severity and relationship satisfaction, as well as the association between hoarding symptom severity and functional impairment.

Consistent with previous research, it was hypothesized that family accommodation would mediate the association between hoarding symptom severity and relationship satisfaction, as well as the association between hoarding symptom severity and functional impairment.

Pearson correlations were computed to assess the extent to which hoarding participantand CSO-rated hoarding symptom severity, family accommodation, relationship satisfaction, and functional impairment were nonindependent. It is typically recommended that the test for nonindependence use a two-tailed alpha of .20 (Myers, 1979, as cited in Kenny et al., 2006). Hoarding participants' and CSOs' scores were found to be nonindependent for all variables that 
were included in the dyadic data analyses in the present study. There was a significant positive association between hoarding participant- and CSO-rated hoarding symptom severity, $r=.55, p$ $<.001$. There were significant positive associations between hoarding participant- and CSOrated total family accommodation, $r=.49, p<.001$, frequency of family accommodation, $r=.54$, $p<.001$, and distress/interference associated with family accommodation, $r=.42, p=.002$. There were significant positive associations between hoarding participant- and CSO-rated relationship support, $r=.40, p=.004$, relationship conflict, $r=.58, p<.001$, and relationship depth, $r=.38, p=.006$. There was a significant positive association between hoarding participant- and CSO-rated activities of daily living, $r=.28, p=.045$.

\section{Relationship satisfaction.}

\section{Examining the association between total hoarding symptom severity and relationship}

support. An interaction model was tested to examine the extent to which the actor and partner effects for total hoarding symptom severity were significantly associated with relationship support, and whether these associations were moderated by participant status (i.e., hoarding participant versus CSO). The main effects of actor total hoarding symptom severity, partner total hoarding symptom severity, participant status, as well as the interaction terms between participant status and actor total hoarding symptom severity, and participant status and partner total hoarding symptom severity were entered as fixed effects. The results of this interaction model are presented in Table 16.

As displayed in Table 16, there were no significant actor or partner effects, or moderating influence of participant status. Given that participant status did not moderate the association between total hoarding symptom severity and relationship support, it was not necessary to test a two-intercept model to determine the extent to which the actor and partner effects differed for 
Table 16

Interaction Model Estimating Effects of Overall Hoarding Symptom Severity on Relationship Support $(n=52)$

\begin{tabular}{lccc}
\hline & $b(S E)$ & $t$ & $p$ \\
\hline Participant status & $0.200(0.200)$ & 1.01 & .319 \\
Actor hoarding symptoms & $-0.007(0.004)$ & -1.79 & .077 \\
Partner hoarding symptoms & $-0.001(0.004)$ & -0.19 & .853 \\
Participant status*actor hoarding symptoms & $0.000(0.005)$ & 0.006 & .995 \\
Participant status*partner hoarding symptoms & $-0.003(0.005)$ & -0.661 & .511
\end{tabular}

Notes. Pseudo $R^{2}=.004$. Participant status $=$ Status of the participant (hoarding participant versus close significant other). Actor hoarding symptoms $=$ Actor overall hoarding symptom severity, as assessed with the Saving Inventory-Revised (Frost et al., 2004) total score. Partner hoarding symptoms = Partner overall hoarding symptoms, as assessed with the Saving Inventory-Revised (Frost et al., 2004) total score. 
hoarding participants versus CSOs. Moreover, given that there were no significant actor or partner effects, total family accommodation was not investigated as a potential mediator between hoarding symptom severity and relationship support.

Examining the association between total hoarding symptom severity and relationship

conflict. An interaction model was tested to determine the extent to which the actor and partner effects for total hoarding symptom severity were significantly associated with relationship conflict, and whether these associations were moderated by participant status (i.e., hoarding participant versus CSO). The main effects of actor total hoarding symptom severity, partner total hoarding symptom severity, participant status, as well as the interaction terms between participant status and actor total hoarding symptom severity, and participant status and partner total hoarding symptom severity were entered as fixed effects. The results of this interaction model are presented in Table 17.

As displayed in Table 17, there was a significant actor effect, such that actor total hoarding symptom severity was positively associated with actor relationship conflict. There was no significant partner effect, or moderating influence of participant status. Given that participant status did not moderate the association between total hoarding symptom severity and relationship conflict, it was not necessary to test a two-intercept model to determine the extent to which the actor and partner effects differed for hoarding participants versus CSOs.

Examining total family accommodation as a mediator between total hoarding symptom severity and relationship conflict. Given that participant status did not moderate the association between total hoarding symptom severity and relationship conflict (see Table 17), the meditational effects of total family accommodation were averaged across hoarding participants and CSOs. Participant status was entered as a fixed effect in each step. 
Table 17

Interaction Model Estimating Effects of Overall Hoarding Symptom Severity on Relationship Conflict $(n=51)$

\begin{tabular}{lcrr}
\hline & $b(S E)$ & $t$ & .839 \\
\hline Participant status & $-0.029(0.143)$ & -0.20 & $<.001$ \\
Actor hoarding symptoms & $0.014(0.003)$ & 4.29 & .475 \\
Partner hoarding symptoms & $0.002(0.003)$ & 0.72 & .580 \\
Participant status*actor hoarding symptoms & $-0.002(0.004)$ & -0.56 & .682 \\
Participant status*partner hoarding symptoms & $0.002(0.004)$ & 0.41 &. .
\end{tabular}

Notes. Pseudo $R^{2}=.173$. Participant status = Status of the participant (hoarding participant versus close significant other). Actor hoarding symptoms $=$ Actor overall hoarding symptom severity, as assessed with the Saving Inventory-Revised (Frost et al., 2008) total score. Partner hoarding symptoms = Partner overall hoarding symptoms, as assessed with the Saving Inventory-Revised (Frost et al., 2008) total score. 
Results of the APIMeM are displayed in Figure 4. In the first step of mediation, which tests the associations between actor and partner total hoarding symptom severity and actor total family accommodation (path a), there was a significant actor effect. Total actor hoarding symptom severity was positively associated with total actor family accommodation. The partner effect was not significant. In the second step of mediation, which tests the associations between actor and partner total family accommodation and actor relationship conflict (path b), there was a significant actor effect. Total actor family accommodation was positively associated with actor relationship conflict. There was also a significant partner effect, such that total partner family accommodation was positively associated with actor relationship conflict. The third step of mediation, which tests the associations between actor and partner total hoarding symptom severity and actor relationship conflict (path c), is analogous with the interaction model that was presented in Table 17. As previously mentioned, there was a significant actor effect between total hoarding symptom severity and relationship conflict, such that total actor hoarding symptom severity was positively associated with actor relationship conflict. There was no significant partner effect. In the final step of mediation, which tests the associations between actor and partner total hoarding symptom severity and actor relationship conflict after accounting for total family accommodation (path c'), the actor effect between total hoarding symptom severity and relationship conflict was reduced, but still significant, after accounting for total family accommodation. The partner effect remained nonsignificant after accounting for total family accommodation. Results of the Sobel test indicate that total family accommodation partially mediated the actor effect between total hoarding symptom severity and relationship conflict (see Table 18). The positive association between actor total hoarding symptom severity and actor relationship conflict was significantly decreased by actor total family accommodation. 


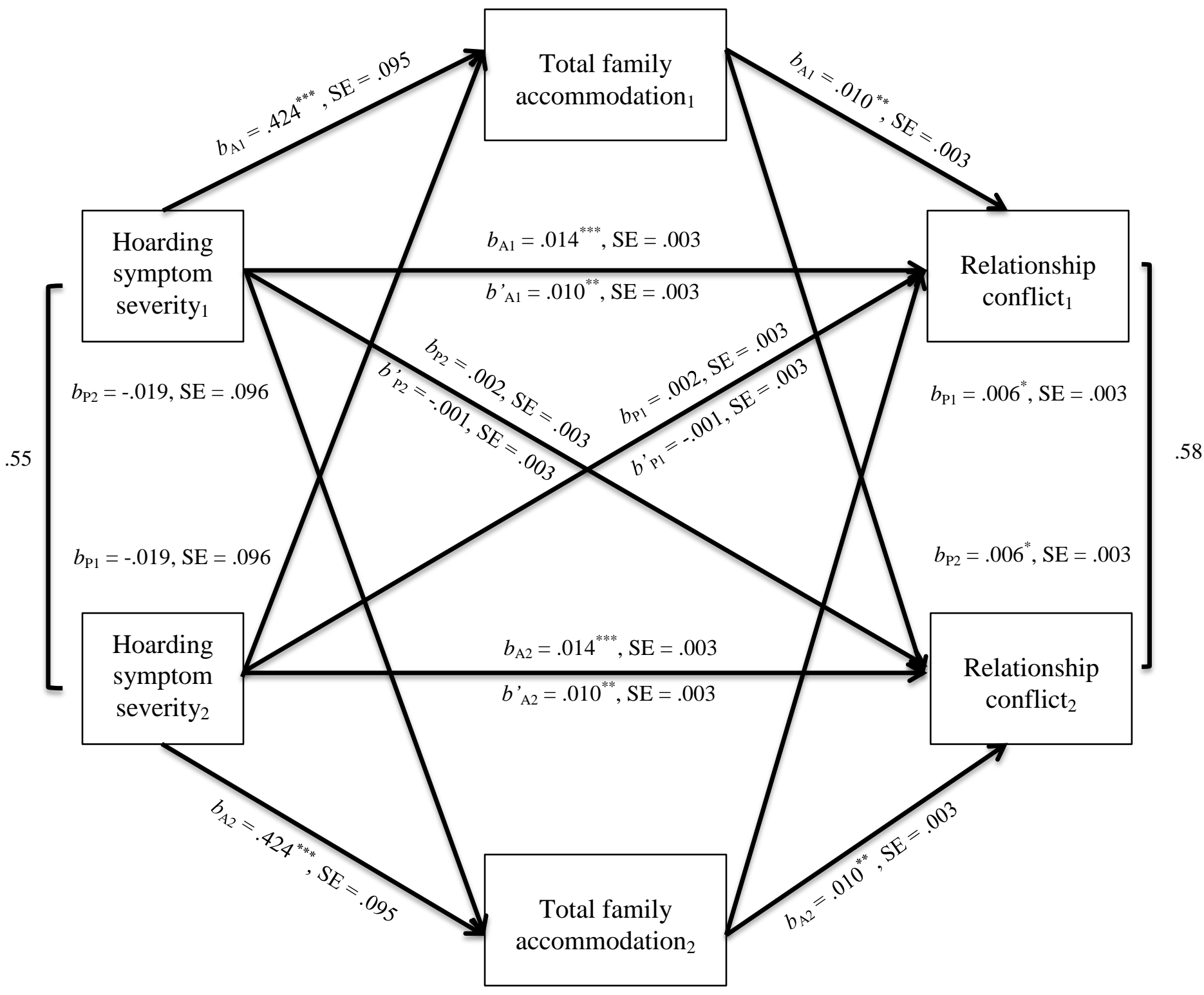

Figure 4. Actor-partner interdependence mediation model (APIMeM) assessing total family accommodation as a mediator of the association between overall hoarding symptom severity and relationship conflict, averaging across dyads. ${ }_{A}=$ Actor effect. $P=$ Partner effect. ${ }_{1}=$ Dyad member 1. ${ }_{2}=$ Dyad member $2{ }^{*} * p<.01 . * * * p<.001$. 
Table 18

Sobel Test Assessing Total Family Accommodation as a Mediator of the Association between Overall Hoarding Severity and Relationship Conflict $(n=51)$

\begin{tabular}{llccc}
\hline & Indirect effects & $\mathrm{SE}$ & $\mathrm{Z}$ & $p$ \\
\hline Actor-actor & $\mathrm{H}_{1} \rightarrow \mathrm{A}_{1} \rightarrow \mathrm{C}_{1}$ & 0.002 & 2.67 & .008 \\
Partner-partner & $\mathrm{H}_{1} \rightarrow \mathrm{A}_{2} \rightarrow \mathrm{C}_{1}$ & 0.001 & -0.20 & .844 \\
Actor-partner & $\mathrm{H}_{2} \rightarrow \mathrm{A}_{2} \rightarrow \mathrm{C}_{1}$ & 0.001 & 1.83 & .068 \\
Partner-actor & $\mathrm{H}_{2} \rightarrow \mathrm{A}_{1} \rightarrow \mathrm{C}_{1}$ & 0.001 & -0.20 & .843 \\
\hline
\end{tabular}

Notes. $\mathrm{H}$ (predictor variable) = Overall hoarding symptom severity, as assessed with the Saving Inventory-Revised (Frost et al., 2008) total score. A (mediator) = Total family accommodation, as assessed with the Family Accommodation Interview for Hoarding total score. C (outcome variable) $=$ Relationship support, as assessed with the Quality of Relations Inventory (Pierce et al., 1991) conflict subscale. $1=$ Dyad member $1.2=$ Dyad member 2. 
Approximately $29 \%$ of the actor effect was partially mediated by actor total family accommodation. $^{1}$

\section{Examining the association between total hoarding symptom severity and relationship}

depth. An interaction model was conducted to determine the extent to which the actor and partner effects for total hoarding symptom severity were significantly associated with relationship depth, and whether these associations were moderated by participant status (i.e., hoarding participant versus CSO). The main effects of actor total hoarding symptom severity, partner total hoarding symptom severity, participant status, as well as the interaction terms between participant status and actor total hoarding symptom severity, and participant status and partner total hoarding symptom severity were entered as fixed effects. The results of this interaction model are presented in Table 19.

As displayed in Table 19, there were no significant actor or partner effects, or moderating influence of participant status. Given that participant status did not moderate the association between total hoarding symptom severity and relationship depth, it was not necessary to test a two-intercept model to determine the extent to which the actor and partner effects differed for hoarding participants versus CSOs. Moreover, given that there were no significant actor or partner effects, total family accommodation was not investigated as a potential mediator between total hoarding symptom severity and relationship depth.

\footnotetext{
${ }^{1}$ Similar results were found when the distress/interference subscale of the FAI-H was entered as a mediator of the association between total hoarding symptom severity and relationship conflict. Specifically, actor distress/interference partially mediated the actor effect between total hoarding symptom severity and relationship conflict. The frequency subscale of the FAI-H, however, was not a significant mediator of the association between total hoarding symptom severity and relationship conflict.
} 
Table 19

Interaction Model Estimating Effects of Overall Hoarding Symptom Severity on Relationship Depth $(n=51)$

\begin{tabular}{lccc}
\hline & $b(S E)$ & $t$ & .756 \\
\hline Participant status & $0.051(0.165)$ & 0.31 & .348 \\
Actor hoarding symptoms & $-0.003(0.003)$ & -0.94 & .22 \\
Partner hoarding symptoms & $0.001(0.003)$ & 1.00 & .828 \\
Participant status*actor hoarding symptoms & $0.004(0.004)$ & -1.10 & .321 \\
Participant status*partner hoarding symptoms & $-0.004(0.004)$ & .276
\end{tabular}

Notes. Pseudo $R^{2}=0$. Participant status = Status of the participant (hoarding participant versus close significant other). Actor hoarding symptoms $=$ Actor overall hoarding symptom severity, as assessed with the Saving Inventory-Revised (Frost et al., 2008) total score. Partner hoarding symptoms = Partner overall hoarding symptoms, as assessed with the Saving Inventory-Revised (Frost et al., 2008) total score. 


\section{Functional impairment.}

\section{Examining the association between total hoarding symptom severity and activities of}

daily living. An interaction model was conducted to determine the extent to which the actor and partner effects for total hoarding symptom severity were significantly associated with activities of daily living, and whether these associations were moderated by participant status (i.e., hoarding participant versus CSO). The main effects of actor total hoarding symptom severity, partner total hoarding symptom severity, participant status, as well as the interaction terms between participant status and actor total hoarding symptom severity, and participant status and partner total hoarding symptom severity were entered as fixed effects. The results of this interaction model are presented in Table 20.

As displayed in Table 20, there was a significant actor effect, such that total actor hoarding symptom severity was positively associated with impairment in activities of daily living. In addition, there was a significant interaction between participant status and total actor hoarding symptom severity, suggesting that the magnitude of the actor effect differed when hoarding participants were compared with CSOs.

Given that participant status moderated the association between total hoarding symptom severity and activities of daily living, a two-intercept model was conducted to determine the extent to which the actor and partner effects differed for hoarding participants and CSOs. The results of this two-intercept model are presented in Table 21. As displayed in Table 21, there were significant actor effects for both hoarding participants and CSOs, such that total hoarding symptom severity was positively associated with impairment in activities of daily living for both hoarding participants and CSOs. There was also a significant partner effect for hoarding 
Table 20

Interaction Model Estimating Effects of Overall Hoarding Symptom Severity on Activities of Daily Living $(n=51)$

\begin{tabular}{lcrr}
\hline & $b(S E)$ & $t$ & \multicolumn{1}{c}{$t$} \\
\hline Participant status & $-0.079(0.218)$ & -0.36 & .720 \\
Actor hoarding symptoms & $0.025(0.004)$ & 6.58 & .001 \\
Partner hoarding symptoms & $0.004(0.004)$ & 1.17 & .245 \\
Participant status*actor hoarding symptoms & $-0.007(0.003)$ & -2.08 & .041 \\
Participant status*partner hoarding symptoms & $0.006(0.003)$ & 1.81 & .073
\end{tabular}

Notes. Pseudo $R^{2}=.488$. Participant status = Status of the participant (hoarding participant versus close significant other). Actor hoarding symptoms $=$ Actor overall hoarding symptom severity, as assessed with the Saving Inventory-Revised (Frost et al., 2008) total score.

Partner hoarding symptoms $=$ Partner overall hoarding symptoms, as assessed with the Saving Inventory-Revised (Frost et al., 2008) total score. 
Table 21

Two-Intercept Model Estimating Effects of Overall Hoarding Symptom Severity on Activities of Daily Living for Hoarding Participants and Close Significant Others $(n=51)$

\begin{tabular}{lccc}
\hline & $b(S E)$ & $t$ & $p$ \\
\hline CSO & $0.406(0.305)$ & 1.33 & .190 \\
Hoarding participant & $0.248(0.261)$ & 0.95 & .347 \\
CSO actor hoarding symptoms & $0.032(0.006)$ & 5.79 & 3.82 \\
Hoarding participant actor hoarding symptoms & $0.018(0.005)$ & -0.33 & .001 \\
CSO partner hoarding symptoms & $-0.002(0.005)$ & 2.24 & .030
\end{tabular}

Notes. Pseudo $R^{2}=.467$ for close significant others and .447 for hoarding participants. CSO = Close significant other. Actor hoarding symptoms $=$ Actor overall hoarding symptom severity, as assessed with the Saving Inventory-Revised (Frost et al., 2008) total score. Partner hoarding symptoms = Partner overall hoarding symptoms, as assessed with the Saving Inventory-Revised (Frost et al., 2008) total score. 
participants, meaning that there was a positive association between CSO-rated total hoarding symptom severity and hoarding participant-rated impairment in activities of daily living.

Examining total family accommodation as a mediator between total hoarding symptom severity and activities of daily living. Tests of mediation were investigated with participant status as a distinguishing variable. Participant status was entered as a fixed effect in each step.

Results of the APIMeM are displayed in Figure 5. In the first step of mediation, which tests the associations between actor and partner total hoarding symptom severity and actor total family accommodation (path a), there was a significant actor effect for hoarding participants. Hoarding participant-rated total hoarding symptom severity was positively associated with hoarding participant-rated total family accommodation. There was no significant actor effect for CSOs, and no significant partner effects for hoarding participants or CSOs. In the second step of mediation, which tests the associations between actor and partner total family accommodation and actor activities of daily living (path b), there was a significant actor effect for hoarding participants. Hoarding participant-rated total family accommodation was positively associated with hoarding participant-rated impairment in activities of daily living. There was no significant actor effect for CSOs, or partner effects for hoarding participants or CSOs. In the third step of mediation, which tests the associations between actor and partner total hoarding symptom severity and actor impairment in activities of daily living (path c), is analogous with the twointercept model that was presented in Table 21. As previously mentioned, there were significant actor effects for hoarding participants and CSOs, such that total hoarding symptom severity was positively associated with impairment in activities in daily living for both hoarding participants and CSOs. There was a significant partner effect for hoarding participants, such that CSO-rated total hoarding symptom severity was positively associated with hoarding participant-rated 


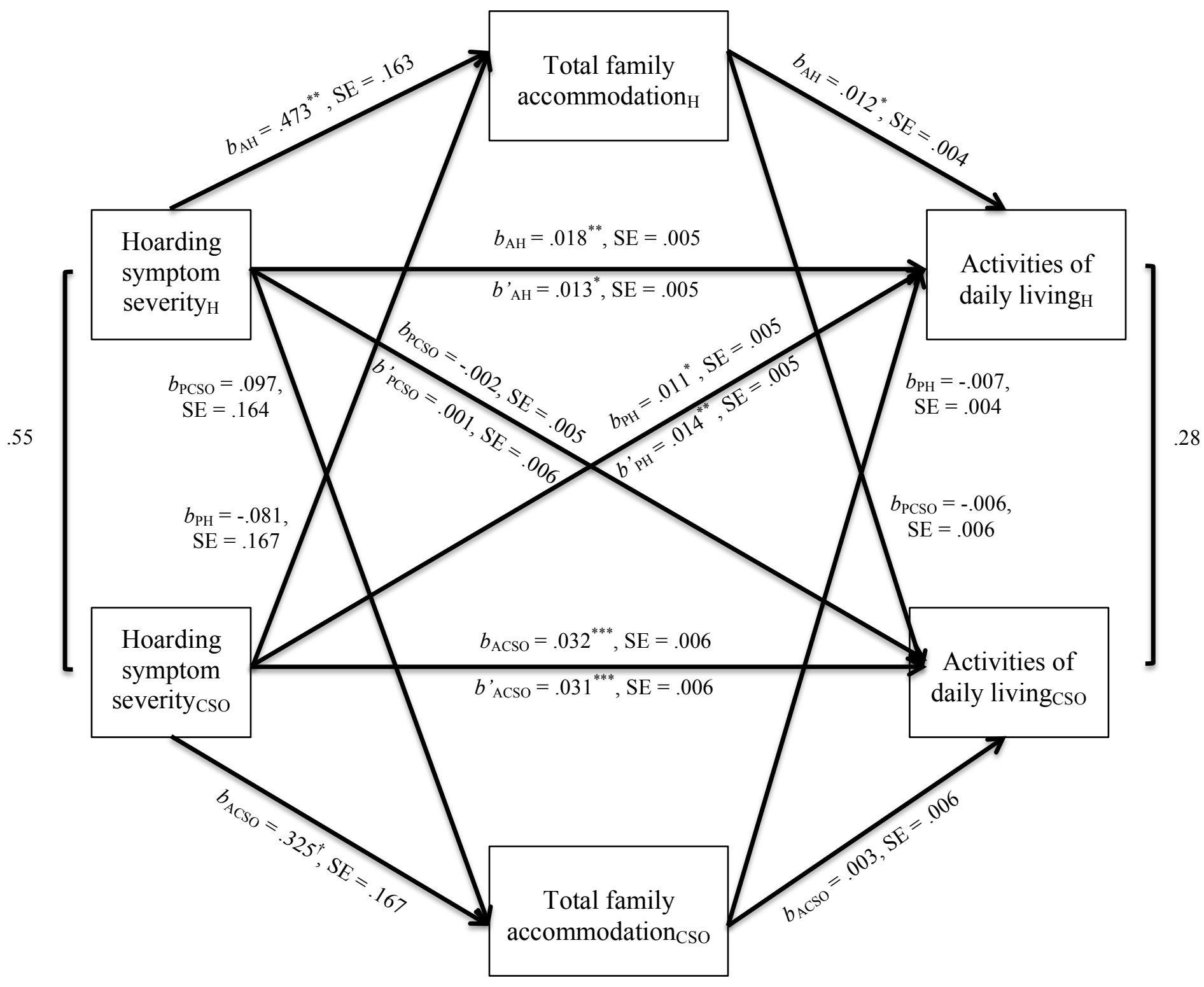

Figure 5. Actor-partner interdependence mediation model (APIMeM) assessing total family accommodation as a mediator of the association between overall hoarding symptom severity and activities of daily living for hoarding participants and close significant others. ${ }_{A}=$ Actor effect. $P=$ Partner effect. ${ }_{\mathrm{H}}=$ Hoarding participant. CSO $=$ Close significant other. ${ }^{\dagger} p=.058$. $* p<.05 . * * p<.01 . * * * p<.001$. 
impairment in activities in daily living. The partner effect for CSOs was not significant. In the final step of mediation, which tests the associations between actor and partner total hoarding symptom severity and actor activities of daily living after accounting for total family accommodation (path c'), the actor effects between total hoarding symptom severity and impairment in activities of daily living remained significant for both hoarding participants and CSOs, after accounting for total family accommodation. The partner effect for hoarding participants remained significant and the partner effect for CSOs remained nonsignificant after accounting for total family accommodation. Results of the Sobel test indicated that actor total family accommodation partially mediated the actor effect between total hoarding symptom severity and impairment in activities of daily living for hoarding participants (Table 22). The positive association between hoarding participant-rated total hoarding symptom severity and hoarding participant-rated impairment in activities of daily living was significantly decreased by hoarding participant-rated total family accommodation. Approximately $28 \%$ of the actor effect was partially mediated by actor total family accommodation for individuals with hoarding problems. Total family accommodation did not significantly mediate any of the other associations between total hoarding symptom severity and impairment in activities of daily living. ${ }^{2}$

\footnotetext{
${ }^{2}$ Similar results were found when the frequency subscale of the FAI-H was entered as a mediator of the association between total hoarding symptom severity and activities of daily living. Specifically, the frequency subscale of the FAI-H partially mediated the actor effect between total hoarding symptom severity and impairment in activities of daily living for hoarding participants. The distress/interference subscale of the FAI-H, however, did not significantly mediate the actor effect between total hoarding symptom severity and impairment in activities of daily living for hoarding participants.
} 
Table 22

Sobel Test Assessing Total Family Accommodation as a Mediator of the Association between Overall Hoarding Symptom Severity and Activities of Daily Living for Hoarding Participants and Close Significant Others $(n=51)$

\begin{tabular}{lcccc}
\hline & \multicolumn{1}{c}{ Indirect effects } & $\mathrm{SE}$ & $\mathrm{Z}$ & $p$ \\
\hline Actor-actor: CSO & $\mathrm{H}_{1} \rightarrow \mathrm{A}_{1} \rightarrow \mathrm{ADL}_{1}$ & 0.002 & 0.48 & .628 \\
$\begin{array}{l}\text { Actor-actor: hoarding } \\
\text { participant }\end{array}$ & $\mathrm{H}_{2} \rightarrow \mathrm{A}_{2} \rightarrow \mathrm{ADL}_{2}$ & 0.003 & 2.09 & .037 \\
$\begin{array}{l}\text { Partner-partner: CSO } \\
\text { Partner-partner: }\end{array}$ & $\mathrm{H}_{1} \rightarrow \mathrm{A}_{2} \rightarrow \mathrm{ADL}_{1}$ & 0.001 & 0.44 & .663 \\
$\begin{array}{l}\text { hoarding participant } \\
\text { Actor-partner: CSO }\end{array}$ & $\mathrm{H}_{2} \rightarrow \mathrm{A}_{1} \rightarrow \mathrm{ADL}_{2}$ & 0.001 & -0.56 & .575 \\
$\begin{array}{l}\text { Actor-partner: hoarding } \\
\text { participant }\end{array}$ & $\mathrm{H}_{1} \rightarrow \mathrm{A}_{1} \rightarrow \mathrm{ADL}_{1}$ & 0.003 & -0.95 & .344 \\
$\begin{array}{l}\text { Partner-actor: CSO } \\
\begin{array}{l}\text { Partner-actor: hoarding } \\
\text { participant }\end{array}\end{array}$ & $\mathrm{H}_{2} \rightarrow \mathrm{A}_{1} \rightarrow \mathrm{ADL}_{1}$ & 0.002 & -1.30 & .193 \\
\hline
\end{tabular}

Notes. $\mathrm{CSO}=$ Close significant other. $\mathrm{H}$ (predictor variable) = Overall hoarding symptom severity, as assessed with the Saving Inventory-Revised (Frost et al., 2008) total score. A (mediator) $=$ Total family accommodation, as assessed with the Family Accommodation Interview for Hoarding total score. ADL (outcome variable) $=$ Activities of Daily Living, as assessed with the Activities of Daily Living in Hoarding Scale (Frost et al., in press). ${ }_{1}=$ Dyad member $1.2=$ Dyad member 2 . 


\section{Discussion}

The purpose of the present study was to investigate the construct of family accommodation, or the processes by which family members participate in an individual's symptoms or modify personal or family routines in response to an individual's symptoms Calvocoressi et al., 1995), in hoarding, using a sample of individuals with self-reported hoarding problems and their CSOs. There were four aims for the present study. The first aim was to adapt the FAS for OCD (Calvocoressi, Mazure, Stanislav, et al., 1999; Calvocoressi, Mazure, Van Noppen, et al., 1999) for hoarding and to assess its reliability and validity (i.e., internal consistency, interrater reliability, convergent and discriminant validity). The second aim was to explore the nature (i.e., frequency and severity) of family accommodation in a hoarding sample, and assess CSOs' motivations for, and attitudes about, engaging in accommodating behaviours. The third aim was to examine the association between CSOs' accommodating behaviors and hoarding participant- and CSO-rated hoarding symptom severity, relationship satisfaction, hoarding participant- and CSO-rated functional impairment, CSOs' attribution of hoarding participants' control over hoarding behaviours, CSOs' rejecting attitudes toward the hoarding participant, and hoarding participants' and CSOs' wellbeing. The fourth aim was to investigate

the extent to which family accommodation mediated the association between hoarding symptom severity and relationship satisfaction, as well as the association between hoarding symptom severity and functional impairment.

\section{Psychometric Properties of the Family Accommodation Interview for Hoarding (FAI-H)}

Results of the present study suggest that the FAI-H is a valid and reliable measure of family accommodation in hoarding. The present study investigated the internal consistency of the FAI-H, as well as its interrater reliability; test-retest reliability (i.e., the extent to which a 
scale is stable over time; Myers \& Winter, 2002) and parallel-forms reliability (i.e., the extent to which there is agreement between two forms of a scale; Myers \& Winter, 2002) were not evaluated because there was only one form of the FAI-H and the study was cross-sectional, not longitudinal. The internal consistency for the total scale was found to be excellent, and the internal consistency of the subscales was found to be acceptable (i.e., the internal consistency for the frequency subscale was found to be acceptable and the internal consistency for the distress/interference subscale was found to be good; George \& Mallery, 2003), which suggests that the items of the FAI-H consistently measure the same latent construct. There was strong agreement between the two raters for each item of the FAI-H, as well as the total and subscale scores, providing support for the measure's interrater reliability.

The present study investigated the concurrent validity (i.e., a scale's association with an event that is measured simultaneously; Myers \& Winter, 2002) and construct validity of the FAIH; predictive validity (i.e., the scale's association with a future event; Myers \& Winter, 2002) was not assessed because the study was cross-sectional, not longitudinal. Two types of concurrent validity were assessed in the present study: convergent validity, which is defined as the extent that a scale is associated with a scale that assesses a theoretically relevant construct, and discriminant validity, which is defined as the extent that a scale is poorly associated with a scale that assesses a theoretically distinct construct (Moretz \& McKay, 2008). Results of the present study found that the FAI-H demonstrated strong convergent, as well as discriminant validity. The total and subscale scores of the FAI-H were strongly associated with the scores on a self-report measure of family accommodation in hoarding (i.e., FRHS) and significantly less associated with scores on self-report measures of hoarding participants' general stress (i.e., the stress subscale of the DASS-21) and anxiety sensitivity (i.e., ASI). Thus, given that the FAI-H 
demonstrated strong convergent, as well as discriminant validity in the present study, it is also considered to have good construct validity, which is defined as the extent to which a scale measures a particular theoretical construct (e.g., family accommodation; Myers \& Winter, 2002).

Content validity refers to the extent to which a scale's items are relevant to and represent the construct that is being measured, and is considered an important part of construct validity (Haynes et al., 1995). The importance of content validity for the validation of psychological assessment measures varies depending on how well the construct of interest has been defined and the degree to which experts within a field agree on the domains of the construct that is being measured (Haynes et al., 1995). In OCD, family accommodation has been defined as the process by which family members participate in an individual's symptoms (e.g., providing reassurance, participating in rituals, and facilitating avoidance) or modify personal or family routines in response to an individual's symptoms (e.g., taking on an individual's responsibilities, modifying cooking or cleaning practices, and helping with tasks of daily living, such as dressing and bathing; Calvoressi et al., 1995; Calvocoressi, Mazure, Stanislav et al., 1999). Measures such as the FAS for OCD have been developed to assess "classic" accommodating behaviours that are consistent with this definition. Although the conceptualization of family accommodation, which has been used in OCD, likely applies to CSOs of individuals with hoarding problems, experts within the field of hoarding have suggested that it may not provide a full description of the construct within hoarding (Steketee et al., 2013). That is, while CSOs of individuals with hoarding problems likely participate in an individual's hoarding symptoms (e.g., providing reassurance about their hoarding-related concerns, and participating in or facilitating acquiring) and modify their personal or family routines in response to an individual's hoarding symptoms (e.g., avoid entering certain rooms in the home, avoid socializing in the home, and modifying 
cooking or cleaning practices), they may also try to passively decrease (e.g., secretly discard items without the individual's permission) or aggressively decrease (e.g., initiate arguments about hoarding-related behaviours) the frequency and impact of an individual's hoarding-related behaviours (Steketee et al., 2013). Thus, future research is required to determine whether there are additional domains or facets of the construct of family accommodation in hoarding (i.e., other ways in which accommodation may manifest in CSOs of individuals with hoarding problems), and until the construct of family accommodation is more precisely defined for hoarding, it is difficult to accurately assess the content validity of the FAI-H. However, given that the FAI-H was adapted from the FAS for OCD and most items on the FAI-H assess classic accommodating behaviours, the FAI-H could potentially be considered a reliable and valid measure of classic accommodating behaviours in hoarding.

\section{Frequency and Nature of Family Accommodation in Hoarding}

CSOs in the present study endorsed a range of accommodating behaviours, with $98 \%$ of the sample endorsing at least some accommodation. This finding is consistent with previous research, which also revealed a high rate of accommodation among CSOs of individuals with OCD. For example, in the two FAS for OCD development studies, about $90 \%$ of the spouses and parents of individuals with OCD reported engaging in accommodating behaviours (Calvocoressi et al., 1995; Calvocoressi, Mazure, \& Stanislav, et al., 1999). The behaviours that CSOs reported engaging in most frequently in the present study include tolerating odd behaviours or household disruption (30.77\% engaged in this behaviour daily), refraining from saying or doing things (23.08\% engaged in this behaviour daily), and participating in or facilitating the saving of personal possessions (13.46\% engaged in this behaviour daily). The behaviours that CSOs of individuals with OCD reported engaging in most frequently include providing reassurance to the 
individual with OCD, refraining from saying or doing things because of the individual's OCD symptoms, participating in the individual's compulsive behaviours, and facilitating the individual's avoidance (Albert et al., 2010; Calvocoressi et al., 1995; Calvocoressi, Mazure, \& Stanislav, et al., 1999; Peris et al., 2008), suggesting that CSOs may engage in different types of accommodating behaviours and may engage in varying degrees of accommodation, depending on their loved one's symptomatology. This suggestion is consistent with previous research, which revealed that contamination obsessions and washing rituals among individuals with OCD were predictive of higher rates of accommodation and distress among CSOs (Albert et al., 2010; Stewart et al., 2008). Future research is needed to directly compare the rate of accommodation and distress among CSOs of individuals with nonhoarding OCD and CSOs of individuals with pure hoarding symptoms.

Previous research in the area of OCD suggests that CSOs experience significant distress when they accommodate their loved one's symptoms (Albert et al., 2010; Calvocoressi et al., 1995; Peris et al., 2008); however, the distress associated with specific behaviours was not assessed in these previous studies. The present study assessed the level of distress that was associated with each accommodating behaviour, and overall, the majority of CSOs reported at least a moderate degree of distress for most of the accommodating behaviours that they engaged in, with the exception of providing reassurance (i.e., only $42.86 \%$ reported at least moderate levels of distress) and facilitating avoidance (i.e., only $28.57 \%$ reported at least moderate levels of distress). Both the total and subscale scores of the FAI-H were significantly positively skewed, as were 14 of the 22 items. Two of the 14 significantly skewed items (i.e., the frequency of facilitating avoidance and distress/interference associated with facilitating avoidance) had very large positive skews, with a high rate of negative responses. However, given that the removal of 
these two items did not improve the internal consistency of the FAI-H total or subscale scores, these two items were retained as part of the FAI-H. Although most CSOs in the present study engaged in at least some accommodation within the past month, these results suggest that, overall, accommodations were performed infrequently and were associated with minimal distress. This is consistent with previous research, which found that nine of the 12 items on the FAS for OCD were also positively skewed (Calvocoressi, Mazure, \& Stanislav, et al., 1999). However, given that the types of accommodating behaviours that were assessed in the present study were limited to the past month and the items being assessed, it is possible that CSOs may have previously engaged in some of the accommodating behaviours that were assessed or may have engaged in other types of accommodating behaviours that were not assessed.

Although the frequency and distress/interference subscales of the FAI-H were highly correlated in the present study, there were instances in which the results of one subscale were statistically significant, while the results of the other subscale were not. Furthermore, it is also possible that these distinctions could have been larger if CSOs who have hoarding problems themselves were included in the study (i.e., it is anticipated that CSOs who have hoarding problems themselves would endorse a higher frequency of accommodating behaviours, but less distress/interference associated with the accommodating behaviours when compared with CSOs who do not have hoarding problems); however, future research with a sample that includes CSOs with the full range of hoarding problems is required to test this hypothesis. Therefore, given the differences that were observed in the present study and the possible distinctions that could occur with other samples of CSOs and individuals with hoarding problems, it is recommended that both subscales be retained at this time. However, this recommendation should continue to be assessed in future research. 
In the present study, there were no significant differences in terms of the frequency with which CSOs engaged in accommodating behaviours or the distress and interference that was associated with the accommodating behaviours when intimate partners were compared with other types of dyads (e.g., parent-child, sibling). These results suggest that CSOs may engage in accommodating behaviours with the same frequency, and experience the same level of distress and interference, regardless of the type of relationship that they have with the individual with the hoarding problem. This finding is consistent with previous OCD research, which found that accommodation did not vary based on type of relationship (spouse or parent; Calvocoressi, Mazure, Stanislav, et al., 1999), and was also present among other types of dyads (e.g., siblings; Barrett, Rasmussen, \& Healy, 2001). However, results are inconsistent with a recent hoarding study that found that behavioural modifications differed based on type of relationship (i.e., the "probable spouse/sibling" group were found to modify their behaviours more than the "probable adult children" group); no differences were found in terms of adverse consequences (Steketee et al., 2013). Given that the CSO's relationship with the individual with the hoarding problem was estimated based on age comparison in the Steketee et al. (2013) study, and results differ with the present study, future research is required to better understand the extent to which accommodation may vary based on type of relationship.

There was a significant difference in terms of frequency, but not distress/interference, when CSOs who lived with the individual with the hoarding problem were compared with CSOs who did not live with the individual with the hoarding problem. These results suggest that CSOs engage in accommodating behaviours with a greater frequency when they live with the individual with the hoarding problem than when they do not live with the individual with the hoarding problem. This finding is consistent with previous research documenting greater 
behavioural changes, as well as adverse consequences for family members who live with individuals with hoarding problems versus those who do not (Steketee et al., 2013). Given that CSOs are more likely to have frequent contact with individuals who they live with versus ones that they do not live with, it is not surprising that CSOs who live with individuals with hoarding problems engage in accommodating behaviours more frequently and report greater adverse consequences (e.g., personal health/family problems, personal/home hygiene or cleanliness problems). However, despite this increase in frequency and presence of adverse consequences, results of the present study suggest that CSOs experience the same level of distress and interference associated with accommodating, regardless of whether they live with the individual with the hoarding problem. In other words, accommodating behaviours seem to be distressing or interfering, regardless of how frequently someone does them.

\section{Close Significant Others' Motivations for Engaging in Accommodating Behaviours}

The relation between family accommodation and CSOs' own hoarding and OCD symptom severity was investigated to assess the extent to which CSOs' symptoms may influence their level of accommodation. No significant association was found between CSOs' hoarding symptom severity and family accommodation, which is inconsistent with the study hypothesis and previous research. Results of a recent Internet study documented a significant positive association between family participants' hoarding symptom severity and accommodation, suggesting that modifications are more likely among CSOs who report more severe hoarding symptoms (Steketee et al., 2013). Although the CSO's relationship with the individual with the hoarding problem was not collected in the Steketee et al. (2013) study, relationships that were derived by comparative age groupings suggest that there may be differences between the present study and the Steketee et al. (2013) study with regards to the type of CSOs that were recruited. 
That is, the majority of family participants in the Steketee et al. (2013) study were more than 20 years younger than the individual with the hoarding problem, suggesting that they were likely children of individuals with hoarding problems. The majority of participants in the present study, however, were intimate partners; thus, it is possible that the nonsignificant finding in the present study may be a reflection of the type of CSOs that were recruited. Moreover, there were also differences between the two studies in terms of the severity of CSOs' hoarding symptoms. Hoarding symptom severity for family participants in Steketee et al.'s (2013) study was assessed using the HRS-SR, and scores ranged from 5 to $45(M=14.12, S D=9.59)$. In the present study, there was a restricted range of HRS-SR scores for CSOs $(M=3.67, S D=4.19)$ because dyads were excluded if CSOs scored greater than or equal to 14 (i.e., the clinical cutoff) on the HRSSR at pre-screen. This restricted range likely influenced the results of the correlational analysis that was conducted between CSOs' hoarding symptom severity and accommodation and might also account for the nonsignificant finding in the present study. Thus, future research, which includes different types of CSOs (i.e., intimate partners, children of individuals with hoarding problems, parents of individuals with hoarding problems, and friends) with a full range of hoarding symptoms, is required to determine the extent to which CSOs own hoarding symptoms influence their accommodating behaviours.

There were significant positive associations found between the total and frequency subscale scores of the FAI-H and the total, ordering, and checking subscale scores of the CSOrated OCI-R, and between the distress/interference subscale score of the FAI-H and the ordering and checking subscale scores of the CSO-rated OCI-R. This pattern of results suggests that the severity of CSOs' OCD symptomatology, especially particular OCD symptoms, may influence the frequency with which they engage in accommodating behaviours and the distress and 
interference that they experience in response to their accommodating behaviours. These results are consistent with the study hypothesis and previous research. For example, a previous study that investigated the association between family accommodation and relatives' own OCD symptom severity in a sample of individuals with OCD and their spouses or parents found a significant positive association between relatives' own OCD symptomatology and accommodation (Calvocoressi, Mazure, Stanislav, et al., 1999). Similar results were found in a pediatric sample of children with OCD and their parents, such that parents' own OCD symptomatology was significantly positively associated with accommodation (Peris et al., 2008). No studies, to this author's knowledge, have investigated the association between family accommodation and CSOs' own OCD symptom severity in a sample of individuals with hoarding problems and their CSOs. Furthermore, although previous research has documented a significant association between CSOs' total OCD symptom severity and accommodation, no studies have investigated the association between accommodation and the various subtypes of OCD in CSOs. Results of the present study found that total accommodation was significantly associated with certain OCD subtypes (i.e., ordering and checking), but not others (i.e., washing, obsessing, hoarding, and neutralising), suggesting that some subtypes of OCD are associated with the CSO's degree of accommodation more than others. It is possible that the two subtypes of OCD that were found to be significantly associated with accommodation may be more easily triggered by hoarding-related behaviours (e.g., clutter and disorganization); thus, CSOs who experience these types of OCD symptoms may engage in greater levels of accommodation in an attempt to decrease the level of distress associated with their own OCD symptoms. However, future research is required in order to investigate this hypothesis. 
The extent to which CSOs endorsed various hoarding participant-driven (e.g., reduce the hoarding participant's distress), as well as personally-driven motivations (e.g., reduce personal distress) for engaging in accommodating behaviours was also assessed as part of the second aim of the present study. Overall, CSOs endorsed both hoarding participant-driven, as well as personally-driven motivations, which is consistent with the study hypothesis and previous research. Although previous research has provided few potential explanations for why CSOs engage in their accommodating behaviours, Calvocoressi, Mazure, Stanislav, et al. (1999) provided some potential motivations that were either patient-generated or relative-generated. For example, despite the fact that the majority of spouses and parents who engaged in accommodating behaviours did not believe that their accommodations were helpful for their loved one (i.e., they did not believe that their accommodations reduced their loved one's OCD symptoms or improved their loved one's functioning), they did believe that their accommodating behaviours (1) reduced the amount of time that their loved ones spent engaging in compulsions, (2) reduced their loved ones' level of distress, and (3) reduced their loved ones' level of anger. In addition, despite the fact that the majority of spouses and parents who engaged in accommodating behaviours did not believe that their accommodating behaviours were personally helpful or satisfying, some CSOs reported that they engaged in accommodating behaviours for their own personal reasons (i.e., it was proposed that their accommodating behaviours may have been an extension of their own personal OCD symptoms; Calvocoressi, Mazure, Stanislav, et al., 1999).

Similar to previous research (Calvocoressi, Mazure, Stanislav, et al., 1999), the majority of CSOs in the present study reported engaging in their accommodating behaviours because they believed that the behaviours reduced the hoarding participant's distress. However, the majority 
of CSOs also reported engaging in their accommodating behaviours because they believed doing so made the hoarding participant's life easier and improved the hoarding participant's functioning. In addition to these hoarding participant-driven motivations, the majority of CSOs in the present study also reported engaging in accommodating behaviours for personally-driven reasons; they reported that (1) they enjoyed supporting the hoarding participant, (2) the behaviours made their own life easier, (3) the behaviours helped them to avoid arguments with the hoarding participant, and (4) the behaviours helped to reduce personal distress. Given that many of these personally-driven motivations for engaging in accommodating behaviours could interfere with an individual with hoarding problem's response to individual treatment, and many CSOs may be reluctant to reduce their accommodating behaviours because of a individual with hoarding problem's anger, it is likely that CSOs who hold these beliefs would need to be involved in the treatment process in order for individuals with hoarding problems to respond to individual treatment (Calvocoressi, Mazure, Stanislav et al., 1999).

\section{Close Significant Others' Attitudes about Accommodating Behaviours}

Overall, almost all CSOs (i.e., 90\%) believed that their accommodating behaviours were reasonable, with the majority believing that they were helpful for the individual with the hoarding problem, as well as themselves. These results are inconsistent with previous research that has revealed that the majority of CSOs of individuals with OCD did not believe that their accommodations alleviated individuals' OCD symptoms or improved their functioning, and were not personally helpful (Calvocoressi, Mazure, Stanislav, et al., 1999). The majority of CSOs in the present study indicated that they did not need to increase or decrease their level of accommodation, suggesting that they are likely content with the frequency of their behaviours, despite the fact that the behaviours may be causing some distress or interference. Taken together, 
these results suggest that CSOs believe that their accommodations are reasonable and helpful for both the individual with the hoarding problem and themselves; as a result, many CSOs may believe that the cost of giving up their accommodating behaviours may be greater than the cost (i.e., distress or interference) associated with their current behaviours. Future research is required to test this potential hypothesis. If this hypothesis is supported, then CSOs may need to receive psychoeducation about accommodation and its reinforcing properties to increase their willingness to decrease their accommodating behaviours (Calvocoressi, Mazure, Stanislav, et al., 1999). This could be done as a separate intervention for CSOs, or as part of a family-based intervention, which includes the individual with the hoarding problem, as well as his/her CSO.

\section{Association between Family Accommodation and Hoarding Symptom Severity}

Family accommodation was positively associated with hoarding symptom severity, and posthoc analyses revealed that accommodation was greater among CSOs of individuals who endorsed hoarding symptoms that met diagnostic criteria for hoarding disorder, when compared with CSOs of individuals who did not endorse hoarding symptoms that met diagnostic criteria for hoarding disorder. Taken together, these results suggest that syndrome severity is an important variable that is associated with accommodation, which is consistent with the study hypothesis and previous research. For example, family accommodation has been found to be associated with hoarding symptom severity among CSOs of adults with hoarding problems (Steketee et al., 2013), OCD symptom severity among CSOs of children and adults with OCD (see Lebowitz et al., 2012 for a review), PTSD symptom severity among CSOs of adults with PTSD (Fredman, Vorstenbosch, Wagner, Macdonald, \& Monson, 2013), and overall anxiety symptom severity among CSOs of children with various anxiety symptoms (Lebowitz et a., 2013). Taken together, these findings provide consistent support for a positive association 
between accommodation and anxiety symptom severity, regardless of a patient's age or presenting anxiety problem. However, given that much of the research that has investigated the association between accommodation and symptom severity has been correlational in nature, future research is required to determine the causal direction of this association. Although it is possible that family accommodation may be a precursor to symptom severity, such that it may reduce the extent to which patients are exposed to anxiety-provoking situations, it is also possible that family accommodation may be a consequence of an individual's symptoms, such that family members may increase their level of accommodation as an attempt to reduce the level of anxiety or distress that their loved one is experiencing (Albert et al., 2010).

Family accommodation was significantly associated with clinician- and CSO-rated hoarding symptom severity. Clinician-rated hoarding symptom severity was significantly and positively associated with total accommodation, as well as frequency of accommodation and distress and interference associated accommodating. CSO-rated hoarding symptom severity (i.e. total hoarding, difficulty discarding, and clutter) was significantly and positively associated with total accommodation, as well as frequency of accommodation and distress and interference associated with accommodating. There were fewer significant associations between hoarding participant-rated hoarding symptom severity and accommodation. Hoarding participant-rated total hoarding symptom severity was significantly and positively associated with the frequency of accommodation, and hoarding participants' perception of their difficulties with discarding was significantly and positively associated with total accommodation, as well as frequency of accommodation. These differential associations suggest that an informant's rating of an individual's hoarding symptoms may be an important predictor of a CSO's level of accommodation. However, the significant correlations that were found between clinician-rated 
hoarding symptom severity and accommodation may be due to shared method variance (i.e., the correlation may be significant because the two interview measures were rated by the same source; Furr \& Bacharach, 2008). However, these results could also indicate that clinicians and CSOs more consistently associate accommodation with increased hoarding symptom severity, whereas individuals with hoarding problems might provide more variable responses (Storch, Geffken, Merlo, Jacob, et al., 2007). This latter explanation is consistent with past research, which has found that many individuals with hoarding problems lack insight regarding the severity of their hoarding problem (Frost, Krause, \& Steketee, 1996; Steketee et al., 2001; Tolin, Fitch, et al., 2010). Thus, it is possible that the associations were different because of individuals with hoarding problems' variable levels of insight, which is a hypothesis that should be tested in future research.

Accommodation was significantly associated with CSO-rated difficulties with discarding and clutter, but was not significantly associated with acquiring, suggesting that certain types of hoarding symptoms may be more associated with accommodation than others. These results are consistent with the results of Frost et al. (2009), who found that $15 \%$ of individuals with hoarding problems do not engage in excessive acquiring; thus, the nonsignificant association found between acquiring and accommodation may be the result of the varying levels of acquiring that are present among individuals with hoarding problems. These results are also consistent with the results of the Steketee et al. (2013) study; that is, although the Steketee et al. (2013) study found significant associations between family accommodation and total hoarding symptom severity, difficulties with discarding, clutter, and acquiring, it appears that the associations between acquiring and accommodation were weaker than the associations between difficulties with discarding and accommodation, and clutter and accommodation. Taken together, these 
results suggest that there may be differential associations between accommodation and various symptoms of hoarding. While few studies have investigated the association between family accommodation and various subtypes of OCD, results of these studies have also supported differential associations between accommodation and various subtypes of OCD, such that contamination and washing significantly predicted greater accommodation, while other OCD symptom subtypes did not (Albert et al., 2010; Flessner et al., 2011; Stewart et al., 2008). Research is currently being conducted to inform our understanding of why contamination and washing symptoms are associated with increased levels of accommodation (Lebowitz et al., 2012). Future research is also needed to clarify which hoarding symptoms are most associated with accommodation and why. For example, it is possible that accommodation may be more associated with discarding and clutter when compared with acquiring because discarding and clutter may provoke more accommodation from CSOs than acquiring. This could be because discarding and clutter are more likely to occur within the home, where the CSO presumably has more opportunity to accommodate, whereas acquiring is more likely to occur outside of the home, where the CSO presumably has less opportunity to accommodate. Additionally, it is also possible that acquiring may be more likely to be done alone, which would also result in fewer opportunities to accommodate. Future research is required to test these hypotheses.

\section{Association between Family Accommodation and Relationship Satisfaction}

Results of the present study suggest that family accommodation is associated with decreased relationship satisfaction among individuals with hoarding problems and CSOs. Specifically, increased levels of accommodation were significantly associated with greater levels of conflict for the CSO and the individual with the hoarding problem, and a decreased perception of availability of social support from the CSO for individuals with the hoarding problem. Thus, 
although CSOs reported engaging in their accommodating behaviours because they enjoy supporting the individual with the hoarding problem, these results suggest that individuals with the hoarding problem may actually feel less supported when their CSO engages in greater levels of accommodation. Moreover, these results indicate that accommodation is associated with conflict for both members of the dyad, despite the fact that the majority of CSOs reported that they engaged in their accommodating behaviours to reduce the hoarding participant's distress, as well as their own distress. In addition, distress and interference associated with accommodating were found to be significantly and negatively associated with the extent to which CSOs perceived their specific relationship as being positive, important and secure. Overall, these results are consistent with the study hypothesis and previous research that has shown a negative association between family accommodation and global family functioning (Calvocoressi, Mazure, Stanislav, et al., 1999). Significant results have also been documented between specific aspects of family functioning and accommodation. For example, family conflict has been found to be positively associated with distress associated with accommodating behaviours and negatively associated with consequences of refraining from accommodating (Peris et al., 2008). Family cohesion was negatively associated with distress associated with accommodating behaviours and family organization was negatively associated with modification of one's personal or family routine and distress associated with accommodating behaviours (Peris et al., 2008). Taken together, these results suggest that accommodation may play an important role in the positive association that has been documented between hoarding symptom severity and relationship problems (e.g., Steketee \& Frost, 2003), and may be an important treatment target to simultaneously improve hoarding symptom severity and relationship functioning. 
CSOs in the present study endorsed a high rate of rejecting attitudes toward individuals with hoarding problems, which is consistent with previous hoarding research (Tolin, Frost, Steketee, \& Fitch, 2008). Although CSOs' scores on the PRS were less than that of family members of individuals with hoarding in Tolin, Frost, Steketee, and Fitch's (2008) study, they were similar to those of CSOs of individuals with OCD (Amir et al., 2000). There were significant positive associations between family accommodation and CSOs' rejecting attitudes toward hoarding participants, which is consistent with previous research that found a significant association between family accommodation and spouses' and parents' rejecting attitudes toward individuals with OCD (Calvocoressi, Mazure, Stanislav, et al., 1999). Given that family accommodation has been found to be associated with increased negative attitudes toward individuals with hoarding problems, and these negative attitudes are likely to have negative effects on individuals with hoarding problems, as well as a dyad's relationship, it may be beneficial to provide psychoeducation to CSOs of individuals with hoarding problems about the potential negative effects of accommodation, as well as rejecting attitudes.

There were no significant associations found between family accommodation and CSOs' attributions about the hoarding participants' control over their hoarding symptoms. Although this result is inconsistent with the study hypothesis and previous research (Van Noppen \& Steketee, 2009), this inconsistent finding is likely the result of the attribution measure that was used in the present study, as it was found to have questionable internal reliability. Specifically, given that previous research found that relatives' attribution of their loved one's control over their OCD symptoms was a significant determinant of CSOs' level of accommodation (Van Noppen \& Steketee, 2009), it is possible that a similar association could be found in a sample of individuals with hoarding problems and their CSOs if an alternative measure of attribution was used. Thus, 
future research employing a more valid measure of CSOs' attribution of a loved one's symptoms (e.g., Level of Expressed Emotion Scale; Cole \& Kazarian, 1988) is needed to further elucidate the association between accommodation and CSOs' attributions of hoarding participants' control over their hoarding symptoms.

\section{Association between Family Accommodation and Functional Impairment}

Results of the present study suggest that family accommodation may be associated with certain domains of functioning, including relationship satisfaction and activities of daily living. Specifically, CSOs' report of the hoarding participant's relationship satisfaction was found to be significantly and positively associated with all aspects of accommodation, and their report of the hoarding participant's overall functioning and ability to complete activities of daily living was significantly and positively associated with the amount of distress and interference associated with accommodation. Hoarding participants' report of their relationship satisfaction was found to be significantly and positively associated with the amount of distress and interference that was associated with accommodation for the CSOs. No significant associations were found between accommodation and other domains of functioning, including lifestyle or activity involvement. These results are consistent with previous research, which documented significant associations between parents' ratings of children's functional impairment and accommodation, but not children's ratings of functional impairment and accommodation in a sample of children with OCD and their parents (Storch, Geffken, Merlo, Jacob, et al., 2007). One interpretation of these findings, which Storch, Geffken, Merlo, Jacob, et al. (2007) offered, is that symptomatic individuals might perceive themselves as being less functionally impaired because of their CSOs' increased levels of accommodation (i.e., individuals perceptions of their functional impairment may decrease as their CSOs' level of accommodation increases because the CSO is engaging in 
behaviours that may reduce the individual's distress and thus, they may not notice any decreases in terms of their functioning). Extending this interpretation, results of the present study suggest that individuals' perceptions of functioning may also vary depending on the extent to which their CSOs experience distress or interference with regard to their accommodating behaviours. This conclusion is supported by the significantly negative association that was observed between hoarding participants' relationship satisfaction and CSOs' level of distress or interference associated with accommodation. For example, it is possible that individuals with hoarding problems may only become aware of their relationship problems when their CSOs experience increased distress or interference, which individuals with hoarding problems presumably notice. Furthermore, since individuals with hoarding problems often lack insight regarding the severity of their illness (Frost et al., 1996; Steketee et al., 2001; Tolin, Fitch, et al., 2010), it is possible that they may need to observe a distressed loved one in order to appreciate the potential relationship problems associated with their hoarding problems. Thus, given the potential discrepancies that exist between impairment ratings, it is recommended that ratings from multiple sources are obtained in order to fully understand an individual's hoarding symptom severity and associated impairment (Piacentini, Bergman, Keller, \& McCracken, 2003).

\section{Association between Family Accommodation and Wellbeing}

A significant positive association between the frequency of accommodation and hoarding participant-rated anger was found in the present study; total accommodation and distress and interference associated with accommodation were not significantly associated with hoarding participant-rated anger. These results provide further support that CSOs may engage in increased levels of accommodation as an attempt to prevent arguments and reduce an individual with hoarding problem's level of distress/anger (i.e., CSOs may engage in their accommodating 
behaviours because individuals become distressed and angry when they withhold these behaviours; Storch, Geffken, Merlo, Jacob, et al., 2007); posthoc analyses that examined the association between the frequency of family accommodation and individual motivation items supported this hypothesis. However, given that the present study was cross-sectional in nature, it is also possible that CSOs' increased levels of accommodation may actually lead to increased anger among individuals with hoarding problems, which in turn could potentially lead to greater conflict and decreases in perceived support for individuals with hoarding problems. Future longitudinal research is required to elucidate the temporal direction of this association between the frequency of accommodation and hoarding participant-rated anger.

No significant associations were found between accommodation and hoarding participantrated depression, anxiety, stress, or hostility, which is inconsistent with the study hypothesis and previous research. For example, previous research has documented a significant association between children's internalizing and externalizing symptoms and accommodation in parents (Caporino et al., 2012; Storch, Geffken, Merlo, Jacob, et al., 2007), children's oppositional behaviours and accommodation in parents (Flessner et al., 2011), and individuals' depression and accommodation in family members of adults with OCD (Albert et al., 2010). There were also no significant associations found between family accommodation and personal wellbeing (i.e., depressive symptoms, anxiety symptoms, stress, or anger) for CSOs in the present study, which is inconsistent with the study hypothesis and prior research. For example, previous research has documented a significant association between various aspects of accommodation and CSO-rated depression, anxiety, hostility, and caregiver's emotional burden among CSOs of children and adults with OCD (Amir et al., 2000; Caporino et al., 2012; Flessner et al., 2011; Peris et al., 2008; Ramos-Cerqueira et a., 2008). Given that the present study assessed hoarding 
participant- and CSO-rated wellbeing with different self-report measures than these previous studies, the reliability for the anger subscale of the AQ was found to be questionable in the present study, and the sample consisted of individuals with hoarding problems and their CSOs (versus individuals with OCD and their CSOs), it is possible that the discrepancies that were found between this study and previous studies are the result of these differences in methodology. However, it is also possible that the nonsignificant associations that were found between accommodation and CSOs' wellbeing in the present study were the result of CSOs low scores on the self-report measures of wellbeing; that is, although CSOs reported a range of depression, anxiety, stress, anger, and hostility in the present study, their depression, anxiety, and stress scores were within the normal range, on average, and their anger and hostility scores were within the average range, on average. Although individuals with hoarding problems reported moderate levels of stress and depression, as well as above average levels of hostility, it is possible that the nonsignificant associations found between accommodation and hoarding participants' wellbeing may be reflective of poor insight, which has been found to be characteristic of individuals with hoarding problems, when compared with individuals with OCD (e.g., Frost et al., 1996; Storch, Lack, et al., 2007). Thus, given the discrepant methodologies, low scores on measures of wellbeing for the CSOs, and potential insight problems among individuals with hoarding, future research is needed to elucidate the association between family accommodation and personal wellbeing in individuals with hoarding problems and their CSOs. 


\section{Family Accommodation as a Potential Mediator of the Association between Hoarding}

Symptom Severity and Relationship Satisfaction, as well as Impairment in Activities of

\section{Daily Living}

Results of the present study suggest that family accommodation is one potential mechanism through which hoarding symptom severity influences relationship conflict, as well as hoarding participants' activities of daily living. Specifically, results of the present study found that family accommodation partially mediated the actor effect between hoarding symptom severity and relationship conflict, averaging across hoarding participants and CSOs. These results suggest that one's personal perception of a hoarding participant's hoarding symptom severity influences the extent to which he/she perceives that the hoarding participant's CSO engages in accommodating behaviours, which in turn, may influence the amount of conflict that exists within that dyad's relationship. In addition, results showed that family accommodation also partially mediated the actor effect between hoarding symptom severity and activities of daily living for hoarding participants, but not CSOs. These results suggest that the perception of their own hoarding symptom severity may influence the extent to which they perceive that their CSO accommodates their hoarding symptoms, which in turn, may influence the extent to which they perceive that they are able to perform activities of daily living.

This pattern of results is consistent with the study hypothesis, as well as previous research, which has found that family accommodation mediates the relationship between OCD symptom severity and parent-rated functional impairment in a sample of children with OCD and their parents (Storch, Geffken, Merlo, Jacob, et al., 2007; Caporino et al., 2012). One explanation for these findings, which was first offered by Steketee and Van Noppen (2003) and supported by Caporino and colleagues (2012), is that family accommodation may mediate the association 
between an individual's symptom severity and functional impairment because it may reduce the individual's self-efficacy, and limit their development of adaptive coping skills.

Family accommodation was not, however, investigated as a potential mediator of the association between hoarding symptom severity and relationship support and relationship depth because there was no significant association found between hoarding symptom severity and relationship support or relationship depth in the present study. Furthermore, given that neither relationship support nor relationship depth were significantly associated with total family accommodation, averaging across hoarding participants and CSOs, results suggest that accommodation may influence various aspects of relationships in different ways. However, future research is needed in order to understand the specific mediating role that accommodation plays in the association between hoarding symptom severity and relationship functioning. In addition, there was also a lack of significant partner effects in the dyadic data analyses (i.e., only two partner effects were found to be significant). There was a positively related partner effect between total family accommodation and relationship conflict, averaging across individuals with hoarding problems and CSOs, and a positively related partner effect between total hoarding symptom severity and activities of daily living for individuals with hoarding problems, but not CSOs. The lack of significant partner effects suggests that although participants' perceptions were often found to influence their own outcome, they were less likely to influence the outcome of the other member of the dyad. However, it is possible that there may be some third variable (e.g., insight) that moderates these partner effects. For example, if an individual with a hoarding problem has low insight about the severity of their problem, the probability that the individual will endorse relationship difficulties may be lower than that of individuals with greater insight about the severity of their problem. Future research is required to investigate whether there are 
any moderating variables that are contributing to the general lack of partner effects that were found in the present study.

\section{Implications of the Study Findings}

Overall, results of the present study help to increase our understanding of the complex interaction that exists between individuals with hoarding problems and their CSOs, and provide support for the cognitive-behavioural model of hoarding (Frost \& Hartl, 1996; Frost \& Steketee, 1998; Frost et al., 2003; Steketee \& Frost, 2007). Negative reinforcement is one way in which the cognitive-behavioural model of hoarding postulates that hoarding symptoms are maintained (Steketee \& Frost, 2007). For example, although acquiring may be associated with positive emotions (e.g., pleasure and excitement) and maintained through positive reinforcement, it may also prevent feelings of anxiety and thus, be maintained through negative reinforcement (e.g., if an individual does not acquire an item, he or she may experience increased feelings of anxiety due to feeling as though an opportunity has been missed; Gilliam \& Tolin, 2010). In addition, saving could be conceptualized as a behavioural avoidance strategy that is maintained through negative reinforcement, such that it may prevent unpleasant emotions (e.g., fear or anxiety) that are associated with discarding (e.g., saving may help individuals avoid negative consequences, such as a wrong decision or mistake, which they fear will occur if they were to discard specific items; Frost \& Hartl, 1996). In the present study, family accommodation was found to be positively associated with the severity of clinician- and CSO-rated overall hoarding symptoms, as well as CSOs' perceptions of an individual's difficulty with discarding and clutter, which suggests that family accommodation may also play an important maintaining role in an individual's hoarding symptoms. For example, it is possible that CSOs' accommodating behaviours (e.g., their toleration or facilitation of an individual's hoarding symptoms) may 
promote additional behavioural avoidance, which in turn maintains an individual's hoarding symptoms by preventing the unpleasant emotions that are associated with hoarding.

Moreover, results of the present study also inform our understanding of the relationship between individuals' hoarding symptoms and interpersonal functioning. The interpersonal model of OCD proposes a potential explanation for the interpersonal influences that have been found to play a role in OCD. Specifically, this model proposes an interdependent relationship between OCD symptoms, family accommodation, and CSOs' criticism/hostility (Renshaw et al., 2010). Results of the present study, which are mostly consistent with previous research, provide support for the presence of similar interpersonal influences in hoarding. For example, posthoc analyses from the present study revealed significant positive associations between hoarding symptom severity and relationship conflict for both individuals with hoarding problems and CSOs. This is consistent with previous research, which found that individuals with hoarding problems had more severe interpersonal problems when compared with healthy control participants; there were no significant differences, however, between type and severity of interpersonal problems when individuals with hoarding problems were compared with individuals with other anxiety or depressive disorders (Grisham, Steketee, \& Frost, 2008).

Research examining the association between hoarding symptom severity and CSOs' rejecting attitudes toward individuals with hoarding problems has provided further support for the potential interpersonal influences in, or affect of, hoarding. For example, CSOs who participated in Tolin, Frost, Steketee, and Fitch's (2008) study reported a high degree of rejecting attitudes toward their loved ones with hoarding problems, which was at a level that was comparable with CSOs of outpatients with schizophrenia, and hoarding symptom severity was also found to be a significant predictor of CSOs' rejecting attitudes. Posthoc analyses in the 
present study also revealed a significant positive association between CSOs' rejecting attitudes toward the individual with the hoarding problem and CSO-rated overall hoarding symptom severity; however, there were no significant associations between CSOs' rejecting attitudes and hoarding participant-rated overall hoarding symptom severity, which suggests that the CSOs' perception of the individuals hoarding symptoms may be more important than hoarding participant-rated hoarding symptom severity in terms of predicting CSOs' level of rejecting attitudes toward the individual with the hoarding problem.

Further support for a potential interpersonal influence in hoarding was documented by Steketee et al. (2013), who first identified a significant association between hoarding symptom severity and family accommodation. Results of the present study extend these findings by examining the association between family accommodation, hoarding symptom severity, relationship satisfaction, and CSOs' rejecting attitudes from the perspective of individuals with hoarding problems, as well as their CSOs. Consistent with the study hypothesis and Steketee et al.'s (2013) findings, family accommodation was found to be significantly associated with measures of hoarding symptom severity; however, as previously mentioned, CSO-rated hoarding symptom severity was more consistently associated with hoarding symptom severity than was hoarding participant-rated hoarding symptom severity. In addition, there were also significant associations between family accommodation and CSOs' rejecting attitudes toward the individual with the hoarding problem, as well as relationship satisfaction. Specifically, family accommodation was significantly and positively associated with relationship conflict for individuals with hoarding problems and CSOs, and significantly and negatively associated with relationship support for individuals with hoarding problems. Moreover, family accommodation significantly mediated the association between total hoarding symptom severity and relationship 
conflict, averaging across hoarding participants and CSOs in the present study, highlighting the important role that family accommodation may play in the association between hoarding symptom severity and relationship satisfaction. Given that the results of the present study found differential associations between CSO- and hoarding participant-rated family accommodation, hoarding symptom severity, relationship satisfaction, and CSOs' rejecting attitudes toward individuals with hoarding problems, and many individuals with hoarding problems tend to lack insight into the severity of their problem (e.g., Frost et al., 1996; Steketee et al., 2001; Tolin, Fitch, et al., 2010), it is likely that collateral reports may be particularly important when assessing interpersonal functioning with this population (Grisham et al., 2008).

Taken together, these results suggest that, like OCD, there are significant associations between hoarding symptom severity, family accommodation, and CSOs' criticism/hostility, which may be consistent with the interpersonal model that has been proposed for OCD (Renshaw et al., 2010). Specifically, results of the present study, as well as previous research, provide support for an association between hoarding symptom severity and accommodation, hoarding symptom severity and CSOs' criticism/hostility, and accommodation and CSOs' criticism/hostility. However, results of the present study, as well as previous research have been cross-sectional in nature, and have not specifically investigated the bidirectional nature of the associations that have been proposed as part of the interpersonal model of OCD. Thus, future research focusing on these interdependent associations is required to elucidate our understanding of the interpersonal factors involved in hoarding and determine whether the interpersonal model of OCD can be applied to hoarding. 


\section{Treatment Implications}

Previous research has investigated the association between family variables, including family accommodation and hostility, and treatment outcome in adults and children with OCD. Specifically, results showed that family accommodation was associated with poorer treatment outcome in both adults and children with OCD (Amir et al., 2000; Garcia et al., 2010), and treatment-refractoriness in adults with OCD (Ferrão et al., 2006). In addition, accommodating behaviours by parents and siblings of children with OCD have been found to decrease after receiving either intensive or weekly CBT (Barrett, Healy-Farrell, \& March, 2004; Storch, Geffken, Merlo, Mann, et al., 2007), and decreases in accommodation during treatment have been shown to predict treatment outcome among children with OCD (Merlo et al., 2009). Hostility among CSOs has also been found to be associated with poorer treatment outcomes and higher rates of dropout among adults with OCD and panic disorder with agoraphobia (Chambless \& Steketee, 1999); nonhostile criticism, however, was found to be associated with better treatment outcomes, which suggests that focused criticism may help to motivate individuals to complete the treatment while minimizing interpersonal stress (Renshaw, Caska, Rodrigues, \& Blais, 2012). Given that previous research has supported a significant association between family accommodation and poorer treatment outcomes, as well as hostility and poorer treatment outcomes in patients with OCD, findings of the present study may have important treatment implications.

For example, since family accommodation was found to be significantly associated with overall hoarding symptom severity and relationship satisfaction in the present study, and previous research has found that family accommodation and hostility can be responsive to treatment interventions, then it is possible that the incorporation of CSOs into current cognitive- 
behavioural treatments of hoarding may help to improve treatment outcomes. However, given that results of the present study found that CSOs have various motivations for engaging in accommodating behaviours, it is possible that, in some instances, accommodation may improve following the completion of individual treatment (Calvocoressi, Mazure, Stanislav, et al., 1999). Thus, "assessing levels of accommodation without understanding its function (e.g., to alleviate [a CSO's] own anxiety) within the family context may not provide sufficient information for optimal treatment planning" (Caporino et al., 2012, p. 141). For example, if CSOs' motivations are primarily hoarding participant-driven, then it is possible that accommodating behaviours may reduce following the completion of an individual hoarding treatment because these accommodating behaviours would be expected to decrease following an improvement in hoarding symptoms (Calvocoressi, Mazure, Stanislav, et al., 1999). However, if CSOs' motivations are personally-driven, then it is likely that CSOs will need to be incorporated as a part of treatment, as their continuation of accommodation may influence an individual with hoarding problem's treatment response (Calvocoressi, Mazure, Stanislav, et al., 1999).

A recent pilot study that investigated the efficacy of a 16-session couple-based CBT for adults with OCD and their intimate partners found that this partner-assisted intervention, which included exposure with response prevention, as well as strategies for targeting family accommodation and relationship stress, led to improvements in OCD symptoms, family accommodation, and relationship functioning (Abramowitz et al., in press). Given that similar interpersonal influences appear to play a role in the development and maintenance of hoarding, it is possible that a similar dyadic treatment, which includes individuals with hoarding problems and their CSOs, could also led to improvements in hoarding symptoms, family accommodation, as well as relationship functioning. 
However, future research is also required to determine which format of treatment delivery would be most effective for including CSOs in current cognitive-behavioural treatments for hoarding. Baucom, Shoham, Meuser, Daiuto, and Stickle (1998) reviewed three types of couple- or family-based treatments that can be used to address psychological symptoms. The first type of treatment is general couple or family therapy, which focuses specifically on improving relationship distress. The rationale for the use of general couple or family treatment is that a distressed relationship may be a stressor that maintains or exacerbates an individual's symptoms; thus, by improving the distress, clinicians may be able to indirectly improve psychological symptoms. The second type of treatment is partner- or family-assisted treatment, which uses CSOs as a surrogate therapist to help coach or assist the identified patient with out-of-session work. Although the dyad's relationship is used to help develop the identified patient's treatment plan, it is not a specific focus of partner- or family-assisted treatment. The third type of treatment is a disorder-specific couple or family treatment, which focuses on the dyad's relationship and the ways in which the dyad interacts or behaves in situations that might maintain or exacerbate symptoms of the specific psychological disorder. The dyad's relationship is a target of a disorder-specific couple or family treatment, but only when it directly influences the symptoms or the treatment. Although these three types of treatments are often described as discrete types of treatments, numerous couple and family treatments combine elements from more than one of these treatments (Baucom et al., 1998). For example, the couple-based intervention that was tested in the Abramowitz et al. (in press) study included partner-assisted exposure with response prevention (i.e., strategies from partner-assisted couple treatments), as well as strategies that could be used to target non OCD-related relationship stressors (i.e., strategies from general 
couple treatments). Future research is required to determine which type of treatment may be most appropriate and effective for individuals with hoarding problems and their CSOs.

Although previous research suggests that the incorporation of CSOs into current CBT for hoarding may be an effective strategy for improving hoarding symptoms, family accommodation and relationship functioning, it may be difficult to recruit dyads for this type of treatment given the significant difficulty that clinicians experience engaging individuals with hoarding problems in treatment (Frost \& Steketee, 1999). For example, previous research has suggested that individuals with hoarding problems may not access services due to poor insight regarding the severity of their problem and low motivation (Damecour \& Charron, 1998). While some individuals with hoarding problems may seek treatment, regardless of their level of insight, because there is distress in their relationship or CSO, other individuals may benefit from improved insight regarding the severity of their hoarding problem in order to increase their willingness to participate in treatment. Moreover, given that research has suggested that there is likely a reciprocal association between hoarding behaviours, CSOs' accommodating behaviours, and compromised relationship functioning, it is possible that modifying CSOs' behaviours could impact variables, which may also increase an individual's willingness to seek help.

Community Reinforcement and Family Training (CRAFT; Smith \& Meyers, 2004) is an empirically-supported intervention for CSOs of individuals with substance use problems, which has been found to improve treatment engagement for unmotivated individuals with substance use problems (Miller, Meyers, \& Tomgan, 1999). The intervention is based on behavioural principles and it utilizes reinforcement strategies to teach CSOs behavior change skills to modify contingencies for substance using behaviours, with the ultimate goal of the intervention being the engagement of unmotivated individuals in treatment (Smith \& Meyers, 2004). CRAFT has been 
found to be significantly more effective in engaging unmotivated individuals with substance use problems than other family-based interventions, including Al-Anon (Al-Anon Family Groups, 1984) and the Johnson Intervention Approach (Johnson, 1986); however, all three interventions were associated with reductions in CSO-rated depression, anger, and family conflict, as well as improvements in CSO-rated family cohesion and general relationship happiness (Miller et al., 1999). Since many individuals with hoarding problems experience similar problems related to poor insight into the severity of one's problem and low motivation, it is possible that CSOs of individuals with hoarding problems may also benefit from the completion of a CRAFT-based intervention. Moreover, many of the CRAFT strategies, including communication training, as well as positive reinforcement training to reinforce sober behaviours and decrease enabling behaviours, could be directly applied to CSOs of individuals with hoarding problems to decrease rejecting and hostile attitudes, as well as accommodation. Thus, by furthering our understanding of interpersonal processes, such as family accommodation, results of the present study could be used to help clinical researchers design a similar CRAFT-based intervention that could be used to increase treatment engagement in individuals with hoarding problems. Given that clinicians receive inquiries from frustrated family members more frequently than they do from individuals with hoarding problems (Tolin, Frost, Steketee, \& Fitch, 2008), this intervention may be an important first step in the treatment process for individuals with hoarding problems.

\section{Strengths and Limitations of the Present Study}

Overall, results of the present study extend current literature and further our understanding regarding the role of family accommodation in hoarding, and the complex interaction that exists between individuals with hoarding problems and their CSOs. The present study had several methodological strengths, including the dyadic nature of the study, which 
included both individuals with hoarding problems and their CSOs. Given that the two previous studies (Steketee et al., 2013; Wilbram et al., 2008) that investigated the role of family accommodation in hoarding recruited only the CSOs of individuals with hoarding problems, this study extends these previous findings by incorporating both members of the dyad to further elucidate the associations between hoarding behaviours, accommodation, and relationship functioning. Second, previous research has recommended the FAS for OCD as the gold standard assessment tool for assessing family accommodation among individuals with OCD (Pinto et al., in press). The interview (i.e., FAI-H) that was used to assess accommodation in hoarding was adapted from this gold standard instrument, and was found to be a valid and reliable measure of accommodating behaviours in this sample. Third, the statistical analysis that was used to test the mediating role of accommodation in this study (i.e., APIMeM; Ledermann et al., 2011) was developed specifically for dyadic data and takes into account the influence of each member on the other member's scores.

The present study, however, is not without its limitations. First, the majority of hoarding participants and CSOs were female and Caucasian. Second, causal statements cannot be made regarding the specific role of family accommodation in hoarding given the cross-sectional design of the study. Future longitudinal studies are required to further elucidate the causal relationship between hoarding behaviours, family accommodation, and relationship functioning. Third, multiple comparisons were needed to test the study hypotheses, which may have inflated type I error; however, given that many of the findings are consistent with previous OCD research, it is likely that many of the observed effects are genuine. Future research is required to provide further support for the associations that were observed in the present study. Fourth, given that both the individual with the hoarding problem and the CSO had to be willing to participate in 
order to be eligible to participate in the study, results cannot be generalized to all CSOs of individuals with hoarding problems. For example, at least 20 CSOs who inquired about the present study were unable to participate because the individual with the hoarding problem refused to participate. In addition, given that CSOs were excluded if they endorsed clinically significant hoarding symptoms on the pre-screen questionnaire, future research is required to assess the study hypotheses among dyads in which both members have clinically significant hoarding symptoms. For example, by including CSOs with varying degrees of hoarding symptoms, we could better assess the extent to which CSOs' own hoarding symptoms influence accommodation and relationship functioning. Finally, the sample consisted of nontreatment seeking individuals with hoarding problems and their CSOs, with a subset of the sample $(n=16)$ including hoarding participants who endorsed hoarding symptoms that did not meet diagnostic criteria for hoarding disorder, as defined by Tolin, Frost, Steketee, Gray, et al., 2008. Posthoc analyses found that CSOs of individuals who endorsed hoarding symptoms that met diagnostic criteria for hoarding disorder reported greater total accommodation and more distress and interference associated with accommodation, when compared with CSOs of individuals who did not endorse hoarding symptoms that met diagnostic criteria for hoarding disorder, providing additional support suggesting that symptom severity plays an important role in accommodation. Moreover, similar trends were found when analyses were repeated excluding the dyads who consisted of individuals who did not endorse hoarding symptoms that met diagnostic criteria for hoarding disorder and their CSOs; thus, even though a subset of the sample did not endorse hoarding symptoms that met diagnostic criteria for hoarding disorder, it is likely that similar results would be found in a sample of individuals who endorsed hoarding symptoms that met diagnostic criteria for hoarding disorder and their CSOs. However, given the reduced sample size 
$(n=36)$ and lack of sufficient statistical power, future research that includes a larger sample of individuals with hoarding symptoms that meet diagnostic criteria for hoarding disorder and their CSOs is needed to confirm that these results can be replicated using a sample that consists of individuals with hoarding disorder and their CSOs. In addition, future research that includes treatment-seeking individuals is also necessary to help us understand the influence that family accommodation and relationship factors (e.g., hostility) may have on treatment outcome and dropout in a hoarding sample.

\section{Conclusion}

The purpose of the present study was to investigate the nature of CSOs' accommodating behaviours in a sample of individuals with self-reported hoarding problems and their CSOs and explore the extent to which these behaviours were associated with hoarding symptom severity, relationship satisfaction, functional impairment, and wellbeing. Results of the present study suggest that the FAI-H is a valid and reliable measure of the construct of family accommodation in hoarding. Most CSOs reported engaging in accommodating behaviours, and those who lived with the individual with the hoarding problem were found to engage in accommodating behaviours more frequently than those who did not live with the individual with the hoarding problem. Various motivations for engaging in accommodating behaviours were endorsed, and significant associations were found between family accommodation and hoarding symptom severity, relationship conflict, CSOs' rejecting attitudes towards the individual with hoarding problems, relationship functioning, activities of daily living, and hoarding participant-rated anger. In addition, family accommodation was found to be a partial mediator of the association between hoarding symptom severity and relationship conflict, and between hoarding symptom severity and impairment in activities of daily living for individuals with hoarding problems, but not CSOs. 
Overall, results of the present study increase our understanding of the complex interaction that exists between individuals with hoarding problems and their CSOs, and provide initial support for an association between hoarding symptom severity, family accommodation, and interpersonal functioning. In addition, results have important treatment implications, such that by further understanding interpersonal processes (e.g., family accommodation) we may be able to improve current treatment outcomes or increase treatment engagement among individuals with hoarding problems. 
Appendix A

\title{
FAMILY ACCOMMODATION INTERVIEW FOR HOARDING (FAI-H)
}

\author{
Developed by: \\ Valerie Vorstenbosch, MA, Martin M. Antony, PhD, Candice M. Monson, $\mathrm{PhD}$, and Karen \\ Rowa, $\mathrm{PhD}$
}

\section{Copyright and Permissions}

The Family Accommodation Interview for Hoarding Copyright $(\mathcal{} 2012$ by Valerie Vorstenbosch, MA, Martin M. Antony, PhD, Candice M. Monson, $\mathrm{PhD}$, and Karen Rowa, $\mathrm{PhD}$

The initial items (items 1-7, 9-11) and format of the Family Accommodation Interview for Hoarding were adapted from the Family Accommodation Scale for Obsessive-Compulsive Disorder Interviewer-Rated (FAS-IR; Developed by Lisa Calvocoressi, Carolyn M. Mazure, Barbara Van Noppen, \& Lawrence Price, copyright 1999), with permission.

The Family Accommodation Interview for Hoarding also includes the Hoarding Rating ScaleInterview (Tolin, Frost, \& Steketee, 2010). 


\section{Family Accommodation Interview for Hoarding}

Introduction and General Instructions for the Family Member:

The purpose of this interview is to learn about the ways in which you may modify your behavior or routines because of [name of hoarding individual]'s characteristics. During this interview, I will ask you about the types of characteristics that (name of the hoarding individual) has displayed during the past month, and then I will ask you about the ways in which you may have responded to these behaviors. If at any time, you are uncertain about what I am asking, please let me know and I will try to clarify the question for you. Do you have any questions before we begin?

Administer the Hoarding Rating Scale-Interview (Tolin, Frost, \& Steketee, 2010) to assess the family member's perceptions of the hoarding individual's symptoms during the past month. Record specific symptoms on the sheet entitled patient symptom list. 
This measure may not be used without permission from the authors

\section{Patient Symptom List}

Describe symptoms reported by the family member during the Hoarding Rating Scale-Interview and refer to this list when posing the remaining questions.

EXCESSIVE ACQUISITION OF POSSESSIONS: Individuals with hoarding problems may acquire possessions by buying items, obtaining free items (such as free brochures, giveaways or discarded items), or stealing items. Provide examples of how (name of hoarding individual) has acquired possessions and the types of possessions that have been acquired during the past month.

DIFFICULTY DISCARDING: Individuals with hoarding problems tend to have persistent difficulty discarding or parting with personal possessions, even those of apparently useless or limited value, due to strong urges to save items, distress, and/or indecision associated with discarding. Provide examples of how (name of hoarding individual) has had difficulty discarding during the past month.

CLUTTER: Individuals with hoarding problems tend to accumulate a large number of possessions that fill up and clutter active living areas of the home, workplace, or personal surroundings (for example, the office, vehicle, or yard), which prevent normal use of the living space. Describe (name of hoarding individual)'s clutter during the past month.

OTHER HOARDING-RELATED PROBLEMS (avoidance, indecisiveness, overvalued sense of responsibility, etc.) 
This measure may not be used without permission from the authors

\section{Family Member's Report of Accommodating Behaviors}

Instructions for the Family Member: You told me that (name of hoarding individual) has the following characteristics or behaviors (review the patient symptom list). I am now going to ask you about some ways in which you may have responded to these behaviors during the past month. (Formulate examples of accommodation for each question using the specific symptoms on the patient symptom list).

\section{PROVIDE REASSURANCE}

During the past month, when (name of hoarding individual) has expressed worries, fears, or doubts related to acquiring, discarding, or clutter, have you reassured him/her that s/he doesn't have to worry, or that there are no grounds for his/her concerns (e.g., promising your relative that you will help them find an object again if they lost it)?

Please specify:

Frequency: During the past month, on how many occasions did you provide reassurance to (name of hoarding individual) that was directly related to acquiring, discarding, or clutter? [Do not include instances in which you provided more general reassurance that $\mathrm{s} / \mathrm{he}$ will overcome his/her symptoms or feel better soon, or reassurance about matters unrelated to hoarding]
$0 . \quad$ Never
1. Once or twice per month
2. Once or twice per week
3. Several times per week
4. Daily

Distress/Interference: How much did providing reassurance to the hoarding individual bother you? (How hard did you try to not provide reassurance?) How much did the reassuranceseeking interfere with your life?
0. Not at all
1. Mild; minimal distress or disruption of activities
2. Moderate; some distress or disruption of activities
3. Severe; considerable distress or disruption of activities
4. Extreme; marked distress or disruption of activities

Please explain why you provide reassurance to the hoarding individual: 


\section{WAIT FOR THE HOARDING INDIVIDUAL}

During the past month, did you wait for (name of hoarding individual) to complete hoardingrelated behaviors, resulting in interference with plans you had made (e.g., when leaving the house, or shopping)?

Please specify:

Frequency: During the past month, how many times did you wait for (name of hoarding individual) because of his/her hoarding?

0. Never

1. Once or twice per month

2. Once or twice per week

3. Several times per week

4. Daily

Distress/Interference: How much did waiting for (name of hoarding individual) bother you? (How much time did you spend waiting for the hoarding individual?) How much did waiting for (name of hoarding individual) interfere with your life?

0. Not at all

1. Mild; minimal distress or disruption of activities

2. Moderate; some distress or disruption of activities

3. Severe; considerable distress or disruption of activities

4. Extreme; marked distress or disruption of activities

Please explain why you wait for the hoarding individual:

\section{PARTICIPATE IN/FACILITATE THE HOARDING INDIVIDUAL'S ACQUIRING}

During the past month, did you deliberately engage in (name of hoarding individual)'s acquiring or in behaviors that you consider senseless because you thought s/he would want you to do these things?

Examples include participating in shopping excursions, giving (name of hoarding individual) money to acquire possessions, making extra space for items, or bringing home something that is likely to be hoarded?

Please specify: 
This measure may not be used without permission from the authors

Frequency: During the past month, how many times did you participate in or facilitate behaviors related to (name of hoarding individual)'s acquiring?
0. Never
1. Once or twice per month
2. Once or twice per week
3. Several times per week
4. Daily

Distress/Interference: How much did participating in or facilitating (name of hoarding individual)'s acquiring bother you? (How much time did you spend participating in or facilitating acquiring?) (How much money did you give to the hoarding individual?) How much did participating in or facilitating (name of hoarding individual)'s acquiring interfere in your life?
0. $\quad$ Not at all
1. Mild; minimal distress or disruption of activities
2. Moderate; some distress or disruption of activities
3. Severe; considerable distress or disruption of activities
4. Extreme; marked distress or disruption of activities

Please explain why you participate in or facilitate the hoarding individual's acquiring:

\section{PARTICIPATE IN/FACILITATE THE SAVING OF PERSONAL POSSESSIONS}

During the past month, did your actions contribute to the (name of hoarding individual)'s saving behaviors (e.g., did you do anything to make it easier for (name of hoarding individual) to avoid making decisions or organizing/categorizing his/her possessions)?

Examples include not throwing out (name of hoarding individual)'s items without their permission, or keeping items longer because of (name of hoarding individual)'s hoarding.

Please specify:

Frequency: During the past month, how many times did your actions contribute to (name of hoarding individual)'s saving behaviors?
0. Never
1. Once or twice per month
2. Once or twice per week
3. Several times per week
4. Daily 
Distress/Interference: How much did your facilitation of (name of hoarding individual)'s saving bother you? (Did you go out of your way to help the hoarding individual save items?) How much did facilitating saving interfere with your life?
$0 . \quad$ Not at all
1. Mild; minimal distress or disruption of activities
2. Moderate; some distress or disruption of activities
3. Severe; considerable distress or disruption of activities
4. Extreme; marked distress or disruption of activities

Please explain why you participate in or facilitate the hoarding individual's saving:

\section{FACILITATE AVOIDANCE}

During the past month, did you get involved in (name of hoarding individual)'s efforts to avoid people, places or things that are hoarding related?

Please specify:

Frequency: During the past month, how many times did you do something that helped (name of hoarding individual) avoid? [Do not include instances in which you participated in acquiring or did something that facilitated the hoarding individual's acquiring or saving, as noted in the previous two questions].
$0 . \quad$ Never
1. Once or twice per month
2. Once or twice per week
3. Several times per week
4. Daily

Distress/Interference: How much has your involvement in (name of hoarding individual)'s avoidance bothered you? (Have you had to rearrange or cancel plans because of the hoarding individual's avoidance?) How much as your involvement in (name of hoarding individual)'s avoidance interfered in your life?
$0 . \quad$ Not at all
1. Mild; minimal distress or disruption of activities
2. Moderate; some distress or disruption of activities
3. Severe; considerable distress or disruption of activities
4. Extreme; marked distress or disruption of activities

Please explain why you facilitate avoidance: 


\section{TOLERATE ODD BEHAVIORS/HOUSEHOLD DISRUPTION}

During the past month, did you tolerate odd behaviors on (name of hoarding individual)'s part (e.g., rummaging through dumpsters) or did you put up with conditions in your home because of (name of hoarding individual)'s hoarding behavior (e.g., tolerate clutter in the home)?

Please specify:

Frequency: During the past month, how often did you tolerate odd behaviors or unusual conditions in your home because of (name of hoarding individual)'s hoarding? [This question is specific to behaviors or conditions that you allow to occur].
0. Never
1. Once or twice per month
2. Once or twice per week
3. Several times per week
4. Daily

Distress/Interference: How much did your tolerance of odd behaviors or unusual conditions bother you? (To what extent did you tolerate odd behaviors or unusual conditions because of (name of hoarding individual)'s hoarding?) How much did your tolerance of these odd behaviors or unusual conditions interfere in your life?
0. $\quad$ Not at all
1. Mild; minimal distress or disruption of activities
2. Moderate; some distress or disruption of activities
3. Severe; considerable distress or disruption of activities
4. Extreme; marked distress or disruption of activities

Please explain why you tolerate odd behaviours:

\section{REFRAIN FROM SAYING/DOING THINGS}

During the past month, were there things that you did not do or say because of (name of hoarding individual)'s hoarding? For example, did you stop yourself from entering some or all areas of the house, refrain from physical contact with the hoarding individual's possessions, or avoid conversation topics related to the hoarding individual's hoarding behaviors?

Please specify: 
Frequency: During the past month, how often did you stop yourself from saying or doing things because of (name of hoarding individual)'s hoarding?
$0 . \quad$ Never
1. Once or twice per month
2. Once or twice per week
3. Several times per week
4. Daily

Distress/Interference: How much did refraining from doing or saying things bother you? (How hard was it for you to refrain?) How much did refraining from doing or saying things interfere with your life?
$0 . \quad$ Not at all
1. Mild; minimal distress or disruption of activities
2. Moderate; some distress or disruption of activities
3. Severe; considerable distress or disruption of activities
4. Extreme; marked distress or disruption of activities

Please explain why you refrain from saying/doing things:

\section{MAKE EXCUSES FOR THE HOARDING INDIVIDUAL'S BEHAVIOR}

During the past month, did you make excuses for (name of hoarding individual)'s behavior or try to manage his/her relationships?

Please specify:

Frequency: During the past month, how often did you make excuses for (name of hoarding individual)'s behavior?
0. $\quad$ Never
1. Once or twice per month
2. Once or twice per week
3. Several times per week
4. Daily

Distress/Interference: How much did making excuses for (name of hoarding individual)'s behavior bother you? (How hard did you try to make excuses?) How much did making excuses for (name of hoarding individual) interfere with your life? 
This measure may not be used without permission from the authors
0. Not at all
1. Mild; minimal distress or disruption of activities
2. Moderate; some distress or disruption of activities
3. Severe; considerable distress or disruption of activities
4. Extreme; marked distress or disruption of activities

Please explain why you make excuses for the hoarding individual's behaviour:

\section{HELP THE HOARDING INDIVIDUAL WITH TASKS OF DAILY LIVING OR SIMPLE DECISIONS}

During the past month, did you help (name of hoarding individual) complete simple tasks of daily living or make simple decisions when his/her ability was impaired by hoarding; for example, helping him/her clean the house, helping him/her prepare meals or bathe?

Please specify:

Frequency: During the past month, on how many occasions did you help (name of hoarding individual) with simple tasks or decisions because s/he was impaired by hoarding?
0. Never
1. Once or twice per month
2. Once or twice per week
3. Several times per week
4. Daily

Distress/Interference: How much has helping (name of hoarding individual) with simple tasks or decisions bothered you? (How much time did you spend helping the hoarding individual?) How much has helping (name of hoarding individual) with simple tasks or decisions interfered with your life?

\section{0. $\quad$ Not at all}

1. Mild; minimal distress or disruption of activities

2. Moderate; some distress or disruption of activities

3. Severe; considerable distress or disruption of activities

4. Extreme; marked distress or disruption of activities

Please explain why you help the hoarding individual with these tasks: 


\section{TAKE ON THE HOARDING INDIVIDUAL'S RESPONSIBILITIES}

During the past month, did you take on tasks that are (name of hoarding individual)'s responsibility but cannot be adequately performed because of his/her hoarding? Examples include taking out the trash, grocery shopping, taking care of his/her children, paying his/her bills. [Do not include simple tasks of daily living for the hoarding individual, as noted under the previous question].

Please specify:

Frequency: During the past month, how often did you take on (name of hoarding individual)'s responsibilities due to hoarding?
0. Never
1. Once or twice per month
2. Once or twice per week
3. Several times per week
4. Daily

Distress/Interference: How much did taking on these responsibilities bother you? (To what extent did you take on (name of hoarding individual)'s responsibilities?) How much did taking on (name of hoarding individual)'s responsibilities interfere with your life?
0. Not at all
1. Mild; minimal distress or disruption of activities
2. Moderate; some distress or disruption of activities
3. Severe; considerable distress or disruption of activities
4. Extreme; marked distress or disruption of activities

Please explain why you take on the hoarding individual's responsibilities:

\section{MODIFY YOUR PERSONAL OR FAMILY ROUTINE}

During the past month, did you modify your leisure time activities, or your work or family responsibilities because of (name of hoarding individual)'s hoarding (e.g., spending less time socializing in the home, being unable to engage in hobbies, or work in the home)? Did you modify any family routines because of (name of hoarding individual)'s hoarding (e.g., changing your family's cooking or cleaning practices)?

Please specify:

Frequency: During the past month, how often did you modify your personal or family routine because of (name of hoarding individual)'s hoarding? 
$0 . \quad$ Never

1. Once or twice per month

2. Once or twice per week

3. Several times per week

4. Daily

Distress/Interference: How much did these modifications bother you? (To what extent did you modify your personal or family routine because of (name of hoarding individual)'s hoarding?) How much did these modifications interfere with your life?

0. Not at all

1. Mild; minimal distress or disruption of activities

2. Moderate; some distress or disruption of activities

3. Severe; considerable distress or disruption of activities

4. Extreme; marked distress or disruption of activities

Please explain why you modify your personal or family routine:

\section{OTHER}

Are there any other ways in which you may have modified your behavior or routines because of (name of hoarding individual)'s characteristics in the past month?

Please specify:

Frequency: During the past month, how often did you engage in [other behavior]?
0. Never
1. Once or twice per month
2. Once or twice per week
3. Several times per week
4. Daily

Distress/Interference: How much did engaging in [other behavior] bother you? How much did engaging in [other behavior] interfere with your life?
0. $\quad$ Not at all
1. Mild; minimal distress or disruption of activities
2. Moderate; some distress or disruption of activities
3. Severe; considerable distress or disruption of activities
4. Extreme; marked distress or disruption of activities 
This measure may not be used without permission from the authors

Please explain why you engage in [other behaviour]: 


\section{Scoring Sheet}

\begin{tabular}{|c|c|c|}
\hline Item & Frequency & Distress/Interference \\
\hline 1. Provide reassurance & $\begin{array}{lllll}0 & 1 & 2 & 3 & 4\end{array}$ & $\begin{array}{lllll}0 & 1 & 2 & 3 & 4\end{array}$ \\
\hline $\begin{array}{l}\text { 2. Wait for the hoarding } \\
\text { individual }\end{array}$ & $\begin{array}{lllll}0 & 1 & 2 & 3 & 4\end{array}$ & $\begin{array}{lllll}0 & 1 & 2 & 3 & 4\end{array}$ \\
\hline $\begin{array}{l}\text { 3. Participate in or } \\
\text { facilitate acquiring }\end{array}$ & $\begin{array}{lllll}0 & 1 & 2 & 3 & 4\end{array}$ & $\begin{array}{lllll}0 & 1 & 2 & 3 & 4\end{array}$ \\
\hline $\begin{array}{l}\text { 4. Participate in or } \\
\text { facilitate saving }\end{array}$ & $\begin{array}{lllll}0 & 1 & 2 & 3 & 4\end{array}$ & $\begin{array}{lllll}0 & 1 & 2 & 3 & 4\end{array}$ \\
\hline 5. Facilitate avoidance & $\begin{array}{lllll}0 & 1 & 2 & 3 & 4\end{array}$ & $\begin{array}{lllll}0 & 1 & 2 & 3 & 4\end{array}$ \\
\hline $\begin{array}{l}\text { 6. Tolerate odd behaviors } \\
\text { or household disruption }\end{array}$ & $\begin{array}{lllll}0 & 1 & 2 & 3 & 4\end{array}$ & $\begin{array}{lllll}0 & 1 & 2 & 3 & 4\end{array}$ \\
\hline $\begin{array}{l}\text { 7. Refrain from saying or } \\
\text { doing things }\end{array}$ & $\begin{array}{lllll}0 & 1 & 2 & 3 & 4\end{array}$ & $\begin{array}{lllll}0 & 1 & 2 & 3 & 4\end{array}$ \\
\hline 8. Make excuses & $\begin{array}{lllll}0 & 1 & 2 & 3 & 4\end{array}$ & $\begin{array}{lllll}0 & 1 & 2 & 3 & 4\end{array}$ \\
\hline $\begin{array}{l}\text { 9. Help with tasks of daily } \\
\text { living or decisions }\end{array}$ & $\begin{array}{lllll}0 & 1 & 2 & 3 & 4\end{array}$ & $\begin{array}{lllll}0 & 1 & 2 & 3 & 4\end{array}$ \\
\hline 10. Take on responsibilities & $\begin{array}{lllll}0 & 1 & 2 & 3 & 4\end{array}$ & $\begin{array}{lllll}0 & 1 & 2 & 3 & 4\end{array}$ \\
\hline $\begin{array}{l}\text { 11. Modify personal or } \\
\text { family routine }\end{array}$ & $\begin{array}{lllll}0 & 1 & 2 & 3 & 4\end{array}$ & $\begin{array}{lllll}0 & 1 & 2 & 3 & 4\end{array}$ \\
\hline
\end{tabular}

Frequency subscale (sum of frequency items):

Distress/interference subscale (sum of distress/interference items):

Total score (sum of all items): 


\section{Appendix B \\ Interview Assessing Motivations for, and Attitudes About, Engaging in Accommodating Behaviours}

Review the types of accommodating behaviours that the CSO endorsed on the Family Accommodation Interview for Hoarding.

Ask the CSO to rate the extent to which he/she believes each of the following statements is true on a 5-point scale, ranging from 0 (not at all) to 4 (extremely).

\section{Hoarding individual-driven subscale}

1. You engage in these behaviours to help reduce (name of hoarding individual)'s hoarding behaviours.
0
1
2
3
4

Not at all

Moderately

Extremely

Please describe how your behaviours help to reduce (name of hoarding individual)'s hoarding behaviours.

2. You engage in these behaviours to improve (name of hoarding individual)'s functioning (e.g., at work, or completing daily responsibilities).
0
1
2
3
4

$\begin{array}{lll}\text { Not at all } & \text { Moderately } & \text { Extremely }\end{array}$

Please describe how your behaviours improve (name of hoarding individual)'s functioning. 
3. You engage in these behaviours because (name of hoarding identified partner) becomes distressed when you do not do the behaviours.
0
1
2
3
4

Not at all

Moderately

Extremely

Please describe how (name of hoarding individual) shows his/her distress.

4. You engage in these behaviours to make (name of hoarding individual)'s life easier.

0

Not at all

Please describe how your behaviours make (name of hoarding individual)'s life easier.
4

Extremely

5. You engage in these behaviours because (name of hoarding individual) spends more time engaging in hoarding-related behaviours when you do not complete these behaviours.

0

1

2

Moderately

Not at all

Please describe what happens when you do not engage in these behaviours. 
Please describe how these behaviours help to make your life easier.

7. You engage in these behaviours because you find it personally distressing when you do not do them.
0
1
2
3
4

Not at all

Moderately

Extremely

Please describe how you find these behaviours personally distressing.

8. You engage in these behaviours because you enjoy supporting your CSO.

0

1

2

Moderately
3

4

Not at all

Please describe how these behaviours support your CSO.

9. You engage in these behaviours because you want to avoid getting into arguments with your CSO.

0

Not at all
1

2

Moderately
4

Extremely

Please describe how these behaviours help you avoid arguments with your CSO. 
10. You engage in these behaviours because you enjoy doing them.

0

1

3

4

Not at all

Moderately

Extremely

Please describe how you find these behaviours satisfying.

\section{CSOs' beliefs about the utility of accommodating}

11. To what extent do you believe that your behaviours are reasonable?

0

Not at all

Please explain.
1

2

Moderately
3 4

Extremely

12. To what extent do you believe that your behaviours are helpful for your CSO?

0

Not at all
1
3

Moderately
4

Extremely

Please describe why you think your behaviours are helpful for your CSO.

13. To what extent do you believe that your behaviours are helpful for you?

0

Not at all
1

2

Moderately
3

4

Extremely 
Please describe why you think your behaviours are helpful for you.

14. To what extent do you believe that you need to decrease your behaviours?

0

1

2

Moderately
4

Extremely

Not at all

Please describe why you think you need/do not need to decrease your behaviours.

15. To what extent do you believe that you need to increase your behaviours?

0

Not at all 1 2 Moderately
4

Extremely

Please describe why you think you need/do not need to increase your behaviours. 


\section{Appendix C}

\section{Hoarding Attribution Questionnaire (HAQ)}

Please indicate how much you agree or disagree with each statement about your partner's hoarding-related behaviours:

1

Strongly

Disagree
$2 \quad 3$

Disagree
4

Agree
5

Strongly

1. My partner should be able to control his/her hoarding behaviours.

2. My partner's hoarding behaviours are due to a mental health problem. (R)

3. My partner could discard more possessions, if he/she wanted to.

4. My partner needs psychological help in order to improve his/her hoarding problem. (R)

5. My partner can control whether his/her hoarding gets better or worse.

6. My partner hoards because of laziness. 


\section{References}

Abramowitz, J. S., Baucom, D. H., Boeding, S., Wheaton, M. G., Pukay-Martin, N. D., Fabricant, L. E., .. . Fischer, M. (in press). Treating obsessive-compulsive disorder in intimate relationships: A pilot study of couple-based cognitive-behavior therapy. Behavior Therapy.

Abramowitz, J. S., \& Deacon, B. J. (2006). Psychometric properties and construct validity of the Obsessive-Compulsive Inventory-Revised: Replication and extension with a clinical sample. Journal of Anxiety Disorders, 20, 1016-1035. doi: 10.1016/j.janxdis.2006.03.001

Al-Anon Family Groups. (1984). Al-Anon faces alcoholism. New York, NY: Author.

Albert, U., Bogetto, F., Maina, G., Saracco, P., Brunatto, C., \& Mataix-Cols, D. (2010). Family accommodation in obsessive-compulsive disorder: Relation to symptom dimensions, clinical and family characteristics. Psychiatry Research, 179, 204-211. doi:

10.1016/j.psychres.2009.06.008

American Psychiatric Association. (2013). Diagnostic and statistical manual of mental disorders ( $5^{\text {th }}$ ed.). Washington, DC: Author.

Amir, N., Freshman, M., \& Foa, E. B. (2000). Family distress and involvement in relatives of obsessive-compulsive disorder patients. Journal of Anxiety Disorders, 14, 209-217. doi: 10.1016/S0887-6185(99)00032-8

Antony, M. M., Bieling, P. J., Cox, B. J., Enns, M. W., \& Swinson, R. P. (1998). Psychometric properties of the 42-item and 21-item versions of the Depression Anxiety Stress Scales in clinical groups and a community sample. Psychological Assessment, 10, 176-181. doi: $10.1037 / 1040-3590.10 .2 .176$ 
Bailer, J., Rist, F., Bräuer, W., \& Rey, E. R. (1994). Patient Rejection Scale: Correlations with symptoms, social disability and number of rehospitalizations. European Archives of Psychiatry Clinical Neuroscience, 244, 45-48. doi: 10.1007/BF02279811

Baron, R. M., \& Kenny, D. A. (1986). The moderator-mediator variable distinction in social psychological research: Conceptual, strategic, and statistical considerations. Journal of Personality and Social Psychology, 51, 1173-1182. doi: 10.1037/0022-3514.51.6.1173

Barret, P. M., Healy-Farrell, L., \& March, J. S. (2004). Cognitive-behavioral family treatment of childhood obsessive-compulsive disorder: A controlled trial. Journal of the American Academy of Child and Adolescent Psychiatry, 43, 46-62. doi:

10.1097/01.CHI.0000096367.43887.13

Barrett, P. M., Rasmussen, P. J., \& Healy, L. (2001). The effect of obsessive-compulsive disorder on sibling relationships in late childhood and early adolescence: Preliminary findings. Australian Educational and Developmental Psychologist, 17, 82-102.

Barrowclough, C., \& Hooley, J. M. (2003). Attributions and expressed emotion: A review. Clinical Psychology Review, 23, 849-880. doi: 10.1016/S0272-7358(03)00075-8

Baucom, D. H., Shoham, V., Mueser, K. T., Daiuto, A. D., \& Stickle, T. R. (1998). Empirically supported couple and family interventions for marital distress and adult mental health problems. Journal of Consulting and Clinical Psychology, 66, 53-88. doi: 10.1037/0022006X.66.1.53

Bieling, P. J., Rowa, K., Antony, M. M., Summerfeldt, L. J., \& Swinson, R. P. (2001). Factor structure of the illness intrusiveness rating scale in patients diagnosed with anxiety disorders. Journal of Psychopathology and Behavioral Assessment, 23, 223-230. doi: 10.1023/A:1012723318964 
Buss, A. H., \& Warren, W. L. (2000). Aggression Questionnaire: Manual. Los Angeles, CA: Western Psychological Services.

Calvocoressi, L., Lewis, B., Harris, M., Trufan, S. J., Goodman, W. K., McDougle, C. J., \& Price, L. H. (1995). Family accommodation in obsessive-compulsive disorder. American Journal of Psychiatry, 152, 441-443.

Calvocoressi, L., Mazure, C. M., Stanislav, K., Skolnick, J., Fisk, D., Vegso, S. J., ... Price, L. H. (1999). Family accommodation of obsessive-compulsive symptoms: Instrument development and assessment of family behavior. Journal of Nervous and Mental Disease, 187, 636-642. doi: 10.1097/00005053-19991000-00008

Calvocoressi, L., Mazure, C. M., Van Noppen, B., \& Price, L. H. (1999). The family accommodation scale for obsessive-compulsive disorder. In G. Steketee, Overcoming obsessive-compulsive disorder: A behavioral and cognitive protocol for the treatment of OCD (pp. 185-200). Oakland, CA: New Harbinger Publications.

Caporino, N. E., Morgan, J., Beckstead, J., Phares, V., Murphy, T. K., \& Storch, E. A. (2012). A structural equation analysis of family accommodation in pediatric obsessive-compulsive disorder. Journal of Abnormal Child Psychology, 40, 133-143. doi: 10.1007/s10802-011$9549-8$

Chambless, D. L., \& Steketee, G. (1999). Expressed emotion and behavior therapy outcome: A prospective study with obsessive-compulsive and agoraphobic outpatients. Journal of Consulting and Clinical Psychology, 67, 658-665. doi: 10.1037/0022-006X.67.5.658

Coles, M. E., Cook, L. M., \& Blake, T. R. (2007). Assessing obsessive compulsive symptoms and cognitions on the internet: Evidence for the comparability of paper and internet 
administration. Behaviour Research and Therapy, 45, 2232-2240. doi:

10.1016/j.brat.2006.12.009

Cole, J. D., \& Kazarian, S. S. (1988). The Level of Expressed Emotion (LEE) scale: A new measure of expressed emotion. Journal of Clinical Psychology, 44, 392-397. doi: 10.1002/1097-4679(198805)44:3<392::AID-JCLP2270440313>3.0.CO;2-3

Damecour, C. L., \& Charron, M. (1998). Hoarding: A symptom, not a syndrome. Journal of Clinical Psychiatry, 41, 179-194. doi: 10.4088/JCP.v59n0512

Derogatis, L. R. (1993). Brief Symptom Inventory: Administration, scoring, and procedures manual. Minneapolis, MN: National Computer Systems.

Devins, G. M. (1994). Illness intrusiveness and the psychosocial impact of lifestyle disruptions in chronic life-threatening disease. Advances in Renal Replacement Therapy, 1, 251-263.

Devins, G. M., Dion, R., Pelletier, L., Shapiro, C. M., Abbey, S., Raiz, L. R., ... Edworthy, S. M. (2001). The structure of lifestyle disruptions in chronic disease: A confirmatory factor analysis of the Illness Intrusiveness Ratings Scale. Medical Care, 39, 1097-1104.

Ferrão, Y. A., Shavitt, R. G., Bedin, N. R., de Mathis, M. E., Lopes, A. C., Fontenelle, L. F., . . . Miguel, E. C. (2006). Clinical features associated to refractory obsessive-compulsive disorder. Journal of Affective Disorders, 94, 199-209. doi: 10.1016/j.jad.2006.04.019

Field, A. (2005). Discovering statistics using SPSS ( $2^{\text {nd }}$ ed.). Thousand Oaks, CA: Sage Publications.

Flessner, C. A., Freeman, J. B., Sapyta, J., Garcia, A., Franklin, M. E., March, J. S., \& Foa, E. (2011). Predictors of parental accommodation in pediatric obsessive-compulsive disorder: Findings from the Pediatric Obsessive-Compulsive Disorder Treatment Study (POTS) trial. Journal of the American Academy of Child and Adolescent Psychiatry, 50, 
716-725. doi: 10.1016/j.jaac.2011.03.019

Foa, E. B., Huppert, J. D., Leiberg, S., Langner, R., Kichic, R., Hajcak, G., \& Salkovskis, P. M. (2004). The Obsessive-Compulsive Inventory: Development and validation of a short version. Psychological Assessment, 10, 206-214. doi: 10.1037/1040-3590.14.4.485

Fredman, S. J., Vorstenbosch, V., Wagner, A. C., Macdonald, A. \& Monson, C. M. (2013). Partner accommodation in posttraumatic stress disorder: Initial testing of the significant others' response to trauma (SORTS) scale. Manuscript submitted for publication.

Frost, R. O., \& Gross, R. C. (1993). The hoarding of possessions. Behaviour Research and Therapy, 31, 367-381. doi: 10.1016/0005-7967(93)90094-13

Frost, R. O., \& Hartl, T. L. (1996). A cognitive-behavioral model of compulsive hoarding. Behaviour Research and Therapy, 34, 341-350. doi: 10.1016/0005-7967(95)00071-2

Frost, R. O., \& Hristova, V. (2011). Assessment of hoarding. Journal of Clinical Psychology: In Session, 67, 456-466. doi: 10.1002/jclp.20790

Frost, R. O., Hristova, V., Steketee, G., \& Tolin, D. F. (in press). Activities of Daily Living Scale in hoarding disorder. Journal of Obsessive-Compulsive and Related Disorders. doi: 10.1016/j.jocrd.2012.12.004

Frost, R. O., Kim, H. J., Morris, C., Bloss, C., Murray-Close, M., \& Steketee, G. (1998). Hoarding, compulsive buying and reasons for saving. Behaviour Research and Therapy, 36, 657-664. doi: 10.1016/S0005-7967(98)00056-4

Frost, R. O., Krause, M. S., \& Steketee, G. (1996). Hoarding and obsessive-compulsive symptoms. Behavior Modification, 20, 116-132. doi: 10.1177/01454455960201006 
Frost, R. O., \& Rasmussen, J. L. (2012). Phenomenology and characteristics of compulsive hoarding. In G. Steketee (Ed.), Oxford handbook of obsessive compulsive and spectrum disorders (pp. 70-88). New York, NY: Oxford University Press.

Frost, R. O., \& Steketee, G. (1998). Hoarding: Clinical aspects and treatment strategies. In M. A. Jenike, L. Baer, and W. E. Minichiello (Eds.), Obsessive-compulsive disorders: Practical management ( $3^{\text {rd }}$ ed., pp. 533-554). St. Louis, MO: Mosby Year Book.

Frost, R. O., \& Steketee, G. (1999). Issues in the treatment of compulsive hoarding. Cognitive and Behavioral Practice, 6, 397-407. doi: 10.1016/S1077-7229(99)80058-3

Frost, R. O., Steketee, G., \& Greene, K. A. I. (2003). Compulsive and behavioral treatment of compulsive hoarding. Brief Treatment and Crisis Intervention, 3, 323-337. doi: 10.1093/brief-treatment/mhg024

Frost, R. O., Steketee, G., \& Grisham, J. (2004). Measurement of compulsive hoarding: Saving Inventory-Revised. Behaviour Research and Therapy, 42, 1163-1182. doi: 10.1016/j.brat.2003.07.006

Frost, R. O., Steketee, G., Tolin, D. F., \& Brown, T. A. (2006, September). Diagnostic issues in compulsive hoarding. Paper presented at the meeting of the European Association of Behavioural and Cognitive Therapies, Paris, France.

Frost, R. O., Steketee, G., Tolin, D. F., \& Renaud, S. (2008). Development and validation of the Clutter Image Rating. Journal of Psychopathology and Behavioral Assessment, 30, 193203. doi: $10.1007 / \mathrm{s} 10862-007-9068-7$

Frost, R. O., Steketee, G., \& Williams, L. (2000). Hoarding: A community health problem. Health and Social Care in the Community, 8, 229-234. 
Frost, R. O., Steketee, G., Williams, L., \& Warren, R. (2000). Mood, personality disorder symptoms and disability in obsessive compulsive hoarders: A comparison with clinical and nonclinical controls. Behaviour Research and Therapy, 38, 1071-1081. doi:

10.1016/S0005-7967(99)00137-0

Frost, R. O., Tolin, D. F., Steketee, G., Fitch, K. E., \& Selbo-Bruns, A. (2009). Excessive acquisition in hoarding. Journal of Anxiety Disorders, 23, 632-639. doi:

10.1016/j.anxdis.2009.01.013

Furby, L. (1978). Possessions: Toward a theory of their meaning and function throughout the life cycle. In P. Bales (Ed.), Life span development and behavior, (Vol. 1, pp. 297-336). New York, NY: Academic Press.

Furr, R. M., \& Bacharach, V. R. (2008). Psychometrics: An introduction. Thousand Oaks, CA: Sage.

Garcia, A. M., Sapvla, J. J., Moore, P. S., Freeman, J. B., Franklin, M. E., March, J. S., \& Foa, E. B. (2010). Predictors and moderators of treatment outcome in the pediatric obsessive compulsive treatment study (POTS I). Journal of the American Academy of Child and Adolescent Psychiatry, 49, 1024-1033. Doi

George, D., \& Mallery, P. (2003). SPSS for windows step by step: A simple guide and reference 11.0 update $\left(4^{\text {th }}\right.$ ed.). Boston, MA: Allyn and Bacon.

Gilliam, C. M., \& Tolin, D. F. (2010). Compulsive hoarding. Bulletin of the Menninger Clinic, 74, 93-121. doi: 10.1521/bumc.2010.74.2.93

Goodman, W. K., Price, L. H., Rasmussen, S. A., Mazure, C., Delgado, P., Heninger, G. R., \& Charney, D. S. (1989). The Yale-Brown Obsessive-Compulsive Scale, II: Validity. Archives of General Psychiatry, 46, 1012-1016. doi: 
10.1001/archpsyc.1989.01810110054008

Goodman, W. K., Price, L. H., Rasmussen, S. A., Mazure, C., Fleischmann, R. L., Hill, C. L., . . . Charney, D. S. (1989). The Yale-Brown Obsessive-Compulsive Scale: Development, use, and reliability. Archives of General Psychiatry, 46, 1006-1011. doi:

10.1001/archpsyc.1989.01810110048007

Gosling, S. D., Vazire, S., Srivastava, S., \& John, O. P. (2004). Should we trust web-based studies? A comparative analysis of six preconceptions about internet questionnaires. American Psychologist, 59, 93-104. doi: 10.1037/0003-066X.59.2.93

Grisham, J. R., Brown, T. A., Liverant, G. I., \& Campbell-Sills, L. (2005). The distinctiveness of compulsive hoarding from obsessive-compulsive disorder. Journal of Anxiety Disorders, 19, 767-779. doi: 10.1016/j.janxdis.2004.09.003

Grisham, J. R., Frost, R. O., Steketee, G., Kim, H. J., \& Hood, S. (2006). Age of onset of compulsive hoarding. Journal of Anxiety Disorders, 20, 675-686. doi:

10.1016/j.janxdis.2005.07.004

Grisham, J. R., Steketee, G., \& Frost, R. O. (2008). Interpersonal problems and emotional intelligence in compulsive hoarding. Depression and Anxiety, 25, 63-71. doi: $10.1002 /$ da. 20327

Havlicek, L. L., \& Peterson, N. L. (1977). Effect of the violation of assumptions upon significance levels of the Pearson r. Psychological Bulletin, 84, 373-377. doi: $10.1037 / 0033-2909.84 .2 .373$

Haynes, S. N., Richard, D. C. S., \& Kubany, E. S. (1995). Content validity in psychological assessment: A functional approach to concepts and methods. Psychological Assessment, 7 , 238-247. doi: 10.1037/1040-3590.7.3.238 
Henry, J. D., \& Crawford, J. R. (2005). The short-form version of the Depression Anxiety Stress Scales (DASS): Construct validity and normative data in a large non-clinical sample. British Journal of Clinical Psychology, 44, 227-239. doi: 10.1348/014466505X29657

Heresco-Levy, U., Brom, D., \& Greenberg, D. (1992). The Patient Rejection Scale in an Israeli sample: Correlations with relapse and physician's assessment. Schizophrenia Research, 8, 81-87. doi: 10.1016/0920-9964(92)90064-C

Hogan, T. P. (2008). Psychological tests and measures. In D. McKay (Ed.), Handbook of research methods in abnormal and clinical psychology (pp. 177-190). Thousand Oaks, CA: SAGE Publications.

Iervolino, A. C., Perroud, N., Fullana, M. A., Guipponi, M., Cherkas, L., Collier, D. A., \& Mataix-Cols, D. (2009). Prevalence and heritability of compulsive hoarding: A twin study. American Journal of Psychiatry, 166, 1156-1161. doi: 10.1176/appi.ajp.2009.08121789

Johnson, V. E. (1986). Intervention: How to help those who don't want help. Minneapolis, MN: Johnson Institute.

Kenny, D. A., Kashy, D. A., \& Cook, W. L. (2006). Dyadic data analysis. New York, NY: Guilford Press.

Kreisman, D. E., Simmens, S. J., \& Joy, V. D. (1979). Rejecting the patient: Preliminary validation of a self-report scale. Schizophrenia Bulletin, 5, 220-222.

Lebowitz, E. R., Panza, K. E., Su, J., \& Bloch, M. H. (2012). Family accommodation in obsessive-compulsive disorder. Expert Review of Neurotherapeutics, 12, 229-238. doi: 10.1586/ern. 11.200 
Lebowitz, E. R., Woolston, J., Bar-Haim, Y., Calvocoressi, L., Dauser, C., Warnick, E., .. .

Leckmann, J. F. (2013). Family accommodation in pediatric anxiety disorders.

Depression and Anxiety, 30, 47-54. doi: 10.1002/da.21998

Lecrubier, Y., Sheehan, D. V., Weiller, E., Amorim, P., Sheehan, K. H., Janavs, J., \& Dunbar, G. C. (1997). The Mini International Neuropsychiatric Interview (MINI). A short diagnostic structured interview: Reliability and validity according to the CIDI. European Psychiatry, 12, 224-231. doi: 10.1016/S0924-9338(97)83296-8

Ledermann, T., Macho, S., \& Kenny, D. A. (2011). Assessing mediation in dyadic data using the actor-partner interdependence model. Structural Equation Modeling, 18, 595-612. doi: $10.1080 / 10705511.2011 .607099$

Livingston-Van Noppen, B., Rasmussen, S. A., Eisen, J., \& McCartney, L. (1990). Family function and treatment in obsessive-compulsive disorder. In M. Jenike, L. Baer, \& W. E. Minichiello (Eds.), Obsessive compulsive disorder: Theory and management $\left(2^{\text {nd }}\right.$ ed., pp. 325-340). Chicago, IL: Year Book.

Lovibond, S. H., \& Lovibond, P. F. (1995). Manual for the Depression Anxiety Stress Scales (2nd ed.). Sydney, Australia: Psychology Foundation.

Mataix-Cols, D., Frost, R. O., Pertusa, A., Clark, L. A., Saxena, S., Leckman, J. F., ... Wilhelm, S. (2010). Hoarding disorder: A new diagnosis for DSM-V? Depression and Anxiety, 27, 556-572. doi: 10.1002/da.20693

McElroy, S. L., Phillips, K. A., \& Keck, P. E., Jr. (1994). Obsessive compulsive spectrum disorder. Journal of Clinical Psychiatry, 55, 33-51.

Merlo, L. J., Lehmkuhl, H D., Geffken, G. R., \& Storch, E. A. (2009). Decreased family accommodation associated with improved therapy outcome in pediatric obsessive- 
compulsive disorder. Journal of Consulting and Clinical Psychology, 77, 355-360. doi: $10.1037 / \mathrm{a} 0012652$

Miller, W. R., Meyers, R. J., \& Tomgan, J. S. (1999). Engaging the unmotivated in treatment for alcohol problems: A comparison of three strategies for intervention through family members. Journal of Consulting and Clinical Psychology, 67, 688-697. doi: 10.1037/0022006X.67.5.688

Mogan, C., Kyrios, M., Schweitzer, I., Yap, K., \& Moulding R. (2012). Phenomenology of hoarding: What is hoarded by individuals with hoarding disorder? Journal of ObsessiveCompulsive and Related Disorders, 1, 306-311. doi: 10.1016/j.jocrd.2012.08.002

Moretz, M. W., \& McKay, D. (2008). Statistical conclusion and construct validity in experimental and quasi-experimental designs. In D. McKay (Ed.), Handbook of research methods in abnormal and clinical psychology (pp. 35-46). Thousand Oaks, CA: Sage Publications.

Myers, K., \& Winters, N. C. (2002). Ten-year review of rating scales. I: Overview of scale functioning, psychometric properties, and selection. Journal of the American Academy of Child and Adolescent Psychiatry, 41, 114-122. doi: 10.1097/00004583-200202000-00004

Peris, T. S., Bergman, R. L., Langley, A., Chang, S., McCracken, T., \& Piacentini, J. (2008). Correlates of accommodation of pediatric obsessive-compulsive disorder: Parent, child, and family characteristics. Journal of the American Academy of Child and Adolescent Psychiatry, 47, 1173-1181. doi: 10.1097/CHI.0b013e3181825a91

Pertusa, A., Frost, R. O., Fullana, M. A., Samuels, J., Steketee, G., Tolin, D. F., . . Mataix-Cols, D. (2010). Refining the diagnostic boundaries of compulsive hoarding: A critical review. Clinical Psychology Review, 30, 371-386. doi: 10.1016/j.cpr.2010.01.007 
Pertusa, A., Fullana, M. A., Singh, S., Alonso, P., Menchon, J. M., \& Mataix-Cols, D. (2008). Compulsive hoarding: OCD symptom, distinct clinical syndrome, or both. American Journal of Psychiatry, 165, 1289-1298. doi: 10.1176/appi.ajp.2008.07111730

Peterson, R. A., \& Reiss, S. (1993). Anxiety Sensitivity Index Revised Test Manual. Worthington, OH: IDS Publishing Corporation.

Piacentini, J., Bergman, L., Keller, M., \& McCracken, J. (2003). Functional impairment in children and adolescents with obsessive-compulsive disorder. Journal of Child and Adolescent Psychopharmacology, 13, 61-69. Doi: 10.1089/104454603322126359

Pierce, G. R., Sarason, I. G., \& Sarason, B. R. (1991). General and relationship-based perceptions of social support: Are two constructs better than one? Journal of Personality and Social Psychology, 61, 1028-1039. doi: 10.1037/0022-3514.61.6.1028

Pierce, G. R., Sarason, I. G., Sarason, B. R., Solky-Butzel, J. A., \& Nagle, L. C. (1997). Assessing the quality of personal relationships. Journal of Social and Personal Relationships, 14, 339-356. doi: 10.1177/0265407597143004

Pinto, A., Van Noppen, B., \& Calvocoressi, L. (in press). Development and preliminary psychometric evaluation of a self-rated version of the Family Accommodation Scale for Obsessive-Compulsive Disorder. Journal of Obsessive-Compulsive and Related Disorders.

Qualtrics Labs (2010). Qualtrics Research Suite (Version no. 16,787) [Computer software]. Provo, UT: Author.

Rachman, S., Elliott, C. M., Shafran, R., \& Radomsky, A. S. (2009). Separating hoarding from OCD. Behaviour Research and Therapy, 47, 520-522. doi: 10.1016/j.brat.2009.02.014 
Ramos-Cerqueira, A. T., Rodrigues Torres, A., Torresan, R. C., Maranhao Negreiros, A. P., \& Nakano Vitorino, C. (2008). Emotional burden in caregivers of patients with obsessivecompulsive disorder. Depression and Anxiety, 25, 1020-1027. doi: 10.1002/da.20431

Renshaw, K. D., Caska, C. M., Rodrigues, C. S., \& Blais, R. K. (2012). The role of family and social relationships in OCD and spectrum conditions. In G. Steketee (Ed.), Oxford Handbook of Obsessive Compulsive and Spectrum Disorders (pp. 168-188). New York, NY: Oxford University Press.

Renshaw, K. D., Chambless, D. L., \& Steketee, G. (2006). The relationship of relatives' attributions to their expressed emotion and to patients' improvement in treatment for anxiety disorders. Behavior Therapy, 37, 159-169. doi: 10.1016/j.beth.2005.11.001

Renshaw, K. D., Steketee, G., \& Chambless, D. L. (2005). Involving family members in the treatment of OCD. Cognitive Behaviour Therapy, 34, 164-175. doi:

$10.1080 / 16506070510043732$

Renshaw, K. D., Steketee, G., Rodrigues, C. S., \& Caska, C. M. (2010). Obsessive-compulsive disorder. In J. G. Beck (Ed.), Interpersonal processes in the anxiety disorders: Implications for understanding psychopathology and treatment (pp. 153-178). Washington, DC: American Psychological Association.

Samuels, J. F., Bienvenu, O. J., Grados, M. A., Cullen, B., Riddle, M. A., Liang, K., . . . Nestadt, G. (2008). Prevalence and correlates of hoarding behavior in a community-based sample. Behaviour Research and Therapy, 46, 836-844. doi: 10.1016/j.brat.2008.04.004

San Francisco Task Force on Compulsive Hoarding (2009). Beyond overwhelmed: The impact of compulsive hoarding and cluttering in San Francisco and recommendations to reduce 
negative impacts and improve care. San Francisco, CA: San Francisco Task Force on Compulsive Hoarding.

Saxena, S., \& Maidment, K. M. (2004). Treatment of compulsive hoarding. Journal of Clinical Psychology, 60, 1143-1154. doi: 10.1002/jclp.20079

Sheehan, D. V. (1983). The anxiety disease. New York, NY: Scribner.

Smith, J. E., \& Meyers, R. J. (2004). Motivating substance abusers to enter treatment: Working with family members. New York, NY: Guilford Press.

Steketee, G., Ayers, C., Umbach, A., Tolin, D. F., \& Frost, R. O. (2013). Family Response to Hoarding: Assessment and features in an internet sample. Submitted for publication.

Steketee, G., \& Frost, R. O. (2003). Compulsive hoarding: Current status of the research. Clinical Psychology Review, 23, 905-927. doi: 10.1016/j.cpr.2003.08.002

Steketee, G., \& Frost, R. O. (2007). Compulsive hoarding and acquiring: Therapist guide. New York, NY: Oxford University Press.

Steketee, G., Frost, R. O., \& Kim, H. J. (2001). Hoarding by elderly people. Health and Social Work, 26, 176-184.

Steketee, G., Frost, R. O., \& Kyrios, M. (2003). Cognitive aspects of compulsive hoarding. Cognitive Therapy and Research, 27, 463-479. doi: 10.1023/A:1025428631552

Steketee, G., \& Van Noppen, B. (2003). Family approaches to treatment of obsessive compulsive disorder. Journal of Family Psychotherapy, 14, 55-71. doi: 10.1300/J085v14n04_06

Stewart, S., Beresin, C., Haddad, S., Stack, D. E., Fama, J., \& Jenike, M. (2008). Predictors of family accommodation in obsessive-compulsive disorder. Annals of Clinical Psychiatry, 20, 65-70. doi: $10.1080 / 10401230802017043$ 
Storch, E. A., Geffken, G. R., Merlo, L. J., Jacob, M. L., Murphy, T. K., Goodman, W. K., . . . Grabill, K. (2007). Family accommodation in pediatric obsessive-compulsive disorder. Journal of Clinical Child and Adolescent Psychology, 36, 207-216.

Storch, E. A., Geffken, G. R., Merlo, L. J., Mann, G., Duke, D., Munson, M., . . Goodman, W. K. (2007). Family-based cognitive-behavioral therapy for pediatric obsessive-compulsive disorder: Comparison of intensive and weekly approaches. Journal of American Academy of Child and Adolescent Psychiatry, 46, 469-478. doi: 10.1097/chi.0b013e31803062e7

Storch, E. A., Lack, C. W., Merlo, L. J., Geffken, G. R., Jacob, M. L., Murphy, T. K., \& Goodman, W. K. (2007). Clinical features of children and adolescents with obsessivecompulsive disorder and hoarding symptoms. Comprehensive Psychiatry, 48, 313-318. doi: 10.1016/j.comppsych.2007.03.001

Tabachnick, B. G., \& Fidell, L. S. (2007). Using multivariate statistics $\left(5^{\text {th }}\right.$ ed.). Boston, MA: Pearson.

Tolin, D. F., Fitch, K. E., Frost, R. O., \& Steketee, G. (2010). Family informants' perceptions of insight in compulsive hoarding. Cognitive Therapy and Research, 34, 69-81. doi: $10.1007 / \mathrm{s} 10608-008-9217-7$

Tolin, D. F., Frost, R. O., \& Steketee, G. (2010). A brief interview for assessing compulsive hoarding: The Hoarding Rating Scale - Interview. Psychiatry Research, 178, 147-152. doi: 10.1016/j.psychres.2009.05.001

Tolin, D. F., Frost, R. O., Steketee, G., \& Fitch, K. E. (2008). Family burden of compulsive hoarding: Results of an internet survey. Behaviour Research and Therapy, 46, 334-344. doi: 10.1016/j.brat.2007.12.008 
Tolin, D. F., Frost, R. O., Steketee, G., Gray, K. D., \& Fitch, K. E. (2008). The economic and social burden of compulsive hoarding. Psychiatry Research, 160, 200-211. doi: 10.1016/j.psychres.2007.08.008

Tolin, D. F., Meunier, S A., Frost, R. O., \& Steketee, G. (2010). Course of compulsive hoarding and its relationship to life events. Depression and Anxiety, 27, 829-838. doi: 10.1002/da.20684

Van Noppen, B., \& Steketee, G. (2009). Testing a conceptual model of patient and family predictors of obsessive compulsive disorder (OCD) symptoms. Behaviour Research and Therapy, 47, 18-25. doi: 10.1016/j.brat.2008.10.005

Weiner, B. (1986). An attributional theory of motivation and emotion. New York, NY: SpringerVerlag.

Wilbram, M., Kellett, S., \& Beail, N. (2008). Compulsive hoarding: A qualitative investigation of partner and carer perspectives. British Journal of Clinical Psychology, 47, 59-73. doi: $10.1348 / 014466507 X 240740$ 